\title{
Subterranean biota of the European part of Russia: A review
}

\section{Sergei I. Golovatch ${ }^{1}$, Dmitry M. Palatov ${ }^{2}$, Ilya S. Turbanov ${ }^{3,4}$, Vladimir A. Kniss ${ }^{5}$, Suren Gazaryan ${ }^{6}$, Vladimir P. Snit'ko, †Vasile Decu ${ }^{8}$, Christian Juberthie ${ }^{9}$, George Nazareanu ${ }^{10,11}$}

\author{
${ }^{1}$ Institute for Problems of Ecology and Evolution, Russian Academy of Sciences, Leninsky pr., 33, \\ Moscow, 119071 Russia.E-mail: sgolovatch@yandex.ru \\ ${ }^{2}$ Department of Hydrobiology, Biological Faculty, Moscow State University, Leninskie Gory 1/12, \\ Moscow, 119234 Russia; E-mail: triops@yandex.ru \\ ${ }^{3}$ Papanin Institute of the Biology of Inland Waters, Russian Academy of Sciences, Borok, Nekouzskiy \\ District, Yaroslavl Region, 152742 Russia.E-mail: turba13@mail.ru \\ ${ }^{4}$ Cherepovets State University, Lunacharskogo pr., 5, Cherepovets, Vologda Region, 162600 \\ Russia. \\ ${ }^{5}$ Bashkirian University, 32 rue Frunze, Ufa, 450074 Russia. \\ ${ }^{6}$ UNEP/EUROBATS Secretariat, Platz der Vereinten Nationen 1, D-53113 Bonn, Germany. E-mail: \\ s.gazaryan@gmail.com \\ 7 Il'men State Nature Reserve, Chelyabinsk Region, Miass, 456317 Russia. E-mail: \\ snitko@ilmeny.ac.ru \\ ${ }^{8}$ Dept. of Biospeleology and Karst Edaphobiology, "Emil Racovitza" Institute of Speleology, \\ Romanian Academy, Calea 13 Septembrie, 13, Bucharest, 050731 Romania. \\ ${ }^{9}$ Encyclopédie Biospéologique, Edition. 1 Impasse Saint-Jacques, F-09190 Saint-Lizier, France. E- \\ mail: christianjuberthie@wanadoo.fr \\ 10 "Grigore Antipa" Nationa Museum of Natural History, Sos. Kiseleff, 1, 011341 Bucharest, \\ Romania. \\ ${ }^{11}$ Karin's Kids Academy - Nature School, Sos. Bucuresti-Ploiesti, 182A, 013697 Bucharest, \\ Romania.E-mail: george.nazareanu@karinkids.ro
}

ABSTRACT. The endogean flora and fauna of European Russia, including those of the northwestern Caucasus, but excluding those of Crimea, presently comprise at least 389 species or subspecies (some still unidentified) from 229 genera (a few still unidentified), 150 families, 75 orders, 48 classes and 25 phyla, among which 96 species or subspecies represent presumed stygo- or troglobionts. The taxonomically most diverse phylum is Arthropoda which encompasses the bulk of the fauna, in particular due to crustaceans, beetles and collembolans. Whereas the troglofauna of northern Russia is completely devoid of such strongly cave-adapted animals, in the Urals, Cis-Urals and central Russia there are a few, but the greatest diversity of hypogean species in the region is observed in the northwestern Caucasus, in particular its maritime western part, as an "Ice Age" refugium of nemoral biota rich in karst.

How to cite this article: Golovatch S.I., Palatov D.M., Turbanov I.S., Kniss V.A., Gazaryan S., Snit'ko V.P., Decu V., Juberthie Ch., Nazareanu G. 2018. Subterranean biota of the European part of Russia: A review // Invert. Zool. Vol.15. No.2. P.153-213. doi: 10.15298/ invertzool.15.2.01

KEY WORDS: Eastern Europe, Urals, Cis-Urals, northern Caucasus, endogean invertebrates, bats, stygophiles, stygobionts, troglophiles, troglobionts. 


\title{
Подземная биота европейской части России: Обзор
}

\section{С.И. Головач', Д.М. Палатов 2 , И.С. Турбанов ${ }^{3,4}$ В.А. Книсс ${ }^{5}$, Сурен Газарян ${ }^{6}$, В.П. Снитько ${ }^{7}$ †Василе Деку Кристиан Жюберти ${ }^{9}$, Георге Назаряну ${ }^{10,11}$}

\author{
${ }^{1}$ Институт проблем экологии и эволюичии РАН, Ленинский проспект, 33, Москва, 119071 \\ Россия. \\ ${ }^{2}$ Кафедра гидробиологии, Биологический факультет МГУ, Ленинские Горы, 1/12, Москва, \\ 119234 Россия \\ ${ }^{3}$ Институт биологии внутренних вод РАН, пос. Борок, Ярославская обл., 152742 Россия. \\ ${ }^{4}$ Череповецкий государственный университет, проспект Луначарского, 5, Вологодская обл., \\ 162600 Россия. \\ ${ }^{3}$ Башкирский государственный университет, ул. Фрунзе, 32, Уфа, 450074 Россия. \\ ${ }^{6}$ Секретариат UNEP/EUROBATS, Бонн, Германия. \\ ${ }^{7}$ Ильменский государственный заповедник, г. Миасс, Челябинская обл., 456317 Россия. \\ ${ }^{8}$ Институт спеологии, Румынская академия наук, Бухарест, Румыния. \\ ${ }^{9}$ Издательство Биоспелеологической эничиклопедии, Сен-Лизье, Франция. \\ ${ }^{10}$ Музей естественной истории, Бухарест, Румыния. \\ ${ }^{11}$ Детская академия Карины, Бухарест, Румыния.
}

РЕЗЮМЕ. Подземные флора и фауна европейской части России, включая таковые Северо-Западного Кавказа, но исключая таковые Крыма, в настоящее время насчитывают по меньшей мере 389 вида и подвида (некоторые пока не определены) из 229 родов (малая часть пока не определена), 150 семейств, 75 отрядов, 48 классов и 25 типов, среди которых 96 видов и подвидов - предположительно стиго- и троглобионты. Таксономически самый разнообразный тип - это Arthropoda, который включает большинство фауны, особенно за счет ракообразных, жуков и коллембол. В то время, как троглофауна северной части России совершенно лишена сильно адаптированных в жизни в пещерах и подземных водах животных, на Урале, в Предуралье и центре России таковых немного, а наибольшее разнообразие гипогейных видов в регионе наблюдается на Северо-Западном Кавказе, прежде всего, в его приморской западной части, как богатого карстом «ледникового» рефугиума неморальной биоты. Как цитировать эту статью: Golovatch S.I., Palatov D.M., Turbanov I.S., Kniss V.A., Gazaryan S., Snit'ko V.P., Decu V., Juberthie Ch., Nazareanu G. 2018. Subterranean biota of the European part of Russia: A review // Invert. Zool. Vol.15. No.2. P.153-213. doi: 10.15298/invertzool.15.2.01

КЛЮЧЕВЫЕ СЛОВА: Восточная Европа, Урал, Предуралье, Северный Кавказ, подземные беспозвоночные, летучие мыши, стигофилы, стигобионты, троглофилы, троглобионты.

\section{Introduction}

The present paper is the result of a compromise. It was initiated by one of us (CJ) to become a chapter entitled "RUSSIA (Russian Federation: European part)" in a forthcoming volume of "ENCYCLOPAEDIA BIOSPEOLOGICA". The chapter was duly prepared, but then remained shelved for more than a year waiting for some other contributions to be com- pleted for the same volume. However, because of uncertainty and an apparent delay in publication, let alone an unexpected abortion of the part pertaining to mammals in our prepared draft, we have finally decided to publish this material elsewhere in a reputed journal and before it is outdated. This decision actually follows that of Juberthie et al. (2016) who published their chapter on the cave faunas of Siberia and the Russian Far East as a journal paper. The same concerns 
the chapter on the Mexican hypogean fauna by Palacios-Vargas et al. (2017). As regards the present contribution that covers European Russia, a separate chapter will be devoted to the troglofauna of Crimea.

\section{General description of the area}

The Russian Federation is the largest country in the world $\left(17,075,000 \mathrm{~km}^{2}\right)$, spanning $10,000 \mathrm{~km}$ from the Baltic Sea in the West to the Pacific (the Sea of Japan, the Okhotsk Sea, the Bering Sea) in the East. The Ural Mountain Range represents the traditional border between European Russia and Siberia (Asian Russia), lying between Europe and Asia.

The European part consists of large plains; the southern part of European Russia consists of the northern and western Caucasus, including a part of Transcaucasia stretched along the Black Sea coast until Sochi and the Psou River, both bordering on Abkhazia. Central Siberia is mostly a large plateau; southern Siberia bordering on Mongolia and China, as well as the Far East as far as the Sea of Okhotsk and the Sea of Japan are mountainous regions.

Climate. Temperate and continental to the West, with marine influence of the Baltic Sea and the Sea of Japan; cold and subpolar to the North, nearly subtropical in the Sochi region along the Black Sea. The latitude, the distance to the ocean and the localization of mountains account for climate continentality; the climate of Siberia is characterized by mostly very severe and harsh winters.

Vegetation. From north to south, the pattern of nature zonation is formed by a marked succession of the following biomes: polar deserts, tundras, taiga (= boreal, mostly coniferous forest), broadleaved forest, forested steppes, steppes (= grasslands), semi-deserts and deserts.

\section{History}

\section{1. Karst}

In Russia, the first descriptions of karst forms and caves were made as early as the 18th century by several academic expeditions, fol- lowing the conquest of the huge territories of the Urals, Siberia and, later, the Far East. The first detailed field manual on caves was published by J. Gmelin as a result of the Great North Expedition carried out between 1733 and 1743 .

At the beginning of the 19th century, V. Severgin published a review of the caves in the Russian Empire.

In 1887, Yuri Listov published a field manual on cave studies with notes on their geology, morphology, hydro-geology, speleogenesis, sediments and microclimate.

In 1900, Alexander Kruber published an important book, "About karst phenomena in Russia". The role of Kruber in Russia is somewhat comparable to that of E.A. Martel in Western Europe. Martel visited Russia in 1903.

From the beginning of the $20^{\text {th }}$ century, the number of regional karst cave studies continued to grow in all of the main regions of Russia.

The revolution of 1917 interrupted karst and cave research in the country, but it resumed in the USSR.

The first Karst Conference in the USSR was held in 1933 at Kizel, Perm Region. Since 1947, the Perm State University sponsored all-Union conferences on karst and played important roles in the coordination of karst speleological investigations.

Regional thematic conferences on karst and speleology were organized by the Karst Commission, for instance on Applied Karstology, Russian Plain karst, Bashkirian karst, Primorskyi Krai karst, Caves, engineering karstology etc.

According to A. Klimchouk (2003c), modern speleology in the USSR was born in 1958, when the Academy of Sciences created the Interdepartmental Commission on Geological and Geographical Karst Investigations.

General studies on Russian karst were published by Klimchouk (2003b), Maksimovitch (1962, 1963), Maksimovitch and Kostarev (1973), Gorbunova et al. (1992) and many others. Special studies were published on gypsum karsts of the Russian Eastern-European Plain and the Pre-Ural region by Andrejchuk (1996), and Andrejchuk and Klimchouk (1996). 
Until 1958, only about 500 caves and shafts were reported in the Soviet Union as a whole. From 1958 to 1962 , cave-exploring groups were formed, with as many as 3,800 caves discovered in the USSR (Russia, Ukraine, Caucasus) (Dublyanskiy, Ilyukhin, 1982; Kiselyov, Klimchouk, 1991).

\section{2. Cave fauna}

The first mention concerning Russia's cave fauna belonged to P. Pallas, a famous academician and traveler, who, in 1811, reported the presence of bat colonies in caves of Crimea and the Urals. Then around the middle of the $19^{\text {th }}$ century, as industrial development required resources, geological research was conducted in the eastern regions such as the Urals, the Altais and Transbaikalia, as well as in the Caucasus and other regions of the Russian Empire.

Victor von Motschoulsky, a famous entomologist, first visited several Caucasian caves in 1850, then some caves in Crimea.

In 1889, G. Jakobson and R. Schmidt sampled fauna in the Murandimovskaya and Kapova caves in the southern Urals and recorded the dipteran Blepharoptera modesta, Culicidae larvae and adults, Collembola and Aranei. From among the species collected, Skorikow (1899) described a new species of springtail, Tomocerus baschkiricus.

Understanding the importance of cave studies in resolving biogeographical problems and those linked to evolutionary theory, G. Kozhevnikov, professor of the Moscow Universty, created in the early $20^{\text {th }}$ century, inside the Zoological Museum, a "dark room" for experimenting on the impact of darkness upon Crustacea and fishes (Ognev, 1910; Kapterev, 1910, 1912).

Nevertheless, the true beginning of biospeleological research in Russia is dated 1912, when A. Shugurov generalized the then available data, relatively few, on the cave fauna of the Caucasus and Crimea (Shugurov, 1912).

Biospeleological investigations were resumed only in 1926-1927, already in the USSR, with explorations of some caves in the Urals and the description by E. Borutzky in 1928 of the first stygobiont, the amphipod Crangonyx chlebnikovi from the central Urals. In 1929, Borutz- ky joined the first biospeleological survey of two karstic caves in the Kutaisi region of Georgia, Transcaucasia, and published the first data about the cave fauna of Transcaucasia (Borutzky, 1930, 1934).

In 1927 and 1930, A.N. Derzhavin published two papers containing descriptions of new cave amphipods.

At the end of 1930, K. Verhoeff described a diplopod, Leucogeorgia longipes, from a cave near Kutaisi, Georgia (Verhoeff, 1930).

Since 1935, Ya. Birstein undertook 6 trips in the Caucasus and explored 39 caves: 31 along the Black Sea coast, 4 in the Imeretian region of Georgia and 3 in Crimea.

In 1940, Birstein and Lopashov published a synthesis of the cave fauna of the USSR as revealed in 1935-1940. That paper also marked the onset of the series "Biospelogica Sovietica" (Birstein, Lopashov, 1940). At the same time, I. Lapshov (1940) reviewed the pseudoscorpion cave fauna of Transcaucasia.

In 1950, Birstein generalized and updated all available information on the cave fauna of western Transcaucasia (Birstein, 1950).

In 1950, E. Borutzky described a stygobiont copepod, Speocyclops lussianus, from Ciscaucasia (Borutzky, 1950).

From 1950 to 1970, Birstein alone or together with Ljovuschkin, Borutzky, Levanidov or Lopashov, published mostly on aquatic cave fauna. This work culminated in a milestone synthesis of biospeleological research in the USSR (Birstein, Ljovuschkin, 1967a). During the same period, Ljovuschkin alone or with coauthors described a number of cave beetles, crustaceans and arachnids, Borutzky described numerous new species of cave copepods and isopods, Ya. Starobogatov and P. Matiokin worked on molluscs, N. Zalesskaja on lithobiomorphs, I. Malevich on earthworms, etc.

Since the 1970's, V. Kniss has studied the fauna of caves and springs, mostly in the Urals and in Siberia. In 2001, he published a summarizing monograph, "Cave fauna of Russia and adjacent regions". From 1984 to 2006, V. Kniss published mostly on springtail cave fauna (Kniss, 1984a, b, 1985, 1989, 1991, 2001, 2004, 2006). 
It is Birstein who may be credited as the pioneer of biospeleology in the USSR, with several of his colleagues or direct students having provided the foundations for our present knowledge of the endogean faunas of the exUSSR countries.

The cave invertebrate fauna of the former Soviet Union, which represents the bulk of endogean biodiversity, has very recently been revised and summarized (Turbanov et al., 2016a, b, c). Members of 17 phyla, 38 classes, 90 orders and 278 families of invertebrates which contain at least 308 species or subspecies of mostly presumed stygo- or troglobionts and no fewer than 735 species or subspecies of stygoor troglophiles are currently known to populate the caves and subterranean waters of Russia and other countries of the former USSR. The main evolutionary burst in the endogea, including the MSS (milieu souterrain superficiel, or mesovoid shallow stratum), is due to arthropods, primarily crustaceans, collembolans and beetles. The major centres of taxonomic diversity among stygo- and troglobionts thereby remain the Caucasus (181, or almost 59\%) and Crimea (44, or over $14 \%$ ), both montane "glacial" refugia of nemoral biota rich in karst, whereas the contributions of the other major regions, sometimes strongly karstified as well, are considerably smaller, gradually decreasing towards Central Asia (35, or more than $11 \%$ ), the Far East (33, or nearly $11 \%$ ), the Ukrainian Carpathians together with Podolia (12, or almost $4 \%$ ), the Russian Plain (7, or over 2\%), the Urals and Cisuralia (7, also more than 2\%) and Siberia (5, or $1.6 \%)$. The problem of inventorying is still among the most topical in assessing the stygoand troglofaunas of the territories in question.

Although occasional findings of bats in caves were reported by many Soviet zoologists (see Kuzyakin (1950)) and the importance of these sites was obvious, Petr Petrovich Strelkov was the first Russian bat researcher who focused on underground sites and conducted an extensive survey of those caves and artificial underground roosts that were known at that time in European Russia (Strelkov, 1958), establishing a tradition for winter bat monitoring. Following his pilot survey, underground sites had become a key element of all regional studies of bat fauna. They gained a momentum after the first allUSSR bat research conference that was held in Leningrad (now Saint Petersburg) in 1974 and where the commission on bats was organized under the auspices of the Soviet Theriological Society. The conference had become a regular event with a number of attendees from the former USSR who reported new findings in a compendium of proceedings (1974, 1980, 1982, 1988, 1990). Altogether, more than 120 papers and short communications on the distribution of bats in the territory of the former USSR had been published by 1990 (Strelkov, 1990); a great deal of them containing data collected in caves and underground sites.

After the dissolution of the Soviet Union, the coordination of bat studies in Russia was taken over by the Russian Bat Research Group that was established at Seventh All-Russian Bat Research Conference (13-16 April 1999). The Group issues its own periodical, Plecotus et al., and maintains a website at http://zmmu.msu.ru/bats

Apart from papers and proceedings, several comprehensive regional bat surveys have recently been conducted and subsequently published as dissertations and monographs. With respect to the cave bat fauna, it is worth mentioning a study on bats of the Western Caucasus (Gazaryan, 2002) and those for the central and southern Ural regions (Orlov, 2000; Snit'ko, 2004). The results of the latter two studies largely constitute the monograph "Bats of the Urals" (Bol'shakov et al., 2005) that was later amended by several new publications of Vladimir Snit'ko, who explored summer and winter bat assemblages in the caves of Bashkiria and the Orenburg and Chelyabinsk regions. In 1999, S. Gazaryan and A. Ostapenko published the first list of 49 caves which are important for bats in the Russian Caucasus and should be legally and physically protected; the paper included recommendations to cavers concerning bat-friendly conduct and the timing of visits to these caves. In a series of further publications $\mathrm{S}$. Gazaryan appended this list with many new important sites which he had discovered during his field trips in 1999-2012. 
Since 1975, several groups have become particularly well studied in the Russian Caucasus, such as cave diplopods (Golovatch, 1975, 1978, 1983, 1984-1985, 1990, 2011; Golovatch, Enghoff, 1990; Golovatch, Chumachenko, 2013; Golovatch et al., 2016; Antić, Makarov, 2016; Antić et al., 2018); cave carabids (Belousov, 1989, 1999, 1998; Belousov, Zamotajlov, 1999, 1997; Belousov, Koval, 2009, 2011) and a few other higher taxa, mostly with descriptions of numerous new species.

A highly detailed survey of the invertebrate cave fauna of the former Soviet Union, including Russia, and a complete bibliography was published recently by Turbanov et al. (2016a, b, c). The present paper is not only restricted to the European part of Russia, including the northern Caucasus, but excluding Crimea (the latter is to be treated separately), but it incorporates all previously unpublished data contained in the PhD Thesis of S.I. Ljovuschkin (1966). It is also slightly updated using relevant information, both new and missed old, but in addition it covers bats as a highly important component of cave fauna. Besides this, a geological background is provided here.

No zoogeographical analysis is undertaken here since such is available and still quite valid as regards the entire subterranean fauna of the former USSR (Kniss, 2004). In addition, European Russia, especially the northern Caucasus with its particularly rich endogean fauna, is an artificial and heterogeneous region from a biogeographical point of view.

\section{Karst and caves}

The Precambrian craton occupies most of the European part of Russia. Karst is mainly developed in intrastratal settings in limestones, dolomites, chalk, gypsum and salt of different ages. Most of the caves are in gypsum.

The karstic caves were developed in rocks from a wide range of geological age: Archaean, Proterozoic, Palaeozoic, Triassic, Jurassic, in limestones, dolomites, mixted carbonate rocks, carbonate conglomerates, and gypsum. Pseudokarstic caves are known in sandstones.

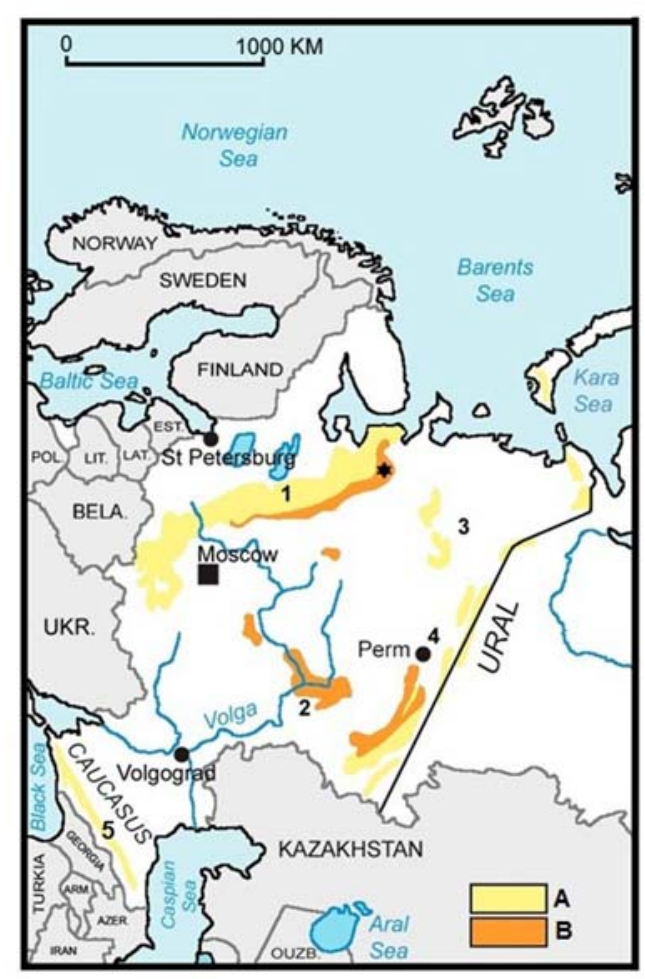

Fig. 1. Karsts of European Russia. A — Carbonated rocks. B - Carbonated and Gypsum rocks. Numbers: 1 - Valdai-Kuloisk: limestone and gypsum karsts; 2 - Volga-Kama; 3 - Timan Plateau; 4 - Urals; 5 - Great and Lesser Caucasus. * Pinega gypsum caves. (After Pulina (2005), modified; drawing by B. Juberthie and G. Nazareanu).

Рис. 1. Карсты европейской части России. Акарбонатные породы. В - карбонатные и гипсовые породы. Номера: 1 - Валдай-Кулой: известняковые и гипсовые карсты; 2 - ВолгоКамские; 3 - Тиманское плато; 4 - Урал; 5 Большой и Малай Кавказ. * Пинежские гипсовые пещеры. (По Pulina (2005) с изменениями; рисунок В. Juberthie и G. Nazareanu).

A. Chikishev $(1966,1973,1978,1984)$ distinguished 12 speleological areas in the former USSR. To date, based on morpho-structural criteria, we distinguish 5 karstic-speleologic regions in the European part of Russia: ValdaiKuloisk, Volga-Kama, Timan Plateau, Russian Great Caucasus, Ural (Fig. 1). 


\section{The Valdai-Kuloisk speleological} region. The northern part of the Russian Plain

Located in the northwestern part of European Russia, between Moscow and St. Petersburg, this region is characterized by a very large, but fragmented limestone and gypsum karst strip, $50-100 \mathrm{~km}$ broad, $1000 \mathrm{~km}$ from south to north near the Baltic Sea.

These northern karsts of European Russia are subjected to hard climate below the arctic anticyclone, with oceanic traits. The rugged karst landscape is mostly overgrown with taiga forest of birch and spruce.

The northwestern Russian area includes some of the largest gypsum karst regions in Europe. The gypsum-anhydrate sequence, often intercalated with limestones and dolomites, is usually 40-60 m thick and lies at shallow depths. Near $40 \%$ of the caves are active, partly phreatic, air and water temperature often being near $0{ }^{\circ} \mathrm{C}$.
More than 360 gypsum caves are known, their total length is over $100 \mathrm{~km}$. The longest caves occur in gypsum karst and include the Kulogorskaya-1-2-Troya (14 km), Konstitutsionaya $(5.8 \mathrm{~km})$ and Olimpiyskaya $(5.5 \mathrm{~km})$ caves (Malkov, Gurkalo, 1999; Klimchouk, 2003b).

They include Pinega gypsum caves in a Permian gypsum outcrop west of the Urals, around the small town of Pinega, east of Arkhangelsk, just outside the Arctic zone of permafrost, in the main bed of evaporites (gypsum and anhydrite). The ground never freezes to depths of more than a few meters, and the cave streams continue flowing throughout the winter. Over $50 \mathrm{~km}$ of passages have been mapped in the group of Pinega caves, among them 22 caves are longer than $1 \mathrm{~km}$ each. Three of the most important caves are the Olimpiyskaya, Lomonosovskaya and Muzeynaya (= Muzeyskaya) caves, in the Iron Gates Sanctuary and the Pinega (=Pinezhskiy) Nature Reserve (Fig. 2) (Waltham, 1994, 2003; Waltham, Cooper, 1998).

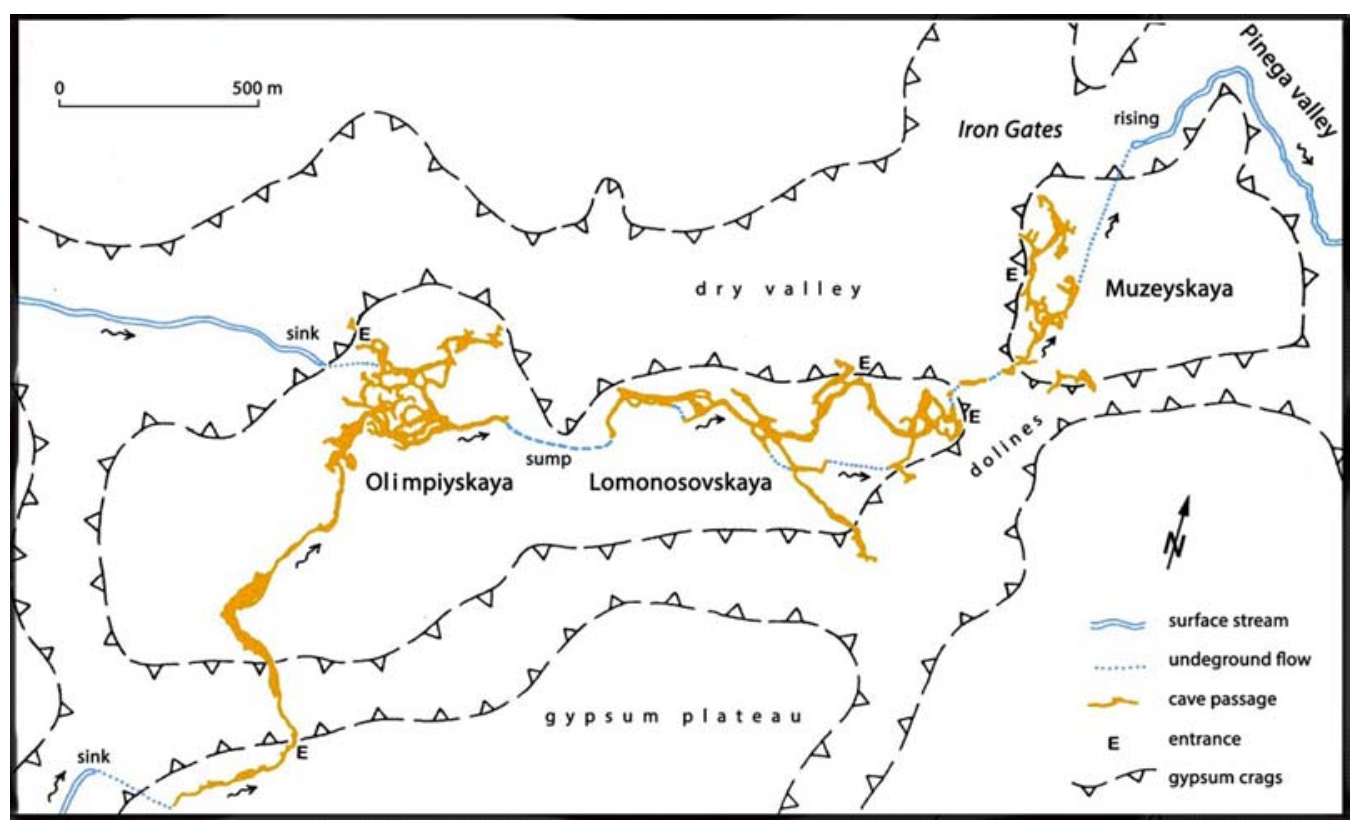

Fig. 2. Pinega Gypsum caves. Maps of three caves located at the Iron Gates (near the Pinezhskiy Nature Reserve). After surveys by "Arkhangelsk Geologiya” and Waltham (2003); coloured by B. Juberthie. Рис. 2. Пинежские гипсовые пешеры. Карта трех пещер, расположенных у Железных Ворот (рядом с Пинежским заповедником). По обследованиям «Архангельской геологии» и Waltham (2003); раскрасил В. Juberthie). 
Many caves are truncated in the valley walls. A large proportion of caves have dendritic passages that carry or carried subterranean streams.

The Simfoniya (3.2 km long) and Golubinskaya (1.6 km long) caves are both maze caves without stream, located at higher levels in the gypsum plateau. They are comparable in appearance to those in the gypsum of Ukraine.

Below some large entrances cave glaciers are created; the oldest ice has been dated to 3000 years ago (Andrejchuk, Klimchouk, 1996; Malkov et al., 1986, 1988).

\section{Volga-Kama (the Volgo-Kamsky region)}

This region lies between the Volga and the Urals. Karst is developed in Permian limestones, dolomites and sulfates, and is in Carboniferous and Devonian carbonate rocks.

In the middle Volga and Kama basins, four relatively large zones of limestone and gypsum karst are recorded. They consist in gypsum beds, thickness $10-45 \mathrm{~m}$, interstrated to limestone and dolomite of lower Permian age. There are springs, commonly discharging sulfate-rich water from deep-seated or semi-confined aquifers (Stupishin, 1965; Andrejchuk, Klimchouk, 1996).

The caves are not numerous, their length modest (as far as $100 \mathrm{~m}$ ), and they still remain poorly-studied in a biospeological aspect (Klimchouk, 2003b).

\section{The Timanskiy Mountain Ridge}

Within the taiga, or the boreal forest belt, from the northern Urals to the Arctic Ocean, lies the Timanskiy Mountain Ridge which contains Devonian and lower Permian gypsum karsts intercalated in dolomites and limestones (Torsuev, 1975). Boreholes and mines have intercepted numerous cavities yielding sulfate-rich water.

\section{The Urals (Fig. 3)}

Karst, gypsum karst, and caves were explored, described and studied by Andrejchuk (1996), Andrejchuk and Klimchouk (1996),

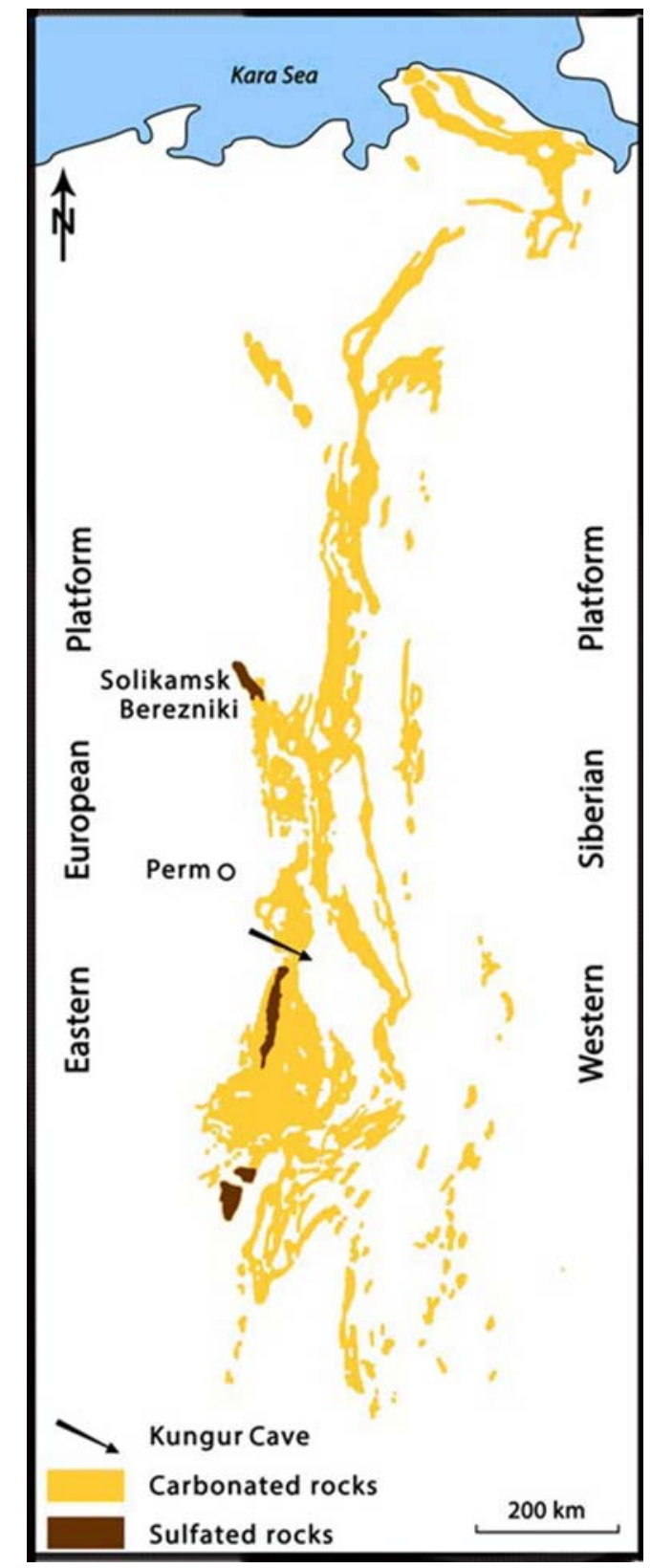

Fig. 3. Karsts of the Urals: carbonated and sulfated rocks.

After Salomon and Pulina (2005), modified by G. Nazareanu.

Рис. 3. Карсты Урала: карбонатные и сульфатные породы.

По Salomon and Pulina (2005), изменения G. Nazareanu. 
Chikishev (1973), Dublyanskiy and Ilyukhin (1982), Gorbunova (1965, 1977, 1979), Gorbunova et al. (1992); Kiselyov and Klimchouk (1991), Maksimovitch (1963), Maksimovitch and Gorbunova (1958), Maksimovitch and Kostarev (1973) etc.

The Ural Mountain Ridge covers 2000 km from north to south and represents the traditional boundary between Europe and Asia. It is a Hercynian folded and eroded system largely covered by woodlands. Approximately 1,800 caves are recorded in gypsum and anhydrite rocks of Permian age, as well as in Devonian and Carboniferous limestones and dolomites.

The largest caves are located in the middle and southern Urals: the Sumgan-Kutuk (9.9 $\mathrm{km})$, Divya $(9.1 \mathrm{~km})$ and Kinderlinskaya (7.9 $\mathrm{km})$ caves, as well as the Kungurskaya Cave $(5.4 \mathrm{~km})$ show in the Perm Region. The Shulgan-Tash Cave, $2.6 \mathrm{~km}$ of passages, is famous for its Palaeolithic paintings. The most important karst springs are located in the western Urals: the Goluboe Ozero springs and the Krasnyi Klyuch Spring.

In the northern Urals, near the Polar Circle (Paykhoy and arctic regions), karst was developed under and inside the permafrost (Klimchouk, 2003b).

Gypsum karst in the Urals is widespread, mainly present along the Urals' western periphery (Maksimovitch, Gorbunova, 1958; Gorbunova, 1965, 1977, 1979; Maksimovitch, Kostarev, 1973; Lysenin, 1981; Makukhin, Molodkin, 1988; Gorbunova et al., 1992; Andrejchuk, 1996).

These extend from the Arctic islands of Novaya Zemlya in the North as far as the hot deserts of the northern Caspian region in the South.

In the central Urals, several hundred publications concern gypsum karsts. About 200 caves have been explored in the gypsum: the largest are the Kungurskaya (= Kungur Ice Cave) (5.6 $\mathrm{km})$ and the Zuyatskaya (1,410 m long) caves. Karstic springs are numerous.

Studies on cave fauna were mainly performed in caves of the western macro-slope of the central Urals.

\section{The Russian Great Caucasus}

The Great Caucasus, or Caucasus Major (Bolshoy Kavkaz), is the highest mountain ridge in European Russia, where many mountain peaks are 3,000-5,000 $\mathrm{m}$ in elevation (Mt Elbrus, volcanic peak, 6,642 $\mathrm{m}$ a.s.1.), spanning 1,250 $\mathrm{km}$ from the Black Sea to the Caspian Sea.

Only the northern macro-slope (Ciscaucasia, or Circassia) and the westernmost part of the Caucasus, from Novorossiysk as far as Sochi and the Psou River, neighbouring to Abkhazia, along the Black Sea coast, is Russian.

On the northern macro-slope, the karsts were developed in monoclinal and folded structures, mostly in Jurassic and lower Cretaceous limestones, dolomites, and gypsum. Alpine karst and deep caves are present in the northern slope and Russian western region. As of 2003, the Gorlo Barloga Cave was the deepest (-900 m), located in the Zagedan Massif in Devonian and Carboniferous metamorphosed limestones and schists. Among other caves, there are the Zagedanskaya $(-570 \mathrm{~m}, 5,300 \mathrm{~m}$ long) and Alekseeva ( $-465 \mathrm{~m}, 4,410$ long).

In the Dzhentu Massif, the Mayskaya Cave is -500 deep and 4,410 $\mathrm{m}$ long.

In the alpine Fisht Massif at the western flank of the core zone and the westernmost summit of the Caucasus Major Range, explored from 1980 to 1996, deep caves in Upper Jurassic limestones are present: the Krestik-Turist ($633 \mathrm{~m}, 14 \mathrm{~km}$ long), Paryashchaya Ptitsa (-595 $\mathrm{m}, 4,500 \mathrm{~m}$ long) and Ol'ga (-520 m, 3,500 m long) caves; most springs are located at the range bottom 1,200 m a.s.l. (Reisner, Shelepin 1997; Klimchouk 2003a).

Numerous zones of gypsum karst are present along the northern macro-slope of the Great Caucasus. Many caves support subterranean streams. The longest are the Popova $(1,670 \mathrm{~m})$, Ammonal'naya $(1,460 \mathrm{~m})$ and Seteney $(980 \mathrm{~m})$ caves (Kazanbiev, 1975; Klimchouk etal., 1996; Makukhin, Molodkin, 1988; Ostapenko, 1993, 1994; Sukhovey, 1992).

On the northern macro-slope of the Caucasus Major, the most important biospeleological areas are located along the Black Sea in the Krasnodar Province (the Alek, Akhtsu, Voron- 
tsovky, Dzykhra, Akhshtyr, Akhun and FishtOshten-Lagonaki massifs of Jurassic dolomites). As of 2003, the longest and deepest caves are the Vorontsovkaya $(10.6 \mathrm{~km})$ and RucheinayaShkol'naya-Zabludshikh (-601 m) (Gvozdetski, 1965a, 1965b).

Cave fauna is relatively well known.

\section{Subterranean biota}

Birstein and Ljovuschkin (1950) divided Russia into two major biospeleological zones:

1. A northern zone covering most of the Russian territory: the Russian Plain and the northern Urals, both characterized by very poor subterranean faunas, with only a few stygobionts belonging to copepods, Bathynella syncarids and amphipods that correspond to old widespread groups.

2. A southern zone (Ciscaucasia and western Transcaucasia), characterized by numerous endemic stygo- and troglobionts.

About 100 exclusively subterranean taxa have been recorded from European Russia, more than $50 \%$ being aquatic. Most of them inhabit the Russian Great Caucasus.

Based on actual knowledge, there are three biospeological zones: central Russia, the Urals and the Russian Great Caucasus.

IV. 1. Valdai-Kuloisk. The northern part of the Russian Plain

Mostly microorganisms are surveyed in gypsum caves of the Belomorsko-Kuloiskoe Plateau: the Golubinskaya, Pekhorovskiy Proval and G-1 caves, all by Semikolennykh (1997). These gypsum caves are embedded 3-15 m deep under the surface and are very wet, with high-level flood waters annually. The mean temperature is about $2-8{ }^{\circ} \mathrm{C}$ in summer and nearly $0{ }^{\circ} \mathrm{C}$ in winter.

The following species have been identified, none of which represents troglo-or stygobionts: Domain Bacteria Woese, Kandler et Wheelis, 1990

Division Actinobacteria Goodfellow, 2012 Order Micromonosporales Genilloud, 2015
Family Micromonosporaceae Krassilnikow, 1938 em. Zhi, Li et Stackebrandt, 2009

- Micromonospora sp., from mineral clay samples;

Order Actinomycetales Buchanan, 1917

Family Streptomycetaceae Waksman et Henrici, 1943

- Streptomyces sp., from mineral clay samples;

Unidentified sulfate-reducing bacteria were also discovered.

Domain Eukaryota (Chatton, 1925) Whittaker et Margulis, 1978

Kingdom Fungi Linnaeus, 1753 stat. nov. Necker, 1783 em. Cavalier-Smith, 1998

Division Ascomycota Berkeley, 1857 stat. nov. Cavalier-Smith, 1987

Class Eurotiomycetes O.E. Eriksson et Winka, 1997

Order Eurotiales G.W.Martin ex Benny et Kimbrough, (1980)

Family Trichocomaceae E. Fischer, (1897)

- Penicillium italicum (Wehmer, 1894), on dead insects;

- Penicillium viridicatum Westling, 1911, with stable colonies on cave clay;

Class Dothideomycetes O.E. Eriksson et Winka, 1997

Order Pleosporales Luttrell ex M.E. Barr, (1987)

Family: Incertae sedis

- Pyrenochaeta sp.;

Division Zygomycota Barr, 1982

Order Mucorales Fries, 1832

Family Mucoraceae Dumortier, (1822)

- Rhizopus sp., on organic matter (flooded or penetrated through infiltration);

Kingdom Viridiplantae Cavalier-Smith, 1981

Division Chlorophyta Reichenbach, 1828, em.

Pascher, 1914, em. Lewis et McCourt, 2004

Order Chlorococcales Pascher, 1915

Family Chlorococcaceae Blackman et Tansley, 1902

- Chlorococcus sp., forming green spots near the entrance, always situated in reflected light.

Kingdom Protozoa R. Owen, 1858

Subkingdom Sarcomastigota Cavalier-Smith, 1983 
Group Excavata (Cavalier-Smith), 2002

Phylum Amoebozoa Lühe, 1913

Class Tubulinea Smirnov, Nassonova, Berney,

Fahrni, Bolivar et Pawlowski, 2005

Order Arcellinida Kent, 1880

Family Centropyxidae Jung, 1942

- Centropyxis aerophila Deflandre, 1929.

Found in a cave at the bank of Pechora River within the Pechoro-Ilychskiy Nature Reserve (Troitsk-Pechorsky District, Komi Republic) (Mazei et al., 2012).

Family Phryganellidae Jung, 1942

- Phryganella hemisphaerica Penard, 1902.

Found in a cave at the bank of Pechora River within the Pechoro-Ilychskiy Nature Reserve (Troitsk-Pechorsky District, Komi Republic) (Mazei et al., 2012).

Kingdom Animalia Linnaeus, 1758

Phylum Nematoda Diesing, 1861

Nematoda indet. Unidentified nematodes have been recorded in the caves Olimpiyskaya and Muzeynaya, Pinezhskiy Nature Reserve, Arkhangelsk Region (Chertoprud et al., 2011).

Phylum Annelida Lamarck, 1809

Class Oligochaeta Grube, 1850

Order Enchytraeida Vejdovský, 1879

Family Enchytraeidae Vejdovský, 1879

Enchytraeidae indet. Unidentified enchytraeids have been recorded in the Olimpiyskaya Cave, Pinezhskiy Nature Reserve, Arkhangelsk Region (Chertoprud et al., 2011).

Phylum Arthropoda Siebold, 1848

Subphylum Crustacea Brünnich, 1772

Class Copepoda Milne-Edwards, 1840

Order Cyclopoida Burmeister, 1834

Family Cyclopidae Rafinesque, 1815

- Megacyclops viridis (Jurine, 1820). Recorded in waterbodies inside the Muzeynaya Cave, Pinezhskiy Nature Reserve, Arkhangelsk Region (Chertoprud et al., 2011).

Class Malacostraca Latreille, 1817

Order Amphipoda Latreille, 1816

Family Pallaseidae Tachteew, 2000

- Pallasea sp. Recorded in waterbodies inside the Kitezh Cave, Pinezhskiy Nature
Reserve, Arkhangelsk Region (Sidorov et al., 2011; Chertoprud et al., 2011).

Class Arachnida Cuvier, 1812

Subclass Acari Leach, 1817

The fauna of free-living gamasid mites of the Pinezhskiy Nature Reserve is unusually diverse and rich (117 species) (Makarova, 2009), but its caves support only few presumed trogloxenes. Apparently, the caves are too cold to sustain a characteristic terrestrial fauna.

Superclass Hexapoda Blainville, 1816

Class Collembola Lubbock, 1870

The springtail fauna of the Pinezhskiy Nature Reserve is unusually diverse and rich (126 species) (Babenko, 2008), but its caves support only few presumed trogloxenes. Apparently, the caves are too cold to sustain a characteristic terrestrial fauna.

Class Insecta Linnaeus, 1758

Order Ephemeroptera Hyatt et Arms, 1891

Family Baetidae Leach, 1815

- Baetis rhodani (Pictet, 1843). Recorded in waterbodies inside the Olimpiyskaya Cave, Pinezhskiy Nature Reserve, Arkhangelsk Region (Chertoprud et al., 2011).

- Centroptilum luteolum Müller, 1776. Recorded in waterbodies inside the Olimpiyskaya and Muzeynaya caves, Pinezhskiy Nature Reserve, Arkhangelsk Region (Chertoprud et al., 2011).

Family Siphlonuridae Ulmer, 1920

- Siphlonurus lacustris Eaton, 1870. Recorded in waterbodies inside the Muzeynaya Cave, Pinezhskiy Nature Reserve, Arkhangelsk Region (Chertoprud et al., 2011).

Order Plecoptera Burmeister, 1839

Family Nemouridae Newman, 1853 - Nemurella pictetii (Klapálek, 1900). Recorded in waterbodies inside the Olimpiyskaya Cave, Pinezhskiy Nature Reserve, Arkhangelsk Region (Chertoprud et al., 2011).

- Nemoura sahlbergi Morton, 1896. Recorded in waterbodies inside the Olimpiyskaya Cave, Pinezhskiy Nature Reserve, Arkhangelsk Region (Chertoprud et al., 2011). 
- Nemoura aff. avicularis Morton, 1894. Recorded in waterbodies inside the Muzeynaya Cave, Pinezhskiy Nature Reserve, Arkhangelsk Region(Chertoprudetal., 2011). Family Perlodidae Klapálek, 1912

- Diura nanseni (Kempny, 1900). Recorded in waterbodies inside the Olimpiyskaya Cave, Pinezhskiy Nature Reserve, Arkhangelsk Region (Chertoprud et al., 2011).

Family Taeniopterygidae Klapálek, 1905

- Taeniopteryx nebulosa (Linnaeus, 1758). Recorded in waterbodies inside the Olimpiyskaya and Muzeynaya caves, Pinezhskiy Nature Reserve, Arkhangelsk Region (Chertoprud et al., 2011).
Order Hemiptera Linnaeus, 1758

Family Corixidae Leach, 1815

- Sigara semistriata (Fieber, 1848). Recorded in waterbodies inside the Olimpiyskaya Cave, Pinezhskiy Nature Reserve, Arkhangelsk Region (Chertoprud et al., 2011).

Order Diptera Linnaeus, 1758

Suborder Nematocera Schiner, 1862

Family Limoniidae Speiser, 1909

- Eloeophila mundata (Loew, 1871). Recorded in waterbodies inside the Olimpiyskaya Cave, Pinezhskiy Nature Reserve, Arkhangelsk Region (Chertoprud et al., 2011).

Table 1. List of *stygobionts, stygophile and troglophile species from the northern part of the Russian Plain.

Таблица 1. Список * стигобионтов, стигофилов и троглофилов из северной части Русской равнины.

A - Stygobionts*: stygophiles

BACTERIA

Micromonosporaceae

Micromonospora sp.

Streptomycetaceae

Streptomyces sp.

FUNGI

Trichocomaceae

Penicillium italicum (Wehmer, 1894);

Penicillium viridicatum Westling, 1911

Pleosporales

Pyrenochaeta sp.

Mucoraceae

Rhizopus sp.

VIRIDIPLANTAE

Chlorococcaceae

Chlorococcus sp.

PROTOZOA

Centropyxidae

Centropyxis aerophila Deflandre, 1929

Phryganellidae

Phryganella hemisphaerica Penard, 1902

NEMATODA

Nematoda indet.

OLIGOCHAETA

Enchytraeidae indet.

COPEPODA

Cyclopidae

Megacyclops viridis (Jurine, 1820)

Pallaseidae

Pallasea sp.

INSECTA

\section{EPHEMEROPTERA}

Siphlonuridae

Siphlonurus lacustris Eaton, 1870

Baetidae

Centroptilum luteolum Müller, 1776;

Baetis rhodani (Pictet, 1843)

PLECOPTERA

Perlodidae

Diura nanseni (Kempny, 1900)

Taeniopterygidae

Taeniopteryx nebulosa (Linnaeus, 1758)

Nemouridae

Nemurella pictetii (Klapálek, 1900);

Nemoura sahlbergi Morton, 1896;

Nemoura af. avicularis Morton, 1894

HETEROPTERA

Corixidae

Sigara semistriata (Fieber, 1848)

DIPTERA

Pediciidae

Dicranota bimaculata (Schummel, 1829)

Limoniidae

Eloeophila mundata (Loew, 1871)

Simuliidae

Cnetha sp.

Chironomidae

Guttipelopia guttipennis (Wulp, 1874)

CHIROPTERA

Vespertilionidae

Myotis brandtii (Eversmann, 1845)

Eptesicus nilssonii (Keyserling et Blasius, 1839) 
Family Pediciidae Osten-Sacken, 1860

- Dicranota bimaculata (Schummel, 1829).

Recorded in waterbodies inside the Olimpiyskaya Cave, Pinezhskiy Nature Reserve, Arkhangelsk Region (Chertoprud et al., 2011).

Family Simuliidae Newman, 1834

-Cnetha sp. Recorded in waterbodies inside the Olimpiyskaya Cave, Pinezhskiy Nature Reserve, Arkhangelsk Region(Chertoprud et al., 2011).

Family Chironomidae Jacobs, 1900

- Guttipelopia guttipennis (Wulp, 1874). Recorded in waterbodies inside the Muzeynaya Cave, Pinezhskiy Nature Reserve, Arkhangelsk Region (Chertoprud et al., 2011).

Phylum Chordata Haeckel, 1874

Subphylum Vertebrata J.-B. Lamarck, 1801

Class Mammalia Linnaeus, 1758

Order Chiroptera Blumenbach, 1779

Famaly Vespertilionidae Gray, 1821

- Myotis brandtii (Eversmann, 1845). Solitarily hibernating individuals of this species were reported from the Golubinskiy Proval Cave, Pinezhskiy Nature Reserve, where they were found twice in 1980 (Rykov, 2014).

- Eptesicus nilssonii (Keyserling et Blasius, 1839). Hibernating Northern bats were reported from several karst caves of the Belomorsko-Kuloiskoe Plateau, with the maximum number of 16 bats observed in one visit to the Golubinskiy Proval Cave (Rykov, 2014).

\section{2. Central Russia}

The cave biota of central Russia is littleknown and poorly studied. Below is only a preliminary evaluation.

This karstic region lies too northerly to be subjected to either Mediterranean or Balkan, or Asiatic influence.

Bacteria, Algae and Fungi were surveyed in the Poneretka Cave, Borovichskiy District, Novgorod Region, located in limestones of the Valdai Plateau, temperature $9{ }^{\circ} \mathrm{C}$, by Semikolennykh (1997). The numbers of microorgan- isms are about $10^{7}$ per sediment gram.

Domain Bacteria Woese, Kandler et Wheelis, 1990

Division Actinobacteria Goodfellow, 2012

Order Actinomycetales Buchanan, 1917

Family Mycobacteriaceae Chester, 1897

- Mycobacterium hyalinum Söhngen, 1913;

- M. licheniforme Krassilnikow, 1949;

- M. luteum Söhngen, 1913;

- M. mucosum Krassilnikow, 1941

Family Propionibacteriaceae Delwiche, 1957

- Propionibacterium sp.

Family Streptomycetaceae Waksman et Henrici, 1943

- Streptomyces sp.;

- Streptoverticillium sp.;

- Streptosporangium sp.

Family Micrococcaceae Pribram, 1929

- Arthrobacter globiformis Conn et Dimmick, 1947

Class Bacilli Ludwig, Schleifer et Whitman, 2010

Order Bacillales Prévot, 1953

Family Bacillaceae Fischer, 1895

- Bacillus mycoides Flügge, 1886

Division Cyanobacteria Stanier, 1973

Order Nostocales Cavalier-Smith, 2002

Family Nostocaceae C.A. Agardh, 1824 ex

Kirchner, 1898

- Nostoc sp.

Division Proteobacteria Stackebrandt, Murray et Trüper, 1988, Garrity, Bell et Lilburn, 2005

Class Gammaproteobacteria Garrity, Bell et Lilburn, 2005

Order Pseudomonadales Orla-Jensen, 1921

Family Pseudomonadaceae Winslow, Broadhurst, Buchanan, Krumwiede, Rogers et Smith, 1917

- Pseudomonas desmolyticum Cray et Thornton, 1928

Kingdom Fungi Linnaeus, 1753 stat. nov. Necker, 1783 em. Cavalier-Smith, 1998

Division Ascomycota Berkeley, 1857 stat. nov. Cavalier-Smith, 1987

Class Eurotiomycetes O.E. Eriksson et Winka, 1997

Order Onygenales Ciferri ex Benny et Kimbrough, (1980) 
Family Gymnoascaceae Baranetsky, (1872) - Pseudoarachniotus sp.

Kingdom Viridiplantae Cavalier-Smith, 1981 Division Chlorophyta Reichenbach, 1828, em. Pascher, 1914, em. Lewis et McCourt, 2004

Class Trebouxiophyceae Friedl, 1995

Chlorellales sp. is the only species collected.

Protists are extremely poorly known in caves of the central part of European Russia. Only two papers deal with this group in the region in question. One is by Semikolennykh (1997), dedicated to the study of Protista in the Poneretka Cave, Borovichskiy District, Novgorod Region. The other is by Mazei et al. (2012), which focuses on microfauna in artificial adits of the Zhiguli Hills, Stavropolskiy District, Samara Region.

Kingdom Chromista Cavalier-Smith, 1981

Phylum Ciliophora Doflein, 1901

Class Oligohymenophorea de Puytorac et al., 1974

Order Pleuronematida Fauré-Fremiet in Corliss, 1956

Family Pleuronematidae Kent, 1881

- Balantiophorus elongatus Schewiakoff, 1892. Recorded from the Poneretka Cave, Borovichskiy District, Novgorod Region (Semikolennykh, 1997).

Kingdom Protozoa R. Owen, 1858

Subkingdom Sarcomastigota Cavalier-Smith, 1983

Group Excavata (Cavalier-Smith), 2002

Phylum Euglenozoa Cavalier-Smith, 1981

Class Euglenophyceae Schoenichen, 1925

Order Peranemida Bütschli, 1884

Family Bodonaceae Bütschli, 1884

- Bodo globosus Stein, 1878. Recorded from the Poneretka Cave, Borovichskiy District, Novgorod Region (Semikolennykh, 1997).

- B. lens (O.F. Müller) Klebs (1892). Recorded from the Poneretka Cave, Borovichskiy District, Novgorod Region (Semikolennykh, 1997).

- Bodo sp. Recorded from the Poneretka Cave, Borovichskiy District, Novgorod Region (Semikolennykh, 1997).
Phylum Amoebozoa Lühe, 1913

Class Tubulinea Smirnov, Nassonova, Berney,

Fahrni, Bolivar et Pawlowski, 2005

Order Arcellinida Kent, 1880

Family Arcellidae Ehrenberg, 1843

- Arcella arenaria compressa Chardez,

1974. Recorded from artificial adits of the Zhiguli Hills, Stavropolskiy District, Samara Region (Mazei et al., 2012).

Family Centropyxidae Jung, 1942

- Centropyxis aerophila Deflandre, 1929. Recorded from artificial adits of the Zhiguli Hills, Stavropolskiy District, Samara Region (Mazei et al., 2012).

- C. aerophila sphagnicola Deflandre, 1929. Recorded from artificial adits of the Zhiguli Hills, Stavropolskiy District, Samara Region (Mazei et al., 2012).

- C. plagiostoma Thomas et Bonnet, 1955. Recorded from the Poneretka Cave, Borovichskiy District, Novgorod Region (Semikolennykh, 1997).

Family Hyalospheniidae Schultze, 1877

- Hyalosphenia papilio Leidy, 1879. Recorded from artificial adits of the Zhiguli Hills, Stavropolskiy District, Samara Region (Mazei et al., 2012).

Family Nebelidae Taranek, 1882

- Nebela parvula Cash, 1909. Recorded from artificial adits of the Zhiguli Hills, Stavropolskiy District, Samara Region (Mazei et al., 2012).

- N. tincta Leidy, 1879. Recorded from artificial adits of the Zhiguli Hills, Stavropolskiy District, Samara Region (Mazei et al., 2012).

Family Phryganellidae Jung, 1942

- Phryganella acropodia (Hertwig et Lesser, 1874). Recorded from artificial adits of the Zhiguli Hills, Stavropolskiy District, Samara Region (Mazei et al., 2012).

- P. hemisphaerica Penard, 1902. Recorded from artificial adits of the Zhiguli Hills, Stavropolskiy District, Samara Region (Mazei et al., 2012).

Phylum Amoebozoa Lühe, 1913 em. CavalierSmith, 1998

Class Archamoebea Cavalier-Smith, 1998

Family Mastigamoebidae Goldschmidt, 1907 - Mastigamoeba sp. Recorded from the Poneretka Cave, Borovichskiy District, Novgorod Region (Semikolennykh, 1997). 
Class Tubulinea Smirnov, Nassonova, Berney, Fahrni, Bolivar et Pawlowski, 2005

Order Tubulinida Smirnov, Nassonova, Berney, Fahrni, Bolivar et Pawlowski, 2005

Family Hartmannellidae Volkonsky, 1931

- Hartmannella sp. Recorded from the Poneretka Cave, Borovichskiy District, Novgorod Region (Semikolennykh, 1997).

Kingdom Rhizaria Cavalier-Smith, 2002

Phylum Cercozoa Cavalier-Smith, 1998

Class Imbricatea Cavalier-Smith, 2003

Order Euglyphida Copeland, 1956

Family Euglyphidae Wallich, 1864

- Assulina seminulum (Ehrenberg, 1848). Recorded from artificial adits of the Zhiguli Hills, Stavropolskiy District, Samara Region (Mazei et al., 2012).

- Euglypha rotunda Wailes, 1915. Recorded from artificial adits of the Zhiguli Hills, Stavropolskiy District, Samara Region (Mazei et al., 2012).

Family Trinematidae Hoogenraad et de Groot, 1940

- Trinema complanatum Penard, 1890. Recorded from artificial adits of the Zhiguli Hills, Stavropolskiy District, Samara Region (Mazei et al., 2012).

- T. enchelys (Ehrenberg, 1838). Recorded from artificial adits of the Zhiguli Hills, Stavropolskiy District, Samara Region (Mazei et al., 2012).

Kingdom Animalia Linnaeus, 1758

Phylum Rotifera Cuvier, 1817

Phylum Annelida Lamarck, 1809

Class Oligochaeta Grube, 1850

Order Crassiclitellata Jamieson, 1988

Family Lumbricidae Claus, 1876

- Eisenia foetida (Savigny, 1826), first recorded by Michaelsen in 1926 (Malevich, 1947) from moist clay in the deep part of a cave on the right bank of Volga River, downstream the mouth of Kama River. It was found again by Birstein in several caves of the Russian Caucasus and in Abkhazia. The species is trogloxene in Western Europe and abundant in farmyard manure. It is perhaps troglophilic in Russian and Georgian caves with bat guano.
- Eiseniella tetraedra (Savigny, 1826). Recorded from wells in the city of Saratov (Behning, 1928).

Order Enchytraeida Vejdovský, 1879

Family Enchytraeidae Vejdovský, 1879

- Henlea ventriculosa (d'Udekem, 1854).

Recorded from wells in the city of Saratov (Behning, 1928).

- Enchytraeus albidus Henle, 1837. Recorded from wells in the city of Saratov (Behning, 1928).

- Fridericia bulbosa (Rosa, 1887). Recorded from wells in the city of Saratov (Behning, 1928).

Order Tubificida Brinkhurst, 1982

Family Naididae Ehrenberg, 1828

- Nais communis Piguet, 1906. Repeatedly recorded from numerous wells in European Russia, including those in the city of Saratov (Behning, 1928).

- N. pseudobtusa Piguet, 1906. Recorded from wells in the city of Saratov (Behning, 1928).

Family Tubificidae Vejdovský, 1884

- Potamothrix hammoniensis (Michaelsen,

1901). Recorded from wells in the city of

Saratov (Behning, 1928).

Phylum Nematoda Diesing, 1861

Nematodes have almost never been studied in caves of the central part of European Russia. The only relevant paper is that by Behning (1928) which puts on record 11 species of the group found in wells in the city of Saratov. This city is located on the Volga Upland within the Saratov Region:

Class Enoplea Inglis, 1983

Order Monhysterida Filipjev, 1929

Family Dorylaimidae De Man, 1876

- Eudorylaimus carteri (Bastian, 1865);

- E. carteri rotundatus (Micoletzky, 1922);

- E. monohystera (De Man, 1880);

- E. obtusicaudatus (Bastian, 1865);

- Laimydorus pseudostagnalis (Micoletzky, 1927).

Family Monhysteridae De Man, 1876

- Monhystera paludicola De Man, 1881.

Order Mononchida Jairajpuri, 1969

Family Mononchidae Chitwood et Chitwood, 1937

- Mononchus macrostoma Bastian, 1865;

- M. papillatus Bastian, 1865. 
Order Triplonchida Cobb, 1920

Family Tobrilidae Filipjev, 1918

- Tobrilus allophysoides (Micoletzky, 1925).

Class Chromadorea Inglis, 1932

Family Rhabditidae Örley, 1880

- Rhabditis curvicaudata (Schneider, 1866).

Class Secernentea Lorenzen, 1981

Order Tylenchida Thorne, 1949

Family Cephalobidae Filipjev, 1934

- Cephalobus rigidus Schneider, 1866.

Phylum Arthropoda Siebold, 1848

Subphylum Crustacea Brünnich, 1772

Class Copepoda Milne-Edwards, 1840

Order Cyclopoida Burmeister, 1834

- Diacyclops bicuspidatus (Claus, 1857).

Stygoxene, recorded from the Bornukovskaya Cave in the Nizhniy Novgorod Region (Birstein, Ljovuschkin, 1966).

- Acanthocyclops vernalis (Fischer, 1853).

Stygophile, recorded from the Bornukovskaya Cave in the Nizhniy Novgorod Region (Birstein, Ljovuschkin, 1966).

Order Harpacticoida G.O. Sars, 1903

Family Canthocamptidae Brady, 1880

- Canthocamptus staphylinus (Jurine, 1820). Stygoxene, recorded from wells near the city of Saratov (Behning, 1928).

Family Parastenocarididae Chappuis, 1940

- Parastenocaris fonticola Borutzky, 1926, decribed from a well at Kosino near Moscow City (Borutzky, 1926), currently known to be a widespread species (Borutzky, 1926).

Class Ostracoda Latreille, 1802

Order Podocopida G.O. Sars, 1866

Family Cyprididae Baird, 1845

- Potamocypris wolfi Brech, 1920. Crenobiont, found in underground streams near the city of Voronezh below Tertiary sandstone plates (Bronstein, 1947).

Class Malacostraca Latreille, 1817

Superorder Syncarida Packard, 1885

Order Bathynellacea Chappuis, 1915

Family Bathynellidae Grobbon, 1904

- Bathynella sp. Formerly referred to as Bathynella chappuisi Delachaux, 1919, from a well $65 \mathrm{~km}$ W of Kalach-on-Don, Volgograd (Schäfer, 1951). The species identity requires confirmation (Birstein, Ljovuschkin, 1967b).

Order Amphipoda Latreille, 1816

Family Crangonyctidae Bousfield, 1973

- Synurella ambulans (F. Müller, 1847)

(= S. meschtscherica Borutzky, 1929). Stygophile, widespread across European Russia. Recorded from springs in the Ryazan (Borutzky, 1927), Moscow (Chertoprud, 2006), Vladimir, Kaluga, Pskov and Bryansk regions (Sidorov, Palatov, 2012).

- Synurella derzhavini Behning, 1928. Stygobiont, eyes and integument unpigmented. Described from wells near the city of Saratov (Behning, 1928).

- Synurella donensis (Martynov, 1919). Stygobiont, described from springs in the Kiziterinka River valley, now within the city of Rostov-on-Don (Martynov, 1919).

Class Arachnida Cuvier, 1812

Order Aranei Clerck, 1758

Family Nesticidae Simon, 1894

- Nesticus cellulanus (Clerck, 1758). Troglophile, found in caves at Kamennoe, Nizhniy Novgorod Region (Kapralov, Chernorudskiy, 2009).

Family Tetragnathidae Menge, 1866

- Metellina merianae (Scopoli, 1763). Troglophile, widespread in Europe at cave entrances, found in caves at Kamennoe, Nizhniy Novgorod Region (Kapralov, Chernorudskiy, 2009).

Superclass Hexapoda Blainville, 1816

Class Insecta Linnaeus, 1758

Order Coleoptera Linnaeus, 1758

Family Cryptophagidae Kirby, 1937

- Cryptophagus setulosus Sturm, 1845. Troglophile, recorded from caves in the Novgorod Region (Kapralov, 2015).

- C. schmidti Sturm, 1845. Troglophile, recorded from caves of the Novgorod Region (Kapralov, 2015).

- Henoticus serratus (Gyllenhal, 1808). Troglophile, recorded from the Baskunchakskaya Cave, Akhtubinskiy District, Astrakhan Region (Kapralov, 2015).

Family Leiodidae Fleming, 1821

- Catops morio (Fabricius, 1792). Troglophile, recorded from caves in the Novgorod Region (Kapralov, 2015). 
- Choleva glauca (Britten, 1918). Subtroglophile, recorded from caves in the Novgorod Region (Kapralov, 2015).

- Ch. lederiana Reitter, 1902. Subtroglophile, a mass species both in natural and artificial caverns in the Novgorod Region, also reported from quarries in the Tver and Ryazan regions (Kapralov, 2015).

- Cholevinus pallidus (Ménétriés, 1832). Subtroglophile, recorded in a cave near Lake Baskunchak, Akhtubinskiy District, Astrakhan Region (Perkovsky, 1991).

Order Lepidoptera Linnaeus, 1758

Family Noctuidae Latreille, 1809

- Scoliopteryx libatrix (Linnaeus, 1758). Troglophile, recorded in European Russia from numerous caves in the Novgorod and Astrakhan regions (Kapralov, Chernorudskiy, 2009; Mukhanov, Kapralov, 2010; Kapralov, 2015).

Order Diptera Linnaeus, 1758

Suborder Nematocera Schiner, 1862

Family Trichoceridae Rondani, 1841

- Trichocera maculipennis Meigen, 1818.

Troglophile, common in caves of European

Russia in the Novgorod and Astrakhan regions, as well as the Perm Province (Kapralov, Chernorudskiy, 2009; Pan'kov et al., 2009b; Kapralov, 2015).

- Trichocera regelationis (Linnaeus, 1758). Troglophile, common in caves of European Russia in the Novgorod and Astrakhan regions (Kapralov, Chernorudskiy, 2009; Kapralov, 2015).

Suborder Brachycera Zetterstedt, 1842

Family Phoridae Gray, 1840

Phoridae gen. spp. Likely troglophiles, larvae found in numerous caves of European

Russia, but identified closer to neither genera nor species (Kapralov, 2015).

Phylum Chordata Haeckel, 1874

Subphylum Vertebrata J.-B. Lamarck, 1801

Class Mammalia Linnaeus, 1758

Order Chiroptera Blumenbach, 1779

Family Vespertilionidae Gray, 1821

Strictly speaking, only a few records of bats have been made in natural undergrounds due to scarcely available karst caves. Hence, the ma- jority of findings refer to mines, cellars, rockcut cells and cave monasteries. In many cases naturally presented cavities and crevices were deepened and transformed into artificial underground chambers; therefore, it is virtually impossible to estimate the past importance of these roosts to bats. Whereas no bats reproduce in central Russia, underground chambers, regardless of their origin, represent the only kind of winter shelter suitable for the hibernation of seven resident species. Aside from many dozens of other records from various underground roosts, all seven of these species were recorded from the natural caves of the Ichalkovskiy Nature Reserve, Novgorod Region, where they have been either netted at entrances during swarming or observed hibernating (Bakka, Bakka, 1999):

- Myotis dasycneme (Boie, 1825);

- Myotis daubentonii (Kuhl, 1817);

- Myotis nattereri (Kuhl, 1817);

- Myotis brandtii (Eversmann, 1845);

- Myotis mystacinus (Kuhl, 1817);

- Plecotus auritus (Linnaeus, 1758);

- Eptesicus nilssonii (Keyserling et Blasius, 1839).

Conclusion. The terrestrial and aquatic cave biota in central Russia is scarce because the Quaternary glaciations must have destroyed all old cave species. Apparently, the only way for survival was in subterranenan phreatic and hyporheic habitats (Table 2) (Kniss, Smirnov, 1990; Kniss, 2001, 2004).

\section{3. Ural}

In the Urals, caves are relatively well studied compared to northern Russia's caverns. Microarthropod cave fauna is diversified. The subterranean fauna consists of numerous troglophilic species, as well as several troglobionts and stygobionts. Troglobionts are known only amongst Collembola.

Studies on microorganisms in the sediment of the Ledyanaya and Kungurskaya ice caves in the central Urals show them to range from 44,000 to 500,000 ex. per gram from winter to spring (Volodin, Pshenichnikov, 1949). 
Table 2. List of *stygobionts, stygophile and troglophile species from central Russia. Таблица 2. Список *стигобионтов, стигофильных и троглофильных видов A - Stygobionts *: stygophiles из Центральной России.

\section{BACTERIA}

Mycobacteriaceae

Mycobacterium hyalinum Söhngen, 1913

Mycobacterium licheniforme Krassilnikow, 1949

Mycobacterium luteum Söhngen, 1913

Mycobacterium mucosum Krassilnikow, 1941

Propionibacteriaceae

Propionibacterium sp.

Streptomycetaceae

Streptomyces sp.

Streptoverticillium sp.

Streptosporangiaceae

Streptosporangium sp.

Micrococcaceae

Arthrobacter globiformis Conn et Dimmick, 1947

Bacillaceae

Bacillus mycoides Flügge, 1886

Nostocaceae

Nostoc sp.

Pseudomonadaceae

Pseudomonas desmolyticum Cray et Thornton, 1928

FUNGI

Gymnoascaceae

Pseudoarachniotus sp.

VIRIDIPLANTAE

Chlorophyta: Trebouxiophyceae

Chlorellales sp.

CILIOPHORA

Pleuronematidae

Balantiophorus elongatus Schewiakoff, 1892

EUGLENOZOA

Bodonaceae

Bodo globosus Stein, 1878

Bodo lens (O.F. Müller) Klebs, (1892)

Bodo sp.

RHIZARIA

Euglyphidae

Assulina seminulum (Ehrenberg, 1848)

Euglypha rotunda Wailes, 1915

Trinematidae

Trinema complanatum Penard, 1890

Trinema enchelys (Ehrenberg, 1838)

AMOEBOZOA

B - Troglobionts*: troglophiles

Oligochaeta

Lumbricidae

Eisenia foetida Savigny, 1826

Eiseniella tetraedra (Savigny, 1826)

Aranei

Tetragnatidae

Metellina merianae (Scopoli, 1763)
Arcellidae

Arcella arenaria compressa Chardez, 1974

Centropyxidae

Centropyxis aerophila Deflandre, 1929

Centropyxis aerophila sphagnicola Deflandre, 1929

Centropyxis plagiostoma Thomas et Bonnet, 1955

Hyalospheniidae

Hyalosphenia papilio Leidy, 1879

Nebelidae

Nebela parvula Cash, 1909

Nebela tincta Leidy, 1879

Phryganellidae

Phryganella acropodia (Hertwig et Lesser, 1874)

Phryganella hemisphaerica Penard, 1902

Mastigamoebidae

Mastigamoeba sp.

Hartmannellidae

Hartmannella sp.

Oligochaeta

Enchytraeidae

Henlea ventriculosa (d'Udekem, 1854)

Enchytraeus albidus Henle, 1837

Fridericia bulbosa (Rosa, 1887)

Haplotaxida: Naididae

Nais communis Piguet, 1906

Nais pseudobtusa Piguet, 1906

Haplotaxida: Tubificidae

Potamothrix hammoniensis (Michaelsen, 1901)

COPEPODA

Cyclopoida: Cyclopidae

Acanthocyclops vernalis Fischer, 1853

Harpacticoida: Parastenocarididae

*Parastenocaris fonticola Borutzky, 1926

OSTRACODA

Podocopida: Cyprididae

Potamocypris wolfi Brech, 1920

Syncarida

Bathynellacea: Bathynellidae

*Bathynella sp.

Amphipoda

Crangonyctidae

*Synurella derzhavini Behning, 1925

*Synurella donensis (Martynov, 1919)

Synurella ambulans (F. Müller, 1846)

Nesticidae

Nesticus cellulanus (Clerck, 1758)

COLEOPTERA

Cryptophagidae

Cryptophagus setulosus Sturm, 1845

Cryptophagus schmidti Sturm, 1845

Henoticus serratus (Gyllenhal, 1808) 
Leiodidae

Catops morio (Fabricius, 1792)

Choleva glauca (Britten, 1918)

Choleva lederiana Reitter, 1902

Cholevinus pallidus (Ménétriés, 1832)

LEPIDOPTERA

Noctuidae

Scoliopteryx libatrix (Linnaeus, 1758)

DIPTERA

Trichoceridae

Trichocera maculipennis Meigen, 1818

Trichocera regelationis (Linnaeus, 1758)
Phoridae

Phoridae gen. spp.

CHIROPTERA

Vespertilionidae

Myotis dasycneme (Boie, 1825)

Myotis daubentonii (Kuhl, 1817)

Myotis nattereri (Kuhl, 1817)

Myotis brandtii (Eversmann, 1845)

Myotis mystacinus (Kuhl, 1817)

Plecotus auritus (Linnaeus, 1758)

Eptesicus nilssonii (Keyserling et Blasius, 1839)
Phylum Annelida Lamarck, 1809

Class Oligochaeta Grube, 1850

Order Crassiclitellata Jamieson, 1988

Family Lumbricidae Claus, 1876

- Dendrobaena octaedra (Savigny, 1826).

Troglophile, recorded from the Shulgan-

Tash Cave, Burzianskiy District, Bashkortostan (= Republic of Bashkiria) (Kniss, 1984b, 2001).

- Dendrodrilus rubidus (Savigny, 1826). Troglophile, recorded from caves of Bashkortostan (Kniss, 2001).

- Eiseniella tetraedra (Savigny, 1826). Likely troglophile, recorded from the Shulgan-Tash Cave, Burzianskiy District, Bashkortostan (Kniss, 1984b, 2001).

Phylum Arthropoda Siebold, 1848

Subphylum Crustacea Brünnich, 1772

Class Malacostraca Latreille, 1817

Order Amphipoda Latreille, 1816

Family Crangonyctidae Bousfield, 1973

- Crangonyx chlebnikovi chlebnikovi Borutzky, 1928 (= Crangonyx chlebnikovi maximovitshi Pankov et Pankova, 2004). Stygobiont, found in several caves in the Perm Province of the Urals (Pan'kov, 2008b; Pan'kov, Pan'kova, 2004; Pan'kov, Starova, 2009a, 2009c; Pan'kov et al., 2005, 2010, 2011; Sidorov et al., 2010, 2012).

Class Arachnida Cuvier, 1812

Order Aranei Clerck, 1758

Family Linyphiidae Blackwall, 1859

-Improphantes improbulus (Simon, 1929). Troglophile, recorded from the Kurmanaevskaya and Grot Tashastinskiy caves, both in Bashkortostan (Esyunin, Efimik, 1999).
- Megalepthyphantes pseudocollinus Saaristo, 1997. Troglophile, recorded from the Kungurskaya Cave in the Perm Province (Pan'kov, 2008a; Pan'kov et al., 2009b).

- Neriene montana (Clerck, 1758). Troglophile, recorded from the Kungurskaya Cave in the Perm Province (Pan'kov, 2008a; Pan'kov et al., 2009b).

- Neriene radiata (Walckenaer, 1841). Troglophile, recorded from the Chudesnitsa Cave in the Perm Province (Pan'kov, 2008a; Pan'kov et al., 2009b).

Family Nesticidae Simon, 1894

- Nesticus cellulanus (Clerck, 1758). Troglophile, recorded from the Shulgan-Tash (= Kapovaya) Cave in Bashkortostan (Abdullin et al., 2012), as well as in the Obvalnaya and Letuchikh Myshey caves in the Perm Province (Pan'kov et al., 2009).

Subclass Acari Leach, 1817

Superorder Acariformes Zakhvatkin, 1952

Order Prostigmata Kramer, 1877

Suborder Anystina Van der Hammen, 1972

Family Rhagidiidae Oudemans, 1922

- Rhagidia breviseta Zacharda, 1995. Apparently troglobiont, recorded from the Shulgan-Tash (= Kapovaya) Cave in Bashkortostan (Kapralov, 2015).

- Rhagidia sp. Apparently troglobiont, recorded from several caves in Bashkortostan, southern Urals, Russia (Kniss, 1984b, 2001). - Foveacheles sp. Troglobiont, recorded from the Shulgan-Tash (= Kapovaya) Cave in Bashkortostan (Abdullin et al., 2012; Kapralov, 2015). 
Suborder Eleutherengona Oudemans, 1909

Family Pygmephoridae Cross, 1965

- Pygmephorus sp. Troglophile, recorded from several caves in Bashkortostan, southern Urals (Kniss, 1984b, 2001).

Order Sarcoptiformes Reuter, 1909

Suborder Oribatida Van der Hammen, 1968

Family Aleurodamaeidae Paschoal et Johnston, 1984

- Epidamaeus sp. Troglophile, recorded from several caves in Bashkortostan, southern Urals (Kniss, 1984b, 2001).

Family Mochlozetidae Grandjean, 1960

- Sphaerobates sp. Troglophile, recorded from several caves in Bashkortostan, southern Urals (Kniss, 1984b, 2001).

Superclass Hexapoda Blainville, 1816

Class Collembola Lubbock, 1870

As many as 34 troglophile and 4 troglobiont species of springtails are known from Ural caves (Table 3).

Order Poduromorpha Börner, 1913, sensu D'Haese, 2002

Superfamily Neanuroidea Massoud, 1967, sensu D'Haese, 2002

Family Neanuridae Börner, 1906

- Philotella olgae Kniss et Thibaud, 1995.

Likely troglobiont, described and only known from caves in Bashkortostan (Kniss, Thibaud, 1995; Kniss, 2001, 2006).

Superfamily Hypogastruroidea Salmon, 1964, sensu Deharveng, 2004

Family Hypogastruridae Börner, 1906

- Schaefferia baschkirica Kniss, 1985. Troglobiont, described from the Klyka and Kiekbayevskaya caves, both on the right bank of Belaya River, as well as from the Kanskaya-2 Cave on the left bank of Kana River, all in Bashkortostan (Kniss, 1985, 2001; Babenko et al., 1994).

- Ceratophysella kapoviensis (Babenko, 1994). Troglobiont with faint traces of troglomorphism. Described from the ShulganTash (= Kapovaya) Cave in Bashkortostan (Babenko et al., 1994; Kniss, 2001).

Order Entomobryomorpha Börner, 1916, sensu Soto-Adames et al., 2008

Superfamily Isotomoidea Szeptycki, 1979, sensu Soto-Adames et al., 2008
Family Isotomidae Schäffer, 1896

- Heteroisotoma stebajevae (Rusek, 1991).

Troglophile, recorded from the Planovaya3 Cave in the Burzianskiy District of Bashkortostan, as well as from the entrance to the Druzhba Cave near Serga River, Sverdlovsk Region (Jie et al., 2011).

- Sericeotoma knissi Potapov, 1991. Troglobiont, described from the Yakshingulovskaya-1 Cave on the right bank of Belaya River in Bashkortostan (Potapov, 1991).

Superfamily Tomoceroidea Szeptycki, 1979

Family Tomoceridae Schäffer, 1896

- Plutomurus baschkiricus (Skorikow, 1899), troglobiont, endemic to many caves in Bashkortostan (Turbanov et al., 2016b), populations studied by Kniss (1984a).

- Tomocerus asiaticus Martynova, 1969 and four other troglophile Tomocerus spp. are recorded from the Urals.

All theses species are recent cave-dwellers which must have survived in caves during the glacial Pleistocene period (Kniss, Thibaud, 1999; Kniss, 1984a, 1984b, 1985, 2004).

Class Insecta Linnaeus, 1758

Order Psocoptera Shipley, 1904

Family Psyllipsocidae Lienhard et Smithers, 2002

- Psyllipsocus sp. Troglophile, recorded from caves of Bashkortostan, southern Urals (Kniss, 2001).

Order Coleoptera Linnaeus, 1758

Family Carabidae Latreille, 1802

- Bembidion obscurellum turanicum Csiki, 1928. Troglophile, recorded from caves of Bashkortostan, southern Urals (Kniss, 2001).

- Bembidion quadrimaculatum (Linnaeus, 1761). Mistakenly referred to as a troglophile, recorded from the Zapovednaya Cave, Beloretskiy District, Bashkortostan (Kniss, 2001), but actually, just like the previous subspecies, a trogloxene.

Family Cryptophagidae Kirby, 1937

- Micrambe nigricollis Reitter, 1876. Troglophile, recorded from the Shulgan-Tash Cave, Burzianskiy District, Bashkortostan (Kapralov, 2015). 
Table 3. List of *stygobionts, stygophiles and *troglobionts, troglophiles from the Urals. Таблица 3. Список *стигобионтов, стигофилов и *троглобиотов, троглофилов с Урала.

A - Stygobionts *: stygophiles

AMPHIPODA

Crangonyctidae

*Crangonyx chlebnikovi chlebnikovi Borutzky, 1928

B - Troglobionts *: troglophiles

Oligochaeta

Lumbricidae

Eiseniella tetraedra (Savigny, 1826)

Dendrobaena octaedra (Savigny, 1826)

Dendrodrilus rubidus (Savigny, 1826)

ARANEI

Linyphiidae

Improphantes improbulus (Simon, 1929)

Megalepthyphantes pseudocollinus Saaristo, 1997

Neriene montana (Clerck, 1758)

Nesticidae

Nesticus cellulanus (Clerck, 1758)

ACARI

Prostigmata: Eupodina: Rhagidiidae

* Rhagidia breviseta Zacharda, 1995

Rhagidia sp.

*Foveacheles sp.

Sarcoptiformes: Oribatida: Damaeidae

Epidamaeus sp.

Sarcoptiformes: Oribatida: Mochlozetidae

Sphaerobates sp.

ACARI PARASITI

Parasitidae

Parasitus oudemansi Berlese, 1903

Parasitus remberti (Oudemans, 1912)

Rhodacaridae

Cyrtolaelaps mucronatus Canestrini, 1881

CHILOPODA

Monotarsobius curtipes C.L. Koch, 1847

COLLEMBOLA

Dicyrtomidae

Dicyrtoma fusca (Lubbock, 1873)

Hypogastruridae

*Ceratophysella armata (Nicolet, 1842)

Ceratophysella bengtssoni (Agren, 1904)

Ceratophysella denticulata (Bagnall, 1941)

Ceratophysella kapoviensis (Babenko, 1994)

Ceratophysella sigillata (Uzel, 1890)

Ceratophysella succinea (Gisin, 1949)

Hypogastrura sahlbergi (Reuter, 1895)

Hypogastrura sensilis (Folsom, 1919)

Hypogastrura subboldorii Delamare et Jacquemin,

1962

Hypogastrura.vernalis (Carl, 1901)

*Schaefferia baschkirica Kniss, 1985

Typhlogastrura cf. alabamensis Thibaud, 1975

Entomobryidae

Entomobrya puncteola Uzel, 1891

Lepidocyrtus curvicollis (Bourlet, 1839)

Lepidocyrtus lanuginosus Gmelin, 1788

Lepidocyrtus violaceus (Fourcroy, 1785)

Isotomidae

Desoria olivacea (Tullberg, 1871)

Folsomia diplophthalma (Axelson, 1902)

Folsomia multiseta Stach, 1947

Folsomia quadrioculata Tullberg, 1871

Folsomia spinosa Kseneman, 1936

Heteroisotoma stebajevae Rusek, 1999

Isotoma albella Packard, 1873

Isotoma knissi Potapov, 1991

Isotoma viridis Bourlet, 1839

Proisotoma notabilis (Schaeffer, 1896)

Neanuridae

*Philotella olgae Kniss et Thibaud, 1996

Onychiuridae

Hymenaphorura sibirica (Tullberg, 1876)

Onychiuroides granulosus (Stach, 1930)

Onychiurus furcifer (Börner, 1901)

Onychiurus schoetti Lie-Pettersen, 1896

Protaphorura armata (Tullberg, 1869)

Tomoceridae

*Plutomurus baschkiricus (Skorikow, 1899)

Tomocerina minuta (Tullberg, 1876)

Tomocerus asiaticus Martynova, 1969

Tomocerus minor (Lubbock, 1862)

Tomocerus cf. punctatus Yosii, 1967

Tomocerus vulgaris (Tullberg, 1871)

Arrhopalitidae

Arrhopalites principalis Stach, 1945

PSOCOPTERA

Psyllipsocidae

Psyllipsocus sp.

COLEOPTERA

Carabidae

Bembidion obscurellum turanicum Csiki, 1928

Bembidion quadrimaculatum (Linnaeus, 1761)

Cryptophagidae

Micrambe nigricollis Reitter, 1876

Leiodidae

Choleva glauca (Britten, 1918)

Choleva lederiana Reitter, 1902

Staphylinidae

Atheta sp.

Falagria thoracica Curtis, 1833

Geostiba circellaris Gravenhorst, 1802 


\section{LEPIDOPTERA}

Noctuidae

Scoliopteryx libatrix (Linnaeus, 1758)

DIPTERA

Mycetophilidae

Speolepta leptogaster (Winnertz, 1863)

Sciaridae

Sciaridae gen. spp.

Trichoceridae

Trichocera maculipennis Meigen, 1818

Phoridae

Phoridae gen. spp.
Scathophagidae

Scathophagidae gen. spp.

CHIROPTERA

Vespertilionidae

Myotis dasycneme (Boie, 1825)

Myotis daubentonii (Kuhl, 1817)

Myotis nattereri (Kuhl, 1817)

Myotis brandtii (Eversmann, 1845)

Myotis mystacinus (Kuhl, 1817)

Myotis davidii Peters, 1869

Plecotus auritus (Linnaeus, 1758)

Eptesicus nilssonii (Keyserling et Blasius, 1839)
Family Leiodidae Fleming, 1821

- Choleva glauca (Britten, 1918). Subtroglophile, recorded from the Shulgan-Tash Cave, Burzianskiy District, Bashkortostan (Kapralov, 2015).

- Choleva lederiana Reitter, 1902. Subtroglophile, recorded from the Kungurskaya Ice Cave (Koz'minykh, 2012).

Family Staphylinidae Lameere, 1900

- Atheta sp. Troglophile, recorded from caves of the southern Urals: Ishcheevskaya2, Ishimbayskiy District, and Shulgan-Tash, Burzianskiy District, both Bashkortostan (Kniss, 2001).

- Falagria thoracica Curtis, 1833. Troglophile, recorded from the Bortovoy Otpor Cave and the Zilimskiy grotto, Gafuriyskiy District, Bashkortostan (Kniss, 2001).

- Geostiba circellaris Gravenhorst, 1802. Troglophile, recorded from caves of Bashkortostan, southern Urals (Kniss, 2001).

Order Lepidoptera Linnaeus, 1758

Family Noctuidae Latreille, 1809 - Scoliopteryx libatrix (Linnaeus, 1758). Troglophile, recorded from numerous caves in the Perm Province and Bashkortostan (Pan'kov et al., 2009b; Kapralov, 2015).

Order Diptera Linnaeus, 1758

Suborder Nematocera Schiner, 1862

Family Mycetophilidae Newman, 1835 - Speolepta leptogaster (Winnertz, 1863). Troglophile, recorded from the ShulganTash Cave, Burzianskiy District, Bashkortostan; the characteristic hunting nets also reported from the Dudkinskaya adit, Ufa City, and the Butylka Cave, both Bashkortostan as well (Kapralov, 2015).
Family Sciaridae Billberg, 1820

- Sciaridae gen. spp. Likely troglophiles, larvae encountered in several caves of European Russia, including the southern Urals. A reliable record concerns the Starosaitovskaya-1 Cave, Ishimbayskiy District, Bashkortostan (Kniss, 2001; Kapralov, 2015).

Family Trichoceridae Rondani, 1841

- Trichocera maculipennis Meigen, 1818. Troglophile, common in caves of the Perm Province (Pan'kov et al., 2009b; Kapralov, 2015).

Suborder Brachycera Zetterstedt, 1842

Family Phoridae Gray, 1840

- Phoridae gen. spp. Likely troglophiles, larvae encountered in several caves in the southern Urals, but remain unidentified as to genus and species (Kapralov, 2015).

Family Scathophagidae Robineau-Desvoidy, 1830

Specimens unidentified to genus and species have been encountered in the Babinogorskaya Cave, Kungurskiy District, Perm Province (Pan'kov et al., 2009b).

Phylum Chordata Haeckel, 1874

Subphylum Vertebrata J.-B. Lamarck, 1801

Class Mammalia Linnaeus, 1758

Order Chiroptera Blumenbach, 1779

Family Vespertilionidae Gray, 1821

According to recent data, eight bat species of the 14 occurring in the Urals are resident in the region, while others perform seasonal longdistance migrations and depart in winters. No maternity roosts have been found underground so far, as all records in caves were related to swarming or hibernating bats belonging to the 
following eight species (see Bol'shakov et al. (2005), Snit'ko (2007, 2009, 2011), and Snit'ko and Snit'ko (2016, 2017a, 2017b) for details):

- Myotis dasycneme (Boie, 1825) (Fig. 4A). This species, common in the region, was found in more than 50 cave hibernacula located from north to south along the Urals, with the largest known aggregations in the Smolinskaya and Arakaevskaya caves in the Sverdlovsk Region (up to 2,000 and over 200, respectively). In the southern Urals, no substantial winter colonies have been discovered, although this bat is relatively abundant at cave entrances during swarming time.

- Myotis daubentonii (Kuhl, 1817) (Fig. 4D). Daubenton's bat is as fairly common in the Urals as elsewhere within its distribution range in Russia; it was recorded in over 50 caves throughout the region. Unlike the Pond Bat, it forms no large winter aggregations. Therefore, it is usually undercounted during surveys at hibernation sites. The Smolinskaya and Arakaevskaya caves in the Sverdlovsk Region alongside the Nadezhda and Sukhokamenskaya caves in the Chelyabinsk Region, and the Kueshta Cave in Bashkiria comprise the largest winter colonies which range from 50 to 110 individuals.

- Myotis nattereri (Kuhl, 1817). The species was considered rare in the region because only few records from the middle and southern Urals were reported in the 20th century. However, Natterer's bats turned out to be very common in caves of the southern Urals in late summer and autumn, where these bats were caught in large numbers at cave entrances as they swarmed, with the largest number of 1,000 animals counted in the Kyzyl-Yar Cave in Bashkiria.

- Myotis brandtii (Eversmann, 1845) (Fig. 4E). Brandt's bat was found in $>20$ and 29 underground roosts in the middle and southern Urals, respectively. The Divya Cave in the Perm Province represents the northernmost hibernation roost in the Urals with the largest winter colony (up to 1,000 individuals); only a few Brandt's bats were found hibernating in other caves. Despite its relative rarity in winter, a number of Brandt's bats were netted at swarming sites in the southern Urals, where $M$. brandtii was the second most abundant species following $E$. nilssonii in Bashkiria and following P. auritus in the Chelyabinsk Region.

- Myotis mystacinus (Kuhl, 1817) (Fig. $4 \mathrm{~F})$. A few reliable records from the northern Urals were made in the Divya Cave. In the southern Urals, the species is abundant in swarming and hibernation undergrounds in Bashkiria, where about 50 bats were netted at the entrances to the caves Kueshta, Laklinskaya, Oktyabrskaya, Nadezhda and Sukhokamenskaya.

- Myotis davidii Peters, 1869. It is unclear yet, which of the previous records of bats from the M. mystacinus morpho-group should be assigned to this species (see Benda et al. (2016) for a taxonomic review). Two individuals identified as undoubtedly M. davidii were found in the Podarok Cave, Orenburg Region in September 2016.

- Plecotus auritus (Linnaeus, 1758) (Fig. 4B). The Brown long-eared bat is widely distributed, albeit not abundant in hibernation sites in the Urals. It was found in more than 60 caves throughout the region, northwards up to the Divya Cave. It was also very common in samples netted during swarming in the southern part of the region (Chelyabinsk Region and Bashkiria), where it constituted 15 and $24 \%$ of all captured bats, respectively.

- Eptesicus nilssonii (Keyserling et Blasius, 1839) (Fig. 4C). The Northern Bat is the most widely distributed and abundant species in the Urals. It has been found in more than 70 underground sites across the region, with the largest winter aggregation in the Arakaevskaya Cave, Perm Province. E. nilssonii comprised a large proportion of swarming bats at the entrances to caves in the southern Urals, being the most abundant species in the Chelyabinsk Region. 


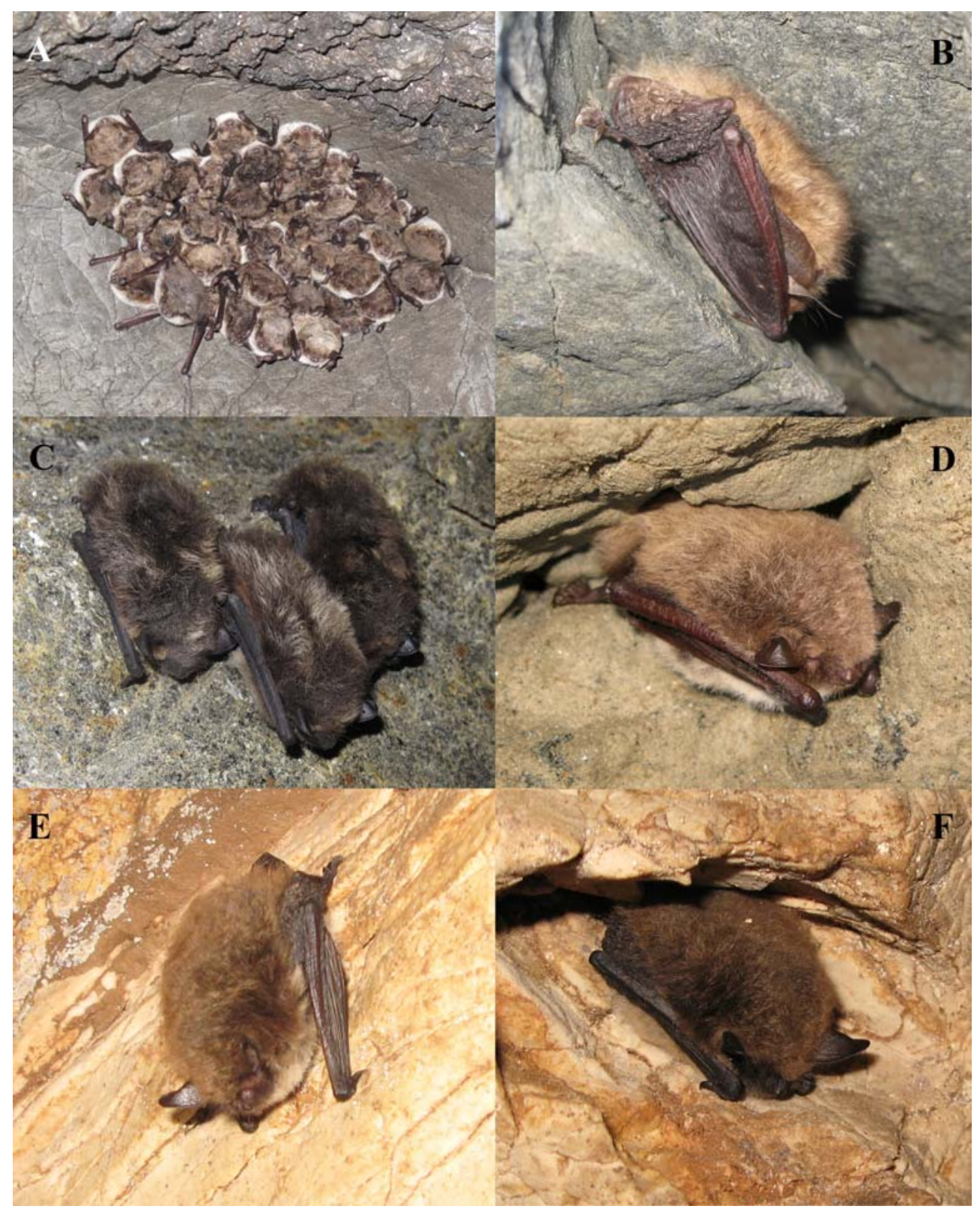

Fig. 4. Some species of bats during hibernation in caves of the Urals. A - hibernating colonies of the Pond bat, Myotis dasycneme (Boie, 1825) in the Smolinskaya Cave, Sverdlovsk Region. B - a hibernating Brown long-eared bat, Plecotus auritus (Linnaeus, 1758) in the Nadezhda Cave, Chelyabinsk Region. C - a hibernating Northern bat, Eptesicus nilssonii (Keyserling et Blasius, 1839) in the Nukatovskaya Cave, Bashkiria. D - a hibernating Daubenton's bat, Myotis daubentonii (Kuhl, 1819) in the Sukhaya Atya Cave, Chelyabinsk Region. E - a hibernating Brandtrs bat, Myotis brandtii (Eversmann, 1845) in the Kyzyl-Yar Cave, Bashkiria. F - a hibernating Whiskered bat, Myotis mystacinus (Kuhl, 1817) in the Sukhokamenskaya Cave, Chelyabinsk Region. All pictures by Ksenia Snit'ko. 
Conclusion. The subterranean fauna of the Urals, both terrestrial and aquatic, is mainly troglophile and stygophile, with a reduced number of endemic and relict cave species (Table 3). The impact of glacial Pleistocene phases may account for the scarcity of troglobionts and the relatively high number of recent troglophiles.

\section{4. The Russian Great Caucasus}

Charitonov (=Kharitonov) (1947), Malevich (1947), Borutzky (1934, 1948a, b, 1950a, b, 1961, 1965, 1967a, b), Birstein (1948, 1950, 1952, 1954, 1967), Starobogatov (1962), Ljovuschkin and Starobogatov (1963), Rudjakov (1963), Zalesskaja (1963, 1973a, b, 1978), Ljovuschkin and Matiokin (1965), Ljovuschkin (1962, 1963, 1965, 1966, 1970, 1972a, b, 1973), Birstein and Ljovuschkin (1967a), Danielopol (1969), Golovatch (1975, 1978, 1983, 19841985, 1990, 2011), Read (1992), Belousov (1999), Kniss and Thibaud (1999), Kantor and Sysoev (2005), Belousov and Koval (2009, 2011), Chemeris (2009), Karaman (2012), Antić and Makarov (2016) and several other authors have provided much of the information on invertebrates of the region in question. A full updated checklist and an as complete bibliography as possible concerning the entire territory of the former Soviet Union are available in Turbanov et al. (2016a, b, c).

The Russian Caucasus consists of Ciscaucasia (= Circassia) and the western part of Transcaucasia as far south as Psou River, bordering on Abkhazia. The subterranean biota can be estimated as being relatively well known in Russia, also being the richest in endemic and relict species with more than 80 troglo- and stygobionts revealed (Table 4).

Kingdom Chromista Cavalier-Smith, 1981 Phylum Ochrophyta Cavalier-Smith, 1995
Class Chrysophyceae Pascher, 1914 Order Ochromonadales Pascher, 1910

Family Paraphysomonadaceae Preisig et Hibberd, 1983

- Paraphysomonas bandaiensis (Hibberd, 1979). Likely stygophile, recorded from the Vorontsovskaya Cave, Khostinsky City District, Greater Sochi, Krasnodar Province (Myl'nikov et al., 2006).

Family Chromulinaceae Engler, 1897

- Spumella sp. Likely stygophile, recorded from the Vorontsovskaya Cave, Khostinsky City District, Greater Sochi, Krasnodar Province (Myl'nikov et al., 2006).

Class Raphidophyceae Chadefaud et Silva, 1980 Order Thaumatomonadida Shirkina, 1987

Family Thaumatomastigidae Patterson et Zolffel, 1991

- Thaumatomonas seravini Mylnikov and Karpov, 1993. Likely stygophile, recorded from the Vorontsovskaya Cave, Khostinsky City District, Greater Sochi, Krasnodar Province (Myl'nikov et al., 2006).

Superphylum Alveolata Cavalier-Smith, 1991 Phylum Ciliophora Doflein, 1901

Class Phyllopharyngea de Puytorac et al., 1974 Subclass Suctoria Claparède et Lachmann, 1858

- Suctoria gen. sp. Epibiont forms found on hypogean amphipods in the Beloskal'skaya and Pionerskaya (= Ushchel'naya) caves, Khostinsky City District, Greater Sochi, Krasnodar Province (Birstein, 1950).

Kingdom Rhizaria Cavalier-Smith, 2002 Phylum Cercozoa Cavalier-Smith, 1998

Class Sarcomonadea Cavalier-Smith, 1993

Order Glissomonadida Howe, Bass, Vickerman, Chao et Cavalier-Smith, 2009

Family Allapsidae Howe, Bass, Vickerman, Chao et Cavalier-Smith, 2009

Рис. 4. Некоторые виды летучих мышей во время зимовки в пещерах Урала. А - зимующие колонии ночницы прудовой (Myotis dasycneme (Boie, 1825)) в Смолинской пещере (Свердловская обл.). В зимующий ушан (Plecotus auritus (Linnaeus, 1758)) в пещере Надежда (Челябинская обл.). С зимующий северный кожанок (Eptesicus nilssonii (Keyserling et Blasius, 1839)) в пещере Нукатовская (Башкирия). D - зимующая водяная ночница (Myotis daubentonii (Kuhl, 1819)) в пешере Сухая Атья (Челябинская обл.). Е - зимующая ночница Брандта (Myotis brandtii (Eversmann, 1845)) в пещере Кызыл-Яр (Башкирия). F — зимующая усатая ночница (Myotis mystacinus (Kuhl, 1817)) в пещере Сухокаменская (Челябинская обл.). Все фотографии Ксении Снитько. 
Table 4. List of *stygobionts, possible stygobionts, stygophiles, possible troglobionts and troglophiles from the Caucasus. Таблица 4. Список *стигобионтов, вероятных стигобионтов, стигофилов, возможных троглобиотов и троглофилов с Кавказа.

A - Stygobionts *: stygophiles

\section{CHROMISTA}

Paraphysomonadaceae

Paraphysomonas bandaiensis (Hibberd, 1979)

Chromulinaceae

Spumella sp.

RHIZARIA

Thaumatomastigidae

Thaumatomonas seravini Mylnikov et Karpov, 1993

Thaumatomonas seravini Mylnikov et Karpov, 1993

Allapsidae

Allantion tachyploon Sandon, 1924

Heteromitidae

Heteromita globosa (Stein, 1878)

Heteromita sp.

ALVEOLATA

Suctoria gen. sp.

MYZOZOA

Protaspidaceae

Protaspis simplex Vors, 1992

Colponemidae

Colponema edaphicum Mylnikov et Tikhonenkov, 2007

\section{SARCOMASTIGOTA}

Bodonaceae

Bodo designis Skuja, 1948

Bodo saliens Larsen et Paterson, 1990

Bodo saltans Ehrenberg, 1838

Cercomonas granulifera (Hollande, 1942)

Cercomonas laciniaegerens (Krassilstschick, 1886)

Cercomonas sp.

Dimastigella mimosa Frolov, Mylnikov et Malysheva, 1997

Rhynchomonas nasuta (Stokes) Klebs, 1892

AMOEBOZOA

Centropyxidae

Centropyxis constricta (Ehrenberg, 1841)

Centropyxis ecornis (Ehrenberg, 1841)

Centropyxis orbicularis Deflandre, 1929

Centropyxis plagiostoma Bonnet et Thomas, 1955

Centropyxis platystoma (Penard, 1890)

Centropyxis spinosa Cash, 1905

Difflugiidae

Difflugia avellana Penard, 1890

Difflugia avellana gigas Gauthier-Lievre et Thomas, 1958

Difflugia penardi Hopkinson, 1909

Difflugia oblonga Ehrenberg, 1838

Pontigulasia incisa Rhumbler, 1896

Heleoperidae

Heleopera sphagni Leidy, 1874

MYCETOZOA
Physaridae

Physarum flagellatum (Alexeieff, 1923)

PLATYHELMINTHES

Tricladida gen. sp.

Dugesiidae

Dugesia taurocaucasica (Livanov, 1951)

Dendrocoelidae

Dendrocoelidae gen. sp.

*Dendrocoelum sp. 1

*Dendrocoelum sp. 2

MOLLUSCA: GASTROPODA

Hydrobiidae

Belgrandiella caucasica Starobogatov, 1962

*"Geyeria" valvataeformis Starobogatov, 1962

*"Lartetia" sp.

*Paladilhiopsis orientalis Starobogatov, 1962

*Paladilhiopsis pulcherrima Starobogatov, 1962

*Paladilhiopsis subovata Starobogatov, 1962

MOLLUSCA: BIVALVIA

Euglesidae

Euglesa personata (Malm, 1855)

*Euglesa cavatica (Shadin, 1952)

HIRUDINEA

Erpobdellidae

Erpobdella octoculata (Linnaeus, 1758)

COPEPODA

Cyclopoida

Cyclopidae

*Acanthocyclops venustus stammeri (Kiefer, 1930)

Diacyclops bisetosus (Rehberg, 1880)

Paracyclops fimbriatus imminutus Kiefer, 1929

*Speocyclops cinctus Monchenko, 1982

*Speocyclops lussianus Borutzky, 1950

*Speocyclops psezuapsensis Borutzky, 1965

Harpacticoida

Ameiridae

*Nitocrella hirta caucasica Borutzky, 1967

*Megastygonitocrella ljovuschkini (Borutzky, 1967)

Canthocamptidae

Attheyella crassa (G.O. Sars, 1863)

*Bryocamptus aquaeductus Borutzky, 1940

Bryocamptus hostensis Borutzky, 1972

*Bryocamptus innominatus Borutzky, 1940

Bryocamptus pygmaeus (G.O. Sars, 1863)

Bryocamptus tarnogradskyi Borutzky, 1934

Bryocamptus zschokkei caucasicus Borutzky, 1930

Canthocamptus staphylinus (Jurine 1820)

Elaphoidella bidens coronata (G.O. Sars, 1904)

*Pilocamptus georgevitchi (Chappuis, 1923)

*Elaphoidella czerkessica Borutzky, 1972

*Moraria hostensis Borutzky, 1972 
* Moraria operculata Borutzky, 1948

*Moraria poppei (Mrazek, 1893)

*Moraria varica (Graeter, 1910)

Parastenodarididae

*Parastenocaris tenuis Borutzky, 1948

*Parastenocaris sp.

Ostracoda

Candonidae

*Trapezicandona ljovuschkini (Rudjakov, 1963)

Cyprididae

Cypria reptans Bronstein, 1947

Limnocytheridae

*Kovalevskiella rudjakovi (Danielopol, 1969)

Syncarida

Bathynellidae

*Antrobathynella stammeri ciscaucasica (Birstein et Ljovuschkin, 1964)

*Bathynella natans natans Vejdovsky, 1882

Isopoda

Asellota: Asellidae

*Proasellus linearis (Birstein, 1967)

*Proasellus ljovuschkini (Birstein, 1967)

*Proasellus similis (Birstein, 1967)

B - Troglobionts *: troglophiles

\section{OLIGOCHETA}

Lumbricidae

Allolobophora cavatica Michaelsen, 1910

Dendrobaena veneta crassa (Malevics, 1947)

Eisenia fetida (Savigny, 1826)

Naididae

Homochaeta sp.

Peloscolex sp.

NEMATODA

Monhysteridae

Monchystera paludicola De Man, 1880

Dorylaimidae

Dorylaimus stagnalis Dujardin, 1845

Dorylaimus callosus Skwarra, 1921

Eudorylaimus laticollis (De Man, 1907)

Eudorylaimus condamni (Vanha, 1893)

Eudorylaimus iners (Bastian, 1865)

Actinolaimidae

Trachactinolaimus radulatus Andrássy, 1963

Mononchidae

Mononchus truncatus Bastian, 1865

Prionchulus muscorum (Dujardin, 1845)

Tripylidae

Tripyla papillata Bütschli, 1873

Tripyla filicaudata De Man, 1880

Prismatolaimidae

Prismatolaimus dolichurus De Man, 1880

Criconematidae

Criconema fimbriatum Cobb in Taylor, 1936

Criconema sp.

Criconemoides sp.
Asellota: Microparasellidae

*Microcharon tantalus Birstein et Ljovuschkin, 1964

AMPHIPODA

Crangonyctidae

*Lyurella shepsiensis Sidorov, 2015

Niphargidae

*Niphargus abchasicus Martynov, 1932

*Niphargus caelestis G. Karaman, 1982

*Niphargus cubanicus Birstein, 1954

*Niphargus krasnodarus G. Karaman, 2012

*Niphargus latimanus Birstein, 1952

*Niphargus cf. magnus Birstein, 1940

*Niphargus potamophilus Birstein, 1954

*Niphargus pseudolatimanus Birstein, 1952

*Niphargus smirnovi Birstein, 1952

*Niphargus submersus (Derzhavin, 1945)

Typhlogammaridae

*Zenkevitchia $\mathrm{sp}$.

DECAPODA

Caridea: Atyidae

*Troglocaris (Xyphocaridella) jusbaschjani Birstein, 1948

Brachyura: Potamidae

Potamon ibericum tauricum (Czerniavsky 1884)

Mollusca

Orculidae

Lauria sp.

Trigonochlamydidae

*Troglolestes sokolovi Ljovuschkin et Matiokin, 1965

Orculidae

*Euxinolauria vitrea (Schileyko, 1988)

Clausiliidae

Scrobifera taurica (L. Pfeiffer, 1848)

Serrulina sp.

Zonitidae

*Conulopolita cavatica (Riedel, 1966)

Oxychilus (Longiphallus) sp.

ISOPODA TERRESTRIA

Oniscidea

Ligiidae

Ligidium zaitzevi Borutzky, 1950

*Ligidium cavaticum Borutzky, 1950

Buddelundiellidae

*Pseudobuddelundiella hostensis Borutzky, 1967

*Pseudobuddelundiella ljovuschkini Borutzky, 1967

*Trichoniscus pygmaeus tuapsensis Borutzky, 1972

Cylisticidae

Cylisticus birsteini Borutzky, 1961

Trichoniscidae

*Caucasocyphonethes cavaticus Borutzky, 1948

*Psachonethes czerkessicus Borutzky, 1969

ARANEI

Agelenidae

Tegenaria abchasica Charitonov, 1941 
Tegenaria pontica Charitonov, 1947

Tegenaria sp.

Hahniidae

Iberina ljovuschkini Pichka, 1965

Tetragnathidae

Meta bourneti Simon, 1922.

Metellina merianae (Scopoli, 1763)

Linyphiidae

Troglohyphantes birsteini Charitonov, 1943

Nesticidae

Carpathonesticus birsteini (Charitonov, 1947)

Carpathonesticus ljovuschkini (Pichka, 1965)

Carpathonesticus zaitzevi (Charitonov, 1939)

Carpathonesticus sp.

Aituaria pontica (Spassky, 1932)

Pholcidae

Hoplopholcus longipes (Spassky, 1934)

PSEUDOSCORPIONES

Neobisiidae

*Neobisium (Neobisium) speleophilum Krumpal, 1986

*Neobisium sp.

OPILIONES

Nemastomatidae

Histricostoma caucasicum (Redikorzev 1936)

*Nemaspela abchasica (Ljovuschkin et Starobogatov, 1963)

*Nemaspela sokolovi (Ljovuschkin et Starobogatov, 1963)

*Nemaspela kovalii Chemeris, 2009

Phalangiidae

Nelima pontica Charitonov, 1941

ACARI

Phthiracaridae

Phthiracarus globosus (C.L. Koch, 1841)

Ixodidae

Ixodes vespertilionis C.L. Koch, 1844

CHILOPODA

Lithobiidae

Harpolithobius perplexus Zalesskaja, 1973

Lithobius liber Lignau, 1903

Lithobius reconditus Zalesskaja, 1973

Lithobius stuxbergii Seliwanoff, 1880

DIPLOPODA

Glomeridellidae

Typhloglomeris caucasica Golovatch, 1975

Doderiidae

Trachysphaera costata (Waga, 1857)

Trachysphaera minuta Golovatch, 1976

Anthroleucosomatidae

*Caucaseuma elephantum Antić et Makarov, 2016

*Cancaseuma fanagoriyskaya Antić et Makarov, 2016

*Cancaseuma lohmanderi Strasser, 1970

*Caucaseuma minellii Antić et Makarov, 2016

*Heterocancaseuma feminaepectorum Antić et Makarov, 2016

Metamastigophorophyllon giljarovi (Lang, 1959)
Blaniulidae

*Nopoiulus ammonites Enghoff, 1984

Nopoiulus kochii (Gervais, 1847)

Julidae

Archileucogeorgia sp.

Cylindroiulus placidus (Lignau, 1903)

Cylindroiulus pterophylacum Read, 1992

Cylindroiulus schestoperovi Lohmander, 1932

Paradoxosomatidae

Strongylosoma kordylamythrum Attems, 1898

Polydesmidae

Brachydesmus furcatus Lohmander, 1936

Trichopolydesmidae

*Caucasodesmus inexpectatus Golovatch, 1985

Collembola

Hypogastruridae

*Typhlogastrura preobrazhenskyi Babenko, 1987

Isotomidae

Desoria fennica (Reuter, 1895)

Tomoceridae

*Plutomurus jelesnovodskii Kniss et Thibaud, 1999

*Plutomurus kelasuricus (Martynova, 1969)

*Plutomurus sorosii Kniss et Thibaud, 1999

ORTHOPTERA

Rhaphidophoridae

*Dolichopoda euxina Semenov, 1901

TRICHOPTERA

Polycentropodidae

Plectrocnemia sp. sensu Lepneva, 1940

COLEOPTERA

Carabidae

*Caucasaphaenops molchanovi Belousov, 1999

*Caucasorites kovali amplicolis Belousov, 1999

*Caucasorites kovali Belousov, 1999

*Caucasorites shchurovi Belousov et Zamotajlov, 1997

*Caucasorites victori Belousov, 1999

Cimmerites circassicus Reitter, 1888

*Cimmerites kryzhanovskii Belousov 1999

*Cimmerites maximovitchi Belousov et Koval, 2011

Cimmerites zamotajlovi Belousov, 1998

*Duvalius miroshnikovi Belousov et Zamotajlov, 1995

Duvalius gusevi Belousov, 1989

*Jeannelius birsteini Ljovuschkin, 1965

Jeannelius zhicharevi Lutshnik, 1915

Laemostenus koenigi (Reitter, 1887)

Laemostenus tschitscherini Semenov, 1909

Meganophthalmus irinae Belousov et Zamotajlov, 1999

*Meganophthalmus kravetzi Komarov, 1993

*Nannotrechus ciscaucasiens (Ljovuschkin, 1972)

Nannotrechus fishtensis Belousov, 1989

Nannotrechus kovali Belousov, 1989

Porocimmerites imitator Belousov, 1998

Pterostichus lacunosus (Chaudoir 1844)

Trechus heniochicus Ljovuschkin, 1970 
Cryptophagidae

Cryptophagus pilosus Gyllenhal, 1827

Curculionidae

*Otiorhynchus vargovitchi Davidian, 2007

Elmidae

Limnius colchicus Delève, 1963

Leiodidae

Catops subfuscus Kellner, 1846

Choleva sp.

Pselaphidae

Bryaxis balneator Besuchet et Kurbatov, 2007

Bryaxis kovali Besuchet et Kurbatov, 2007

*Seracamaurops komarovi Hlaváč, Kodada et Koval, 1999

Ptiliidae

Ptenidium intermedium Wankowicz, 1896

Scirtidae

Odeles sp.

Staphylinidae

Bisnius parcus (Sharp, 1874)

Heinzia caucasica Gusarov et Koval, 2002

DIPTERA

Limoniidae

Limonia nubeculosa Meigen, 1804

Sciaridae

Neosciara sp.

Heleomyzidae

Heteromyza atricornis Meigen, 1830

\section{CHIROPTERA}

Rhinolophidae

Rhinolophus euryale Blasius, 1853

Rhinolophus ferrumequinum (Schreber, 1774)

Rhinolophus hipposideros (Borkhausen, 1797)

Rhinolophus mehelyi Matschie, 1901

Vespertilionidae

Barbastella barbastellus (Schreber, 1774)

Barbastella caspica Satunin, 1908

Eptesicus serotinus (Schreber, 1774)

Hypsugo savii (Bonaparte, 1837)

Myotis alcathoe von Helversen et Heller, 2001

Myotis davidii Peters, 1869

Myotis bechsteinii (Kuhl, 1817)

Myotis blythii (Tomes, 1857)

Myotis brandtii (Eversmann, 1845)

Myotis dasycneme (Boie, 1825)

Myotis daubentonii (Kuhl, 1817)

Myotis emarginatus (Geoffroy, 1806)

Myotis mystacinus (Kuhl, 1817)

Myotis nattereri (Kuhl, 1817)

Nyctalus noctula (Schreber, 1774)

Pipistrellus pipistrellus (Schreber, 1774)

Plecotus auritus (Linnaeus, 1758)

Plecotus macrobullaris Kuzyakin, 1965

Miniopteridae

Miniopterus schreibersii (Kuhl, 1817)

Molossidae

Tadarida teniotis (Rafinesque, 1814)
- Allantion tachyploon Sandon, 1924. Likely stygophile, recorded from the Vorontsovskaya Cave, Khostinsky City District, Greater Sochi, Krasnodar Province (Myl'nikov et al., 2006).

Order Cercomonadida Poche, 1913

Family Heteromitidae Kent, 1880

- Heteromita globosa (Stein, 1878). Likely stygophile, recorded from the Vorontsovskaya Cave, Khostinsky City District, Greater Sochi, Krasnodar Province (Myl'nikov et al., 2006).

- Heteromita sp. Likely stygophile, recorded from the Vorontsovskaya Cave, Khostinsky City District, Greater Sochi, Krasnodar Province (Myl'nikov et al., 2006).

Phylum Myzozoa Cavalier-Smith et Chao, 2004

Class Dinophyceae Fritsch, 1927

Order Desmomastigales Bourrelly, 1970

Family Protaspidaceae Skuja, 1939

- Protaspis simplex Vors, 1992. Likely stygophile, recorded from the Vorontsovskaya Cave, Khostinsky City District,
Greater Sochi, Krasnodar Province (Myl'nikov et al., 2006).

Class Colponemea Cavalier-Smith, 1993

Order Colponemida Cavalier-Smith, 1993

Family Colponemidae Cavalier-Smith et Chao, 2004

- Colponema edaphicum Mylnikov et Tikhonenkov, 2007. Likely stygophile, described from the Vorontsovskaya Cave, Khostinsky City District, Greater Sochi, Krasnodar Province(Myl'nikov et al., 2006; Myl'nikov, Tikhonenkov, 2007).

Kingdom Protozoa R. Owen, 1858

Subkingdom Sarcomastigota Cavalier-Smith, 1983

Group Excavata (Cavalier-Smith), 2002

Phylum Euglenozoa Cavalier-Smith, 1981

Class Kinetoplastea Honigberg, 1963

Order Eubodonida Vickerman, in Moreira et al., 2004

Family Bodonaceae Bütschli, 1884

- Bodo designis Skuja, 1948. Likely stygophile, recorded from the Vorontsovskaya 
Cave, Khostinsky City District, Greater Sochi, Krasnodar Province (Myl'nikov et al., 2006).

- Bodo saliens Larsen et Paterson, 1990. Likely stygophile, recorded from the Vorontsovskaya Cave, Khostinsky City District, Greater Sochi, Krasnodar Province (Myl'nikov et al., 2006).

- Bodo saltans Ehrenberg, 1838. Likely stygophile, recorded from the Vorontsovskaya Cave, Khostinsky City District, Greater Sochi, Krasnodar Province (Myl'nikov et al., 2006).

- Cercomonas granulifera (Hollande, 1942). Likely stygophile, recorded from the Vorontsovskaya Cave, Khostinsky City District, Greater Sochi, Krasnodar Province (Myl'nikov et al., 2006).

- Cercomonas laciniaegerens (Krassilstschick, 1886). Likely stygophile, recorded from the Vorontsovskaya Cave, Khostinsky City District, Greater Sochi, Krasnodar Province (Myl'nikov et al., 2006).

- Cercomonas sp. Likely stygophile, recorded from the Vorontsovskaya Cave, Khostinsky City District, Greater Sochi, Krasnodar Province (Myl'nikov et al., 2006).

- Dimastigella mimosa Frolov, Myl'nikov et Malysheva, 1997. Likely stygophile, recorded from the Vorontsovskaya Cave, Khostinsky City District, Greater Sochi, Krasnodar Province(Myl'nikov et al., 2006). - Rhynchomonas nasuta (Stokes) Klebs, 1892. Likely stygophile, recorded from the Vorontsovskaya Cave, Khostinsky City District, Greater Sochi, Krasnodar Province (Myl'nikov et al., 2006).

Phylum Amoebozoa Lühe, 1913

Class Tubulinea Smirnov, Nassonova, Berney,

Fahrni, Bolivar et Pawlowski, 2005

Order Arcellinida Kent, 1880

Family Centropyxidae Jung, 1942

- Centropyxis constricta (Ehrenberg, 1841). Stygophile, recorded from the Labirintovaya Cave, Khostinsky City District, Greater Sochi, Krasnodar Province (Chibisova, 1967).
- Centropyxis ecornis (Ehrenberg, 1841). Likely stygophile, recorded from sulfuric springs in the karst canyon of Agura River, Khostinsky City District, Greater Sochi, Krasnodar Province (Chibisova, 1967).

- Centropyxis orbicularis Deflandre, 1929. Stygophile, recorded from the Labirintovaya Cave, Khostinsky City District, Greater Sochi, Krasnodar Province (Chibisova, 1967).

- Centropyxis plagiostoma Bonnet et Thomas, 1955. Stygophile, recorded from the Labirintovaya Cave, Khostinsky City District, Greater Sochi, Krasnodar Province (Chibisova, 1967; Ljovuschkin 1972b).

- Centropyxis platystoma (Penard, 1890). Stygophile, recorded from the Labirintovaya Cave, Khostinsky City District, Greater Sochi, Krasnodar Province (Chibisova, 1967).

- Centropyxis spinosa Cash, 1905. Stygophile, recorded from the Labirintovaya Cave, Khostinsky City District, Greater Sochi, Krasnodar Province (Chibisova, 1967).

Family Difflugiidae Wallich, 1864

- Difflugia avellana Penard, 1890. Stygophile, recorded from the Labirintovaya Cave, Khostinsky City District, Greater Sochi, Krasnodar Province (Chibisova, 1967).

- Difflugia avellana gigas Gauthier-Lievre et Thomas, 1958. Stygophile, recorded from the Pionerskaya (= Ushchel'naya) Cave, Khostinsky City District, Greater Sochi, Krasnodar Province (Chibisova, 1967).

- Difflugia penardi Hopkinson, 1909. Stygophile, recorded from the Labirintovaya Cave, Khostinsky City District, Greater Sochi, Krasnodar Province (Chibisova, 1967).

- Difflugia oblonga Ehrenberg, 1838. Stygophile, recorded from the Labirintovaya Cave, Khostinsky City District, Greater Sochi, Krasnodar Province (Chibisova, 1967).

- Pontigulasia incisa Rhumbler, 1896. Stygophile, recorded from the Labirintovaya 
Cave, Khostinsky City District, Greater Sochi, Krasnodar Province (Chibisova, 1967).

Family Heleoperidae Jung, 1942

- Heleopera sphagni Leidy, 1874 (= Heleopera picta Leidy, 1879). Likely stygophile, recorded from the Labirintovaya Cave, Khostinsky City District, Greater Sochi, Krasnodar Province (Chibisova, 1967).

Subphylum Conosa Cavalier-Smith, 1998

Infraphylum Mycetozoa de Bary, 1859

Class Myxogastria Macbride, 1899

Order Physarida Macbride, 1922

Family Physaridae Rostafinski, 1873

- Physarum flagellatum (Alexeieff, 1923) (= Hyperamoeba flagellata Alexeieff, 1923). Likely stygophile, recorded from the Labirintovaya Cave, Khosta District, Greater Sochi, Krasnodar Province (Myl'nikov et al., 2006).

Kingdom Animalia Linnaeus, 1758

Phylum Platyhelminthes Claus, 1887

Class Turbellaria Ehrenberg, 1831

Order Tricladida Lang, 1884

Unidentified unpigmented planarians have long been recorded from the Bolshaya Vorontsovskaya (= Vorontsovskaya)Cave, Khostinsky City District, Greater Sochi, Krasnodar Province (Birstein, 1950). Recorded from a tectonic cave on the Psebe River, Tuapse District, as well as the Kirovskaya (Lazarevsky City District, Greater Sochi) and Labirintovaya caves (Khostinsky City District, Greater Sochi), all in Krasnodar Province (Ljovuschkin, 1966).

Family Dugesiidae Ball, 1974

- Dugesia taurocaucasica (Livanov, 1951). Stygophile, recorded from a cave on Mt Armovka, Tryu-Yatyrgvarta Massif, Mostovskoy District, as well as from the Fanagoriyskaya Cave near Goryachiy Klyuch, both Krasnodar Province (Shumeev, 2008).

Family Dendrocoelidae Hallez, 1892

- Dendrocoelidae gen. sp. Likely stygobiont, closer unidentified, recorded from a spring inside the Anglo-Russkaya Cave on Mt Pshekha-Su, Maikopsky District, Adygea Republic (Shumeev, 2008).
- Dendrocoelum sp. 1. Stygophile, an undescribed species from the running waters of the Tryu-Yatyrgvarta Massif, including a spring inside a cave on Mt Armovka, Mostovskoy District, Krasnodar Province (Shumeev, 2008).

- Dendrocoelum sp. 2. Stygobiont, an undescribed species from the running waters inside the Fanagoriyskaya Cave near Goryachiy Klyuch, Krasnodar Province (Shumeev, 2008).

Phylum Nematoda Diesing, 1861

Uncertain species of nematodes have been recorded from the Pionerskaya (= Ushchel'naya) Cave, Khostinsky City District, Greater Sochi, Krasnodar Province (Ljovuschkin, 1966).

Class Enoplea Inglis, 1983

Order Monhysterida Filipjev, 1929

Family Monhysteridae De Man, 1876 - Monchystera paludicola De Man, 1880. Troglophile, recorded from the Labirintovaya (= Novaya) Cave, Greater Sochi, Krasnodar Province (Ljovuschkin, 1966).

Family Actinolaimidae Thorne, 1939

- Trachactinolaimus radulatus Andrássy, 1963. Troglophile, recorded from the Tonnel Kuzmenko Cave, Khostinsky City District, Greater Sochi, Krasnodar Province (Ljovuschkin, 1966).

Family Dorylaimidae De Man, 1876

- Dorylaimus stagnalis Dujardin, 1845. Troglophile, recorded from the Bolshaya Kazachebrodskaya Cave, Adlersky City District, Greater Sochi, Krasnodar Province (Ljovuschkin, 1966).

- Dorylaimus callosus Skwarra, 1921. Troglophile, recorded from the karst waters on the Agura River, Greater Sochi, Krasnodar Province (Ljovuschkin, 1966).

- Eudorylaimus laticollis (De Man, 1907). Troglophile, recorded from the Labirintovaya (= Novaya) Cave, Greater Sochi, Krasnodar Province (Ljovuschkin, 1966). - Eudorylaimus condamni (Vanha, 1893). Troglophile, recorded from the Dolgaya Cave, Khostinsky City District, Greater Sochi, Krasnodar Province (Ljovuschkin, 1966). 
- Eudorylaimus iners (Bastian, 1865). Troglophile, recorded from the Vorontsovskaya Cave, Khostinsky City District, Greater Sochi, Krasnodar Province (Ljovuschkin, 1966).

Order Mononchida Jairajpuri, 1969

Family Mononchidae Chitwood et Chitwood, 1937

- Mononchus truncatus Bastian, 1865. Troglophile, recorded from the Zapovednaya (= Podparapetnaya) Cave, Khostinsky City District, Greater Sochi, Krasnodar Province (Ljovuschkin, 1966).

- Prionchulus muscorum (Dujardin, 1845). Troglophile, recorded from the Dolgaya Cave, Khostinsky City District, Greater Sochi, Krasnodar Province (Ljovuschkin, 1966).

Order Triplonchida Cobb, 1920

Family Tripylidae (De Man, 1876)

- Tripyla papillata Bütschli, 1873. Troglophile, recorded from the karst waters on the Agura River, Greater Sochi, Krasnodar Province (Ljovuschkin, 1966).

- Tripyla filicaudata De Man, 1880. Troglophile, recorded from the Krasnoaleksandrovskaya, Labirintovaya (= Novaya) and Bolshaya Kazachebrodskaya caves, Greater Sochi, Krasnodar Province (Ljovuschkin, 1966).

Family Prismatolaimidae Micoletzky, 1922 - Prismatolaimus dolichurus De Man, 1880. Troglophile, recorded from the Labirintovaya (= Novaya) Cave, Greater Sochi, Krasnodar Province (Ljovuschkin, 1966).

Class Secernentea Lorenzen, 1981

Order Tylenchida Thorne, 1949

Family Criconematidae Taylor, 1936

- Criconema fimbriatum Cobb in Taylor, 1936. Troglophile, recorded from the Krasnoaleksandrovskaya and Pionerskaya (= Ushchel'naya) caves, Greater Sochi, Krasnodar Province (Ljovuschkin, 1966). - Criconema sp. Troglophile, recorded from the Fanagoriyskaya Cave near Goryachiy Klyuch, Krasnodar Province (Ljovuschkin, 1966).

- Criconemoides sp. Troglophile, recorded from the Labirintovaya (= Novaya) Cave,
Greater Sochi, Krasnodar Province (Ljovuschkin, 1966).

Phylum Annelida Lamarck, 1809

Class Hirudinea Lamarck, 1818

Order Arhynchobdellida Blanchard, 1894

Family Erpobdellidae Blanchard, 1894

- Erpobdella octoculata (Linnaeus, 1758), trogloxene, recorded by Birstein and Ljovuschkin (1967a) from the Fanagoriyskaya Cave near Goryachiy Klyuch, Krasnodar Province; also reported from several Balkan caves (Montenegro, Republic of Macedonia, Greek Macedonia).

Class Oligochaeta Grube, 1850

Order Crassiclitellata Jamieson, 1988

Family Lumbricidae Claus, 1876

- Allolobophora cavatica Michaelsen, 1910. Troglophile, described from a cave at the Khodz' River, Adygea Republic or Krasnodar Province. Also recorded from epigean habitats in Transcaucasia (Lenkoran, Azerbaijan) (Michaelsen, 1910).

- Dendrobaena veneta crassa (Malevics, 1947). Troglophile, described as a separate form from the Vorontsovskaya Cave, Khostinsky City District, Greater Sochi, Krasnodar Province (Malevich, 1947).

- Eisenia foetida (Savigny, 1826). Troglophile, recorded from the Podzemnaya Khosta (in the publication of Malevich, it was referred to as Khostinskaya Mokraya) and Bolshaya Kazachebrodskaya (= VerkhneMzymtinskaya, Mzymtinskaya) caves, both in Greater Sochi, Krasnodar Province (Malevich, 1947).

Order Enchytraeida Vejdovský, 1879

Family Enchytraeidae Vejdovský, 1879

Uncertain enchytraeid species have been recorded from the Fanagoriyskaya, Zapovednaya (= Podparapetnaya), Labirintovaya (= Novaya) and Vorontsovskaya caves, all Krasnodar Province (Ljovuschkin, 1966).

Order Tubificida Brinkhurst, 1982

Family Naididae Ehrenberg, 1828

- Homochaeta sp. Troglophile, recorded from the Zapovednaya (= Podparapetnaya) Cave, Khostinsky City District, Greater 
Sochi, Krasnodar Province (Ljovuschkin, 1966).

Peloscolex sp. Troglophile, recorded from the Labirintovaya (= Novaya) Cave, Greater Sochi, Krasnodar Province (Ljovuschkin, 1966).

Phylum Mollusca Linnaeus, 1758

Class Gastropoda Cuvier, 1797

Order Littorinimorpha Golikov et Starobogatov, 1975

Family Hydrobiidae Stimpson, 1865

- Belgrandiella caucasica Starobogatov, 1962 (= B. nemethi Schütt in Schütt et Şeşen, 1993). Stygo- and crenobiont, described from a spring inside the Krasnoaleksandrovskaya (= Peshchera Ved'm) Cave, Lazarevsky City District, Greater Sochi, Krasnodar Province (Starobogatov, 1962). Inhabiting cave (e.g. the Dolgaya Cave) and creek waters along the Black Sea coast between Tuapse and Psou (Starobogatov, 1962; Schütt, Şeşen, 1993; Palatov, Vinarski, 2015; Vinarski, Kantor, 2016).

- "Geyeria" valvataeformis Starobogatov, 1962. Stygobiont, known only from the type locality, a spring inside the Krasnoaleksandrovskaya (= Peshchera Ved'm) Cave, Lazarevsky City District, Greater Sochi, Krasnodar Province (Starobogatov, 1962; Vinarski, Kantor, 2016).

- "Lartetia" sp. Recorded from the Bolshaya Kazachebrodskaya (= Verkhne-Mzymtinskaya) Cave, Adlersky City District, Greater Sochi, Krasnodar Province (Birstein, 1950), but actually it seems to concern one of the Transcaucasian species of Paladilhiopsis (Turbanov et al., 2016a).

- Paladilhiopsis orientalis Starobogatov, 1962. Stygobiont, known only from the type locality, a spring inside the Krasnoaleksandrovskaya (= Peshchera Ved'm) Cave, Lazarevsky City District, Greater Sochi, Krasnodar Province (Starobogatov, 1962; Vinarski, Kantor, 2016).

- Paladilhiopsis pulcherrima Starobogatov, 1962. Stygo- and crenobiont, described from a spring inside the Krasnoaleksandrovskaya (= Peshchera Ved'm) Cave, Laz- arevsky City District, Greater Sochi, Krasnodar Province (Starobogatov, 1962). Inhabiting also helo- and rheocrens along the Black Sea coast between Tuapse and Psou (Starobogatov, 1962; Palatov, Vinarski, 2015; Vinarski, Kantor, 2016).

- Paladilhiopsis subovata Starobogatov, 1962. Stygobiont, known only from the type locality, a spring inside the Krasnoaleksandrovskaya (=Peshchera Ved'm) Cave, Lazarevsky City District, Greater Sochi, Krasnodar Province (Starobogatov, 1962; Vinarski, Kantor, 2016).

Order Geophila Ferrusac, 1821

Family Orculidae Steenberg, 1925

- Lauria sp. Likely troglobiont, recorded from the Shirokopokosskaya and Malaya Kazachebrodskaya caves, Greater Sochi, Krasnodar Province (Birstein, 1950; Ljovuschkin, 1966).

Family Trigonochlamydidae Hesse, 1882

- Troglolestes sokolovi Ljovuschkin and Matiokin, 1965. Troglobiont, known only from the type locality, the Vorontsovskaya Cave, Khostinsky City District, Greater Sochi, Krasnodar Province (Ljovuschkin, Matiokin, 1965).

Family Orculidae Steenberg, 1925

- Euxinolauria vitrea (Schileyko, 1988). Likely troglobiont, described from the Bolshaya Kazachebrodskaya (= VerkhneMzyimtinskaya) Cave near Mzymta River (Schileyko 1988), also recorded from the nearby Malaya Kazachebrodskaya (=Nizhne-Mzymtinskaya, Akhshtyrskaya) Cave, Adlersky City District, Greater Sochi, Krasnodar Province (Egorov, Greke, 2005).

Family Clausiliidae Gray, 1855

- Scrobifera taurica (L. Pfeiffer, 1848). Troglophile, recorded from the Shirokopokosskaya Cave, Khostinsky City District, Greater Sochi, Krasnodar Province, previously referred to as Clausilia foveicollis Parr. (Birstein, 1950).

- Serrulina sp. Troglophile, recorded from the Beloskalskaya Cave, as well as a cave on the Agura River, Greater Sochi, Krasnodar Province (Ljovuschkin, 1966). 
Family Zonitidae Morch, 1864

- Conulopolita cavatica (Riedel, 1966).

Troglobiont, described from the Shirokopokosskaya Cave (Khostinsky City District), also known from the nearby Bolshaya Kazachebrodskaya and Malaya Kazachebrodskaya caves (Adlersky City District), as well as the Chortova Nora Cave (Khostinsky City District), all in Greater Sochi, Krasnodar Province (Birstein, 1950; Ljovuschkin, 1966; Riedel, 1966; Koval, 2004a).

- Oxychilus (Longiphallus) sp. Likely troglophile, recorded from the Shakal'ya, Chortova Nora, Bolshaya Kazachebrodskaya, Malaya Kazachebrodskaya, Beloskalskaya, Shirokopokosskaya (=Bozhyey Materi) and Vorontsovskaya caves, all in Greater Sochi, Krasnodar Province (Birstein, 1950; Ljovuschkin, 1966; Koval, 2004a).

Class Bivalvia Linnaeus, 1758

Order Veneroida Adams et Adams, 1856

Family Euglesidae Pirogov et Starobogatov, 1974

- Euglesa (s. str.) personata (Malm, 1855). Likely stygophile, widespread in Europe, the Caucasus and Asia Minor (Kantor et al., 2010; Vinarski, Kantor, 2016), recorded from wells and springs near Adler, Greater Sochi, Krasnodar Province (Starobogatov, 1962). - Euglesa (s. str.) cavatica (Shadin, 1952). Stygobiont, described from a spring inside the Pionerskaya (= Ushchel'naya) Cave in the upper reaches of Vostochnaya (= Bolshaya) Khosta River, Khostinsky City District, Greater Sochi, Krasnodar Province. Reported also from caves in Abkhazia (Starobogatov, 1962; Chertoprud et al., 2016; Vinarski, Kantor, 2016, Turbanov et al., 2016a), but with some minor differences from the typical form.

Phylum Arthropoda Siebold, 1848

Subphylum Crustacea Brünnich, 1772

Class Copepoda Milne-Edwards, 1840

Order Cyclopoida Burmeister, 1834

Family Cyclopidae Rafinesque, 1815

- Acanthocyclops venustus stammeri (Kiefer, 1930). Stygobiont, interstitial-dweller.
Recorded from the imterstitial of Psezuapse River, Lazarevsky City District, Greater Sochi, and in the interstitial of Djubga River near Djubga, Tuapse District, both in the Krasnodar Province (Monchenko, 1984).

- Diacyclops bisetosus (Rehberg, 1880). Stygophile, recorded from the Bolshaya Kazachebrodskaya Cave, Adlersky City District, Greater Sochi, Krasnodar Province (Ljovuschkin, 1966).

- Paracyclops fimbriatus imminutus Kiefer, 1929. Stygophile, recorded from a karst spring on the Vostochnaya (= Bolshaya) Khosta River and from the Vorontsovskaya Cave, Khostinsky City District, Greater Sochi, Krasnodar Province (Borutzky, 1950a; Ljovuschkin, 1966).

- Speocyclops cinctus Monchenko, 1983. Interstitial of Alibek River, KarachaevoCherkessia (Monchenko, 1983).

- Speocyclops demetiensis (Scourfield, 1932). Stygobiont, interstitial-dweller. Recorded from the imterstitial of Psezuapse, Khosta, Mzymta and Psou rivers in the Krasnodar Province (Monchenko, 1986).

- Speocyclops lussianus Borutzky, 1950. Stygobiont, described from the Vorontsovskaya and Pionerskaya (= Ushchel'naya) caves, Khostinsky City District, Greater Sochi, Krasnodar Province (Borutzky, 1950a; Ljovuschkin, 1966).

- Speocyclops psezuapsensis Borutzky, 1965. Sygobiont, described from the Kirovskaya Cave, Psezuapse River, Lazarevsky City District, Greater Sochi, Krasnodar Province (Borutzky, 1965).

Order Harpacticoida G.O. Sars, 1903

Family Ameiridae Boeck, 1865

- Nitocrella hirta caucasica Borutzky, 1967. Stygobiont, described from the Zapovednaya (= Podparapetnaya)Cave, Khostinsky City District, Greater Sochi, Krasnodar Province (Borutzky, 1967a, 1972b).

- Megastygonitocrella ljovuschkini (Borutzky, 1967). Stygobiont, described or reported from the Dolgaya and Pionerskaya (= Ushchel'naya) caves near Sochi, and 
from the Krasnoaleksandrovskaya Cave near Lazarevskoe, all in the Krasnodar Province (Borutzky, 1967a, 1972b).

Family Canthocamptidae Brady, 1880

- Attheyella crassa (G.O. Sars, 1863). Stygophile, recorded from the Fanagoriyskaya Cave near Goryachiy Klyuch, from the Krasnoaleksandrovskaya Cave (Lazarevsky City District) and from the Pionerskaya (= Ushchel'naya), Labirintovaya and Dolgaya caves, Khostinsky City District, Greater Sochi, all in the Krasnodar Province (Borutzky, 1948a, 1972b).

- Bryocamptus aquaeductus Borutzky, 1934. Stygobiont, described from a water pipeline in the town of Teberda, Karachaevo-Chekessia (Borutzky, 1934).

- Bryocamptus innominatus Borutzky, 1940. Stygobiont, described from the Pionerskaya (= Ushchel'naya) Cave, Khostinsky City District, Greater Sochi, Krasnodar Province (Borutzky, Mikhailova-Neikova, 1970; Borutzky, 1972b).

- Bryocamptus pygmaeus (G.O. Sars, 1863). Stygophile, recorded from the Dolgaya and Labirintovaya caves, Khostinsky City District, Greater Sochi, Krasnodar Province (Borutzky, 1972b).

- Bryocamptus tarnogradskyi Borutzky, 1934. Stygophile, recorded from the Fanagoriyskaya Cave near Goryachiy Klyuch, from the Krasnoaleksandrovskaya Cave near Lazarevskoe, and from the Labirintovaya, Podparapetnaya (= Zapovednaya) and Tonnel Kuzmenko caves near Khosta, Greater Sochi, all in the Krasnodar Province (Borutzky, 1948a, 1972b; Ljovuschkin, 1966).

- Bryocamptus zschokkei caucasicus Borutzky, 1930. Stygophile, recorded from the Bolshaya Kazachebrodskaya (= Verkhne-Mzymtinskaya), Pionerskaya (=Ushchel'naya), Labirintovaya and Dolgaya caves near Sochi, from the Krasnoaleksandrovskaya Cave near Lazarevskoe, and from the Fanagoriyskaya Cave near Goryachiy Klyuch, all in the Krasnodar Province (Borutzky, 1948a, 1972b).
- Bryocamptus hostensis Borutzky, 1972. Likely stygobiont, described from the Labirintovaya Cave, Khostinsky City District, Greater Sochi, Krasnodar Province (Borutzky, 1972b).

- Bryocamptus sp. The first copepodid instar recorded from the Vorontsovskaya Cave, Khostinsky City District, Greater Sochi, Krasnodar Province (Borutzky, 1952).

- Canthocamptus staphylinus (Jurine, 1820). Stygophile, recorded from the karst waters on the Agura River, Greater Sochi, Krasnodar Province (Ljovuschkin, 1966).

- Pilocamptus georgevitchi (Chappuis, 1924). Stygobiont, recorded from the Krasnoaleksandrovskaya Cave (Lazarevsky City District), from the Dolgaya, Labirintovaya and Nikity caves, Greater Sochi, Krasnodar Province (Borutzky, Mikhailova-Neikova, 1970; Borutzky 1972b).

- Elaphoidella bidens coronata (G.O. Sars, 1904). Stygophile, discovered in the interstitial (Chappuis pit) of Mzymta River at Adler, Greater Sochi, Krasnodar Province (Borutzky, 1972b).

- Elaphoidella czerkessica Borutzky, 1972. Stygobiont, described from the Pionerskaya (= Ushchel'naya) Cave, Khostinsky City District, Greater Sochi, Krasnodar Province (Borutzky, 1972b).

- Moraria operculata Borutzky, 1948. Stygobiont, described from the Bolshaya Kazachebrodskaya (= Verkhne-Mzymtinskaya) and Dolgaya caves, as well as from the interstitial (Chappuis pit) at a bank of the Mzymta River, all in Greater Sochi, Krasnodar Province (Borutzky, 1948a, 1972b).

- Moraria hostensis Borutzky, 1972. Stygobiont, described from the Zapovednaya (= Podparapetnaya) Cave, Khostinsky City District, Greater Sochi, Krasnodar Province (Borutzky, 1972b).

- Moraria poppei (Mrazek, 1893). Stygobiont, recorded from the Dolgaya Cave, Khostinsky City District, Greater Sochi, Krasnodar Province (Borutzky, 1972b). - Moraria varica (Graeter, 1910). Stygobiont, recorded from the Fanagoriyskaya 
Cave near Goryachiy Klyuch and from the Labitintovaya Cave, Khostinsky City District, Greater Sochi, both in the Krasnodar Province (Borutzky, 1972b).

- Moraria sp. Nauplia recorded from the Dolgaya Cave, Khostinsky City District, Greater Sochi, Krasnodar Province (Borutzky, 1972b).

Family Parastenocarididae Chappuis, 1940

- Parastenocaris tenuis Borutzky, 1948. Stygobiont, described from the Fanagoriyskaya Cave near Goryachiy Klyuch, Krasnodar Province (Borutzky, 1948a, 1972b).

- Parastenocaris sp. Stygobiont, recorded from the interstitial waters on the banks of Mzymta River (Ljovuschkin, 1966).

Class Ostracoda Latreille, 1802

Order Podocopida G.O. Sars, 1866

Family Candonidae Kaufmann, 1900

- Trapezicandona ljovuschkini (Rudjakov, 1963). Stygobiont, described and still known only from the Krasnoaleksandrovskaya (= Peshchera Ved'm) Cave, Lazarevsky City District, Greater Sochi, Krasnodar Province (Rudjakov, 1963).

- Candona sp. Recorded without an exact species identification from the Krasnoaleksandrovskaya (= Peshchera Ved'm) Cave (Lazarevsky City District) and the Labirintovaya Cave (Khostinsky City District), both in Greater Sochi, Krasnodar Province (Rudjakov, 1963).

Family Cyprididae Baird, 1845

- Cypria reptans Bronstein, 1928. Crenobiont, found in the Dolgaya Cave, Khostinsky City District, Greater Sochi, Krasnodar Province (Rudjakov, 1963).

Family Limnocytheridae Klie, 1938

- Kovalevskiella (Cordocythere) rudjakovi (Danielopol, 1969). Stygobiont, described and still known only from the Krasnoaleksandrovskaya (= Peshchera Ved'm) Cave, Lazarevsky City District, Greater Sochi, Krasnodar Province (Danielopol, 1969). Earlier referrred to as Metacypris sp. (Rudjakov, 1963).

Class Malacostraca Latreille, 1817

Superorder Syncarida Packard, 1885
Order Bathynellacea Chappuis, 1915

Family Bathynellidae Grobbon, 1904

- Antrobathynella stammeri ciscaucasica (Birstein et Ljovuschkin, 1964). Stygobiont, interstitial-dweller, described from a spring in the Fanagoriyskaya Cave near Goryachiy Klyuch, Krasnodar Province (Birstein, Ljovuschkin, 1964, 1967b; Serban, 1993).

- Bathynella natans natans Vejdovsky, 1882. Stygobiont, interstitial-dweller, discovered in a waterbody inside the Pionerskaya (= Ushchel'naya) Cave, upper reaches of Vostochnaya (= Bolshaya) Khosta River, Greater Sochi, Krasnodar Province (Birstein, Ljovuschkin, 1967b).

Order Isopoda Latreille, 1817

Suborder Asellota Latreille, 1803

Family Asellidae Latreille 1803, sensu Rafinesque-Schmaltz, 1815

- Proasellus linearis (Birstein, 1967). Stygobiont, described from a spring at Evstafieva Shchel' near Gelendjik, Krasnodar Province (Birstein, 1967). There are also records of $P$. cf. linearis from near Tuapse, in springs and oozing waters in the valleys of Agoy and Nebug rivers, as well as in streams of Kadosh Park where the animals are washed out from subterranean waters during strong rain flashes (Sokolova, Palatov, 2015).

- Proasellus ljovuschkini (Birstein, 1967). Stygobiont, described from a karst vaucluse in the lower reaches of Khosta River, Greater Sochi, Krasnodar Province (Birstein, 1967).

- Proasellus similis (Birstein, 1967). Stygobiont, described from a waterbody inside the Nikity Cave in the Psakho River basin, a tributary of Kudepsta River, Greater Sochi, Krasnodar Province (Birstein, 1967). - Proasellus sp. (Fig. 5A). Stygobiont, possibly a new species, recorded from the Avgust Cave, Adlersky City District, Greater Sochi, Krasnodar Province (Turbanov et al., 2016a).

Family Microparasellidae Karaman, 1933 - Microcharon tantalus Birstein et Ljovuschkin, 1965. Stygobiont, described from 


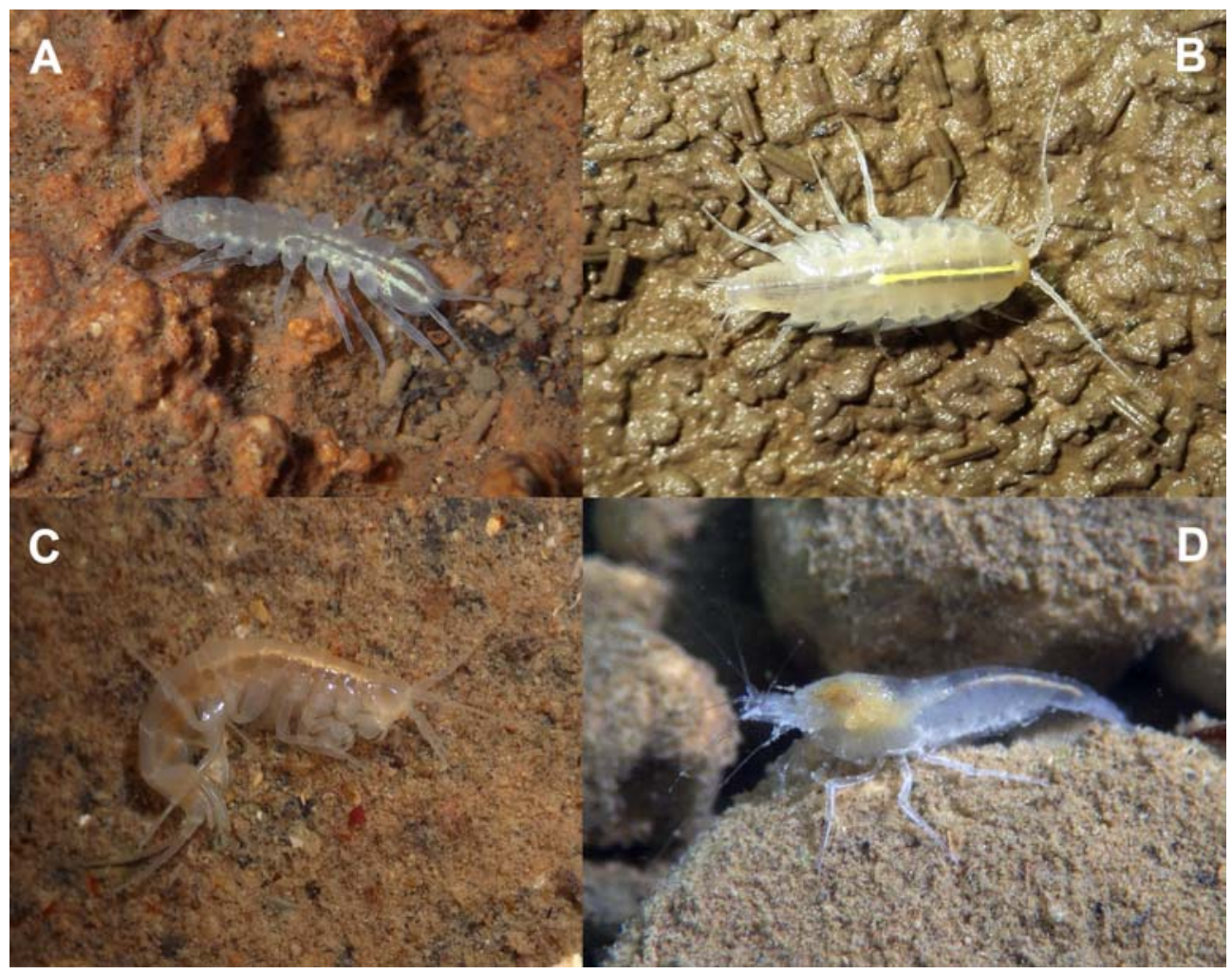

Fig. 5. Some stygo- or troglobiont crustaceans in nature (all caves from Greater Sochi, Krasnodar Province). A - Proasellus sp., a stygobiont water louse from the Avgust Cave; B - Ligidium cavaticum Borutzky, 1950, a troglobiont wood louse from the Giganov Cave; C - Niphargus sp., a stygobiont amphipod from the Gigantov Cave; D - Troglocaris (Xyphocaridinella) jusbaschjani Birstein, 1948, a stygobiont shrimp from sulphurous springs on the Agura River. All pictures by Ilya Turbanov.

Рис. 5. Некоторые стиго- и троглобионтные ракообразные в природе (все пещеры из Большого Сочи, Краснодарский край). А - Proasellus sp., стигобионтный водяной ослик из пещеры Август; В Ligidium cavaticum Borutzky, 1950, троглобионтная мокрица из пещеры Гигантов; C - Niphargus sp., стигобионтный бокоплав из пещеры Гигантов; D — Troglocaris (Xyphocaridinella) jusbaschjani Birstein, 1948, стигобионтная креветка из серных ручьев у реки Агура. Все фотографии Ильи Турбанова.

the interstitial of Mzymta River, Greater Sochi, Krasnodar Province (Birstein, Ljovuschkin, 1965).

Suborder Oniscidea Latreille, 1802

Family Ligiidae Brandt, 1883

- Ligidium zaitzevi Borutzky, 1950. Troglophile, described from a series of syntypes stemming from the Vorontsovskaya Cave, Khostinsky City District, Greater Sochi, Krasnodar Province, as well as the Mikhailovskaya Cave and the cave near Venetian Bridge, both latter near Sukhum, Abkhazia (Borutzky, 1950b, 1972a).
- Ligidium hypnorum (Cuvier, 1792). Troglophile, recorded for the Shirokopokosskaya Cave, Greater Sochi, Krasnodar Province (Borutzky, 1950b, 1972a).

- Ligidium cavaticum Borutzky, 1950 (Fig. 5B). Troglobiont, described from a series of syntypes coming from the Pervomayskaya, Podzemnaya Khosta, Shirokopokosskaya (= Bozhyey Materi), Vorontsovskaya, Beloskalskaya, Navalishinskaya, Partizanskaya, Pionerskaya (=Ushchel'naya), Zapovednaya (= Podparapetnaya), Malaya Kazachebrodskaya, Bolshaya Kazachebrod- 
skaya, Kamenskaya and Giganov (new data) caves, all in Greater Sochi, Krasnodar Province (Borutzky, 1950b, 1972a; Ljovuschkin, 1966).

Family Buddelundiellidae Verhoeff, 1930 - Pseudobuddelundiella hostensis Borutzky, 1967. Troglobiont, described from the Labirintovaya and Pionerskaya (=Ushchel'naya) caves, Greater Sochi, Krasnodar Province (Borutzky, 1967b, 1972a).

- Pseudobuddelundiella ljovuschkini Borutzky, 1967. Troglobiont, described from the Kirovskaya Cave, Lazarevsky City District, Psezuapse River, Krasnodar Province (Borutzky, 1967b, 1972a).

Family Trichoniscidae Sars, 1899

- Caucasocyphonethes cavaticus Borutzky, 1948. Troglobiont, represented by 4 subspecies from caves on the Mzymta, Khosta and Kudepsta rivers, Greater Sochi, Krasnodar Province: C. c. cavaticus Borutzky, 1948 from the Malaya Kazachebrodskaya Cave; C. c. chostensis Borutzky, 1948 from the Podzemnaya Khosta and Beloskalskaya caves; C. c. msymticus Borutzky, 1948 from the Bolshaya Kazachebrodskaya Cave; and C. c. adlerensis Borutzky, 1948 from the Shirokopokosskaya and Nikity caves (Borutzky, 1948b, 1972a).

- Psachonethes czerkessicus Borutzky, 1969. A troglomorphic species described from a cave on the Psakho River, a tributary of Kudepsta River, Greater Sochi, Krasnodar Province (Borutzky, 1969c, 1972a).

- Trichoniscus pygmaeus tuapsensis Borutzky, 1972. Troglobiont, described from a cave on the Psebe River, Tuapse District, Krasnodar Province (Borutzky, 1972a).

- Trichoniscus sp. A record is available of a troglomorphic woodlouse from the Shirokopokosskaya Cave, Khostinsky City District, Greater Sochi, Krasnodar Province (Borutzky, 1972a).

Family Cylisticidae Verhoeff, 1949

- Cylisticus birsteini Borutzky, 1961. Troglophile, described from a cave on the Psakho River, a tributary of Kudepsta River, recorded from the Navalishinskaya (= Mu- zeynaya) and Podzemnaya Khosta (= Khostinskaya Mokraya) caves, Greater Sochi, Krasnodar Province(Borutzky, 1961, 1972a; Ljovuschkin, 1966).

Order Amphipoda Latreille, 1816

Family Crangonyctidae Bousfield, 1973

- Lyurella shepsiensis Sidorov, 2015. Stygo- and crenobiont, described from springs in the Shepsi River basin (Sidorov 2015), also recorded in springs of the Ashe River basin near Kalezh and in the Krasnoaleksandrovskaya Cave (Lazarevsky City District), all in the Krasnodar Province (Sokolova, Palatov, 2015).

Family Niphargidae Bousfield, 1977

- Niphargus abchasicus Martynov, 1932. Stygophile, described from springs in the interfluve of Khosta and Kudepsta rivers, Greater Sochi, Krasnodar Province (İartynov, 1932), also recorded there later (Ljovuschkin 1963), as well as from springs within and near Sochi (Derzhavin, 1945). - Niphargus caelestis G.S. Karaman, 1982 (=N. stygius longidactylus Birstein, 1952). Stygobiont, described from the Pionerskaya (=Ushchel'naya) Cave, Greater Sochi, Krasnodar Province (Birstein, 1952). According to the Code of Zoological Nomenclature the original name was changed since "longidactylus" had been preoccupied by N. kochianus longidactylus Ruffo, 1937, from springs at Verona, Italy (Karaman, 1982).

- Niphargus cubanicus Birstein, 1954. Likely stygophile, described from a wellwarmed fish pond at Goryachiy Klyuch, Krasnodar Province (Birstein, 1954).

- Niphargus krasnodarus Karaman, 2012. Stygobiont, described from the Fanagoriyskaya Cave near Goryachiy Klyuch, Krasnodar Province (Karaman, 2012).

- Niphargus latimanus Birstein, 1952 (=N. stygius latimanus Birstein, 1952). Stygobiont, described from a stream in the Vorontsovskaya Cave, Greater Sochi, Krasnodar Province (Birstein, 1952). Morphologically similar forms recorded also from springs near Tuapse (Sokolova, Palatov, 2015). 
- Niphargus cf. magnus Birstein, 1940. Stygobiont, a possibly new species morphologically similar to N. magnus, found in springs near Tuapse, Krasnodar Province (Sokolova, Palatov, 2015).

- Niphargus potamophilus Birstein, 1954. Likely stygophile, described from experimental fish ponds in the mouth of Don River near the city of Rostov-on-Don, Rostov Region, as well as in Akhtyrskie fish ponds in the mouth of Kuban River, Abinsk District, Krasnodar Province (Birstein, 1954). - Niphargus pseudolatimanus Birstein, 1952 (= N. stygius pseudolatimanus Birstein, 1952). Stygobiont, described from a stream inside the Labirintovaya (= Novaya) Cave, Greater Sochi, Krasnodar Province (Birstein, 1952).

- Niphargus smirnovi Birstein, 1952. Stygophile, described from the Bolshaya Kazachebrodskaya (= Verkhne-Mzymtinskaya) Cave in the valley of Mzymta River, Greater Sochi, Krasnodar Province (Birstein, 1952). Subsequently recorded in springs of Khosta, Kudepsta and Psou rivers, as well as in the Vorontsovskaya, Labirintovaya, Dolgaya, Pionerskaya (= Ushchel'naya) and Podzemnaya Khosta caves in the same region. The species is thereby fairly variable, each river basin and cave system supporting its own unique morphotype. Perhaps we face a group of closely related species (Ljovuschkin, 1963). - Niphargus submersus (Derzhavin, 1945) (= Martynovia submersa Derzhavin, 1945). Stygophile, described from Sochinka River under a bridge in the town of Greater Sochi, Krasnodar Province (Derzhavin, 1945). Subsequently recorded in springs in the Vostochnaya (= Bolshaya) Khosta River basin (Ljovuschkin, 1963).

- Niphargus sp. (Fig. 5C). Stygobiont, possibly a new species, recorded from the Gigantov Cave in the Alek Massif, Greater Sochi, Krasnodar Province (new data).

Family Typhlogammaridae Bousfield, 1977 - Zenkevitchia sp. Stygobiont, a new, yet undescribed species recorded in the Avgust
Cave, Adlersky City District, Greater Sochi, Krasnodar Province (Turbanov, Marin, 2015, 2017).

Order Decapoda Latreille, 1802

Infraorder Caridea Dana, 1852

Family Atyidae De Haan, 1849

- Troglocaris (Xyphocaridinella) jusbaschjani Birstein, 1948 (Fig. 5D). Described and still known only from the type locality, i.e. hydrogen sulfide springs on the Agura River, Greater Sochi, Krasnodar Province (Birstein, 1948; Marin, Sokolova, 2014).

Infraorder Brachyura Linnaeus, 1758

Family Potamidae Ortmann, 1896

- Potamon ibericum tauricum (Czerniavsky, 1884). Possibly troglophile, recorded from the Chortova Nora Cave, Agura River, Greater Sochi, Krasnodar Province (Koval, 2004a).

Class Arachnida Cuvier, 1812

Order Aranei Clerck, 1758

There seem to be no troglobitic spiders not only in the Russian Caucasus, but across the entire former Soviet Union (Turbanov et al., 2016b).

Family Agelenidae C.L. Koch, 1837

- Tegenaria abchasica Charitonov, 1941. Troglophile, recorded from the Beloskal'skaya Cave, Khostinsky City District, Greater Sochi, Krasnodar Province (Turbanov, Nadolny, 2017).

- Tegenaria pontica Charitonov, 1947. Troglophile, described from the Fanagoriyskaya Cave near Goryachiy Klyuch, Krasnodar Province (Charitonov, 1947).

- Tegenaria sp. Troglophile, a record is available of a closer unidentified species from the Chortova Nora Cave, Khostinsky City District, Greater Sochi (Koval, 2004a).

Family Hahniidae Bertkau, 1878

- Iberina ljovuschkini Pichka, 1965. Troglophile, described from the Shakal'ya Cave, Lazarevsky City District, Greater Sochi, Krasnodar Province (Pichka, 1965).

Family Linyphiidae Blackwall, 1859

- Troglohyphantes birsteini Charitonov, 1947. Troglophile, described from the Bolshaya Kazachebrodskaya and Malaya Kazachebrodskaya caves, Greater Sochi, 
Krasnodar Province (Charitonov, 1947). Further records concern the Chortova Nora, Labirintovaya, Vorontsovskaya and Ametist caves, as well as a cave on Kamenka River, all in the same region (Pichka, 1965; Koval, 2004a; Turbanov, Nadolny, 2017). Family Nesticidae Simon, 1894

- Aituaria pontica (Spassky, 1932) (=Nesticus ponticus Spassky, 1932). Troglophile, described from a wine cellar at Khosta, Greater Sochi, Krasnodar Province (Spassky 1932). Later recorded from the Bolshaya Kazachebrodskaya, Chortova Nora and Ametist caves in the same region, as well as in a cave in the middle flow of Psakho River and a karst pit on Agura River, both also in the Krasnodar Province (Charitonov, 1947; Pichka, 1965; Koval, 2004a; Turbanov, Nadolny, 2017).

- Carpathonesticus birsteini (Charitonov, 1947). Troglophile, described from the Podzemnaya Khosta (in the publication of Charitonov it is indicated as Khostinskaya Mokraya) Cave, Greater Sochi, Krasnodar Province (Charitonov, 1947). Subsequently found in the Akhunskaya, Beloskal'skaya, Vorontsovskaya, Labirintovaya (=Novaya), Kamenskaya and Dolgaya caves, as well as in a cave on the Agura River in the same region (Pichka, 1965; Ljovuschkin, 1966; Koval, 2004a, b; Turbanov, Nadolny, 2017). - Carpathonesticus ljovuschkini (Pichka, 1965). Troglophile, described from the Shakal'ya Cave, Lazarevsky City District, Greater Sochi, Krasnodar Province (Pichka, 1965).

- Carpathonesticus zaitzevi (Charitonov, 1939). Troglophile, recorded from the Baribana Cave in the Alek Massif, Greater Sochi, Krasnodar Province (Turbanov, Nadolny, 2017).
- Carpathonesticus sp. Troglophile, recorded from the Beloskal'skaya Cave, Greater Sochi, Krasnodar Province (Turbanov, Nadolny, 2017).

Family Pholcidae C.L. Koch, 1851

- Hoplopholcus longipes (Spassky, 1934). Troglophile, described from a wine cellar at Khosta, Greater Sochi, Krasnodar Province (Spassky, 1934). Subsequently recorded in the Bolshaya Kazachebrodskaya (= Verkhne-Mzymtinskaya), Shirokopokosskaya, Beloskal'skaya and BS-2 caves, all in the same region (Charitonov 1941; Turbanov, Nadolny, 2017).

Family Tetragnathidae Menge, 1866 - Meta bourneti Simon, 1922. Troglophile, recorded from the Chortova Nora Cave, Khostinsky City District, Greater Sochi (Koval, 2004a), widespread in Europe.

- Metellina merianae (Scopoli, 1763). Troglophile, recorded from the Fanagoriyskaya and Shirokopokosskaya caves in the Krasnodar Province (Charitonov, 1947), widespread in Europe.

Order Pseudoscorpiones De Geer, 1778

Family Neobisiidae Chamberlin, 1930

- Neobisium (Neobisium) speleophilum Krumpal, 1986. Troglobiont, first recorded without an exact species identification from the Dolgaya and Pionerskaya caves in the Vorontsovka Massif near Adler, Greater Sochi, Krasnodar Province (Birstein, Ljovuschkin, 1960), later described (Krumpal, 1986).

- Neobisium sp. (Fig. 6A). Several records are available from caves in the Krasnodar Province: Shkol'naya and Gigantov (new data) in the Alek Massif, Pechal'naya in the Dzykhra Massif, and Dolgaya, Pionerskaya (=Ushchel'naya), Labirintovaya (Khostinsky City District), Greater Sochi (Ljovus-

пещеры Гигантов; В - Nemaspela sokolovi (Ljovuschkin et Starobogatov, 1963), троглобионтный сенокосец из пещеры Гигантов; C — Heterocaucaseuma feminaepectorum Antić et Makarov, 2016, троглобионтная двупарноногая многоножка из пещеры Гигантов; D — Dolichopoda euxina Semenov, 1901, троглофильный кузнечик из пещеры Ахунская; E - Caucasaphaenops molchanovi Belousov, 1999, троглобионтный жук-жужелица из пещеры Гигантов; F — Seracamaurops komarovi Hlaváč, Kodada et Koval, 1999, троглобионтный жук-ощупник из пещеры Гигантов. Все фотографии Ильи Турбанова. 


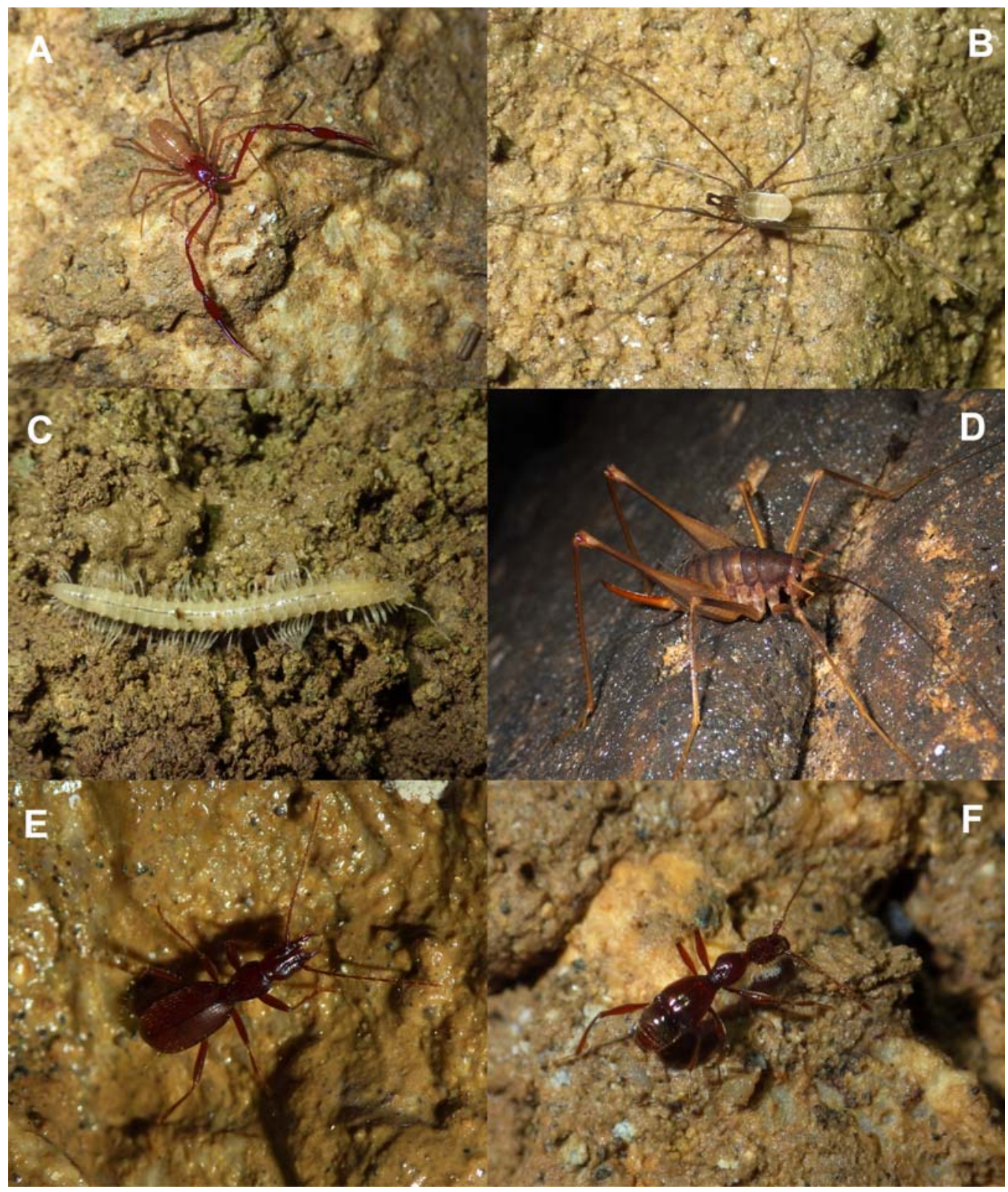

Fig. 6. Some troglobiont or troglophile arthropods in nature (all caves from Greater Sochi, Krasnodar Province). A - Neobisium sp., a troglobiont false scorpion from the Gigantov Cave; B - Nemaspela sokolovi (Ljovuschkin et Starobogatov, 1963), a troglobiont harvestman from the Gigantov Cave; C Heterocaucaseuma feminaepectorum Antić et Makarov, 2016, a troglobiont millipede from the Gigantov Cave; D - Dolichopoda euxina Semenov, 1901, a troglophile grasshopper from the Akhunskaya Cave; E Caucasaphaenops molchanovi Belousov, 1999, a troglobiont carabid beetle from the Gigantov Cave; F Seracamaurops komarovi Hlaváč, Kodada et Koval, 1999, a troglobiont pselaphid beetle from the Gigantov Cave. All pictures by Ilya Turbanov.

Рис. 6. Некоторые троглобионтные и троглофильные членистоногие в природе (все пещеры из Большого Сочи, Краснодарский край). А - Neobisium sp., троглобионтный ложноскорпион из 
chkin, 1966; Dashdamirov, Schawaller, 1992; Turbanov et al., 2016b).

Order Opiliones Sundevall, 1833

Family Nemastomatidae Simon, 1872

- Histricostoma caucasicum (Redikorzev, 1936). Likely troglophile, Caucasian endemic, recorded from the Fanagoriyskaya Cave near Goryachiy Klyuch, Krasnodar Province (Martens, 2006).

- Nemaspela abchasica (Ljovuschkin et Starobogatov, 1963). Troglobiont, recorded from the Beloskal'skaya, Kolokol'naya and Akhunskaya caves, Greater Sochi, Krasnodar Province (Martens, 2006; Chemeris, 2009).

- Nemaspela kovali Chemeris, 2009. Troglobiont, described from the Fontanka, Omega-12, Omega-15 and Otte-Shik caves in Kabardino-Balkaria (Chemeris, 2009).

- Nemaspela sokolovi (Ljovuschkin et Starobogatov, 1963) (Fig. 6B). Troglobiont, known from a few caves in the Greater Sochi region (Dolgaya, Labirintovaya, Vorontsovskaya and Gigantov (new data)), anophthalmic with elongated appendages (Ljovuschkin, Starobogatov, 1963; Martens, 2006; Chemeris, 2009).

- Nemaspela sp. Troglobiont, without an exact species identification recorded from the Akhunskaya Cave, Greater Sochi, Krasnodar Province (Koval, 2004b).

Family Phalangiidae Latreille, 1802

- Nelima pontica Charitonov, 1941. Likely troglophile, recorded from the Shirokopokosskaya, Bolshaya Kazachebrodskaya (= Verkhne-Mzymtinskaya) and Malaya Kazachebrodskaya (= Nizhnyaya Kazachebrodskaya, Nizhne-Mzymtinskaya) caves, Greater Sochi, Krasnodar Province (Charitonov, 1947; Ljovuschkin, Starobogatov, 1963; Ljovuschkin, 1966).

Subclass Acari Leach, 1817

Superorder Acariformes Zakhvatkin, 1952 Order Sarcoptiformes Reuter, 1909

Suborder Oribatida Van der Hammen, 1968

Family Phthiracaridae Perty, 1841

- Phthiracarus globosus (C.L. Koch, 1841).

Troglophile, recorded from the Voronts- ovskaya Cave, Khostinsky City District, Greater Sochi, Krasnodar Province (Ljovuschkin, 1966).

Superorder Parasitiformes Reuter, 1909

Order Mesostigmata Canestrini, 1891

Suborder Ixodoidea Leach, 1815

Family Ixodidae C.L. Koch, 1844

- Ixodes vespertilionis C.L. Koch, 1844. A parasite of bats, recorded from the Pionerskaya (=Ushchel'naya) and Dolgaya caves, Greater Sochi, Krasnodar Province (Ljovuschkin, 1966).

Subphylum Tracheata Haeckel, 1866

Class Chilopoda Latreille, 1817

Order Lithobiomorpha Pocock, 1895

Family Lithobiidae Newport, 1844

- Harpolithobius perplexus Zalesskaja, 1973. Troglophile, recorded from the Chortova Nora Cave, Greater Sochi, Krasnodar Province (Koval, 2004a).

- Lithobius liber Lignau, 1903. Troglophile, recorded from the Chortova Nora Cave, Greater Sochi, Krasnodar Province, but perhaps belongs to a different species (Volkova, 2015).

- Lithobius reconditus Zalesskaja, 1973. Troglophile, recorded from the Pionerskaya (=Ushchel'naya) Cave, Greater Sochi, Krasnodar Province (Zalesskaja, 1973a, b, 1978). - Lithobius stuxbergii Sseliwanoff, 1881. Troglophile, recorded from the Vorontsovskaya, Shirokopokosskaya and Chortova Nora caves, all in Greater Sochi, Krasnodar Province(Zalesskaja, 1963, 1973a, b, 1978; Koval, 2004a).

Class Diplopoda de Blainville in Gervais, 1844 Order Glomerida Leach, 1814

Family Glomeridellidae Cook, 1896

- Typhloglomeris caucasica Golovatch, 1975. Troglophile, recorded from several caves near Sochi, Krasnodar Province (Partizanskaya, Dolgaya, Vorontsovskaya etc.) (Golovatch, 1975), but then found epigeically at Khosta (Golovatch, Chumachenko, 2013).

Family Glomeridae Leach, 1815

- Trachysphaera costata (Waga, 1857).

Troglophile, widespread across Central, 
Eastern and Southern Europe, as well as Anatolia to northwestern Iran, recorded from a cave in Crimea, from the Fanagoriyskaya Cave near Goryachiy Klyuch, and from a karst funnel at Shedok, both Krasnodar Province (Golovatch, 1990).

- Trachysphaera minuta Golovatch, 1976. Troglophile, known from two caves near Sochi - Partizanskaya, Malaya Kazachebrodskaya (= Nizhne-Kazachebrodskaya), as well as epigeically across much of the Caucasus (Russia, Georgia and Azerbaijan) (Golovatch, 1990).

- Hyleoglomeris sp. Troglophile or trogloxene, from a tectonic cave in the upper basin of Psebe River, Tuapse District, Krasnodar Province (Golovatch, 1984-1985).

Order Chordeumatida Pocock, 1894

Family Anthroleucosomatidae Verhoeff, 1899

- Caucaseuma elephantum Antić et Makarov, 2016. Troglobiont, known only from the Yuzhnyi Slon Cave, Mt Dzentu, ca 1800 m a.s.1., Karachaevo-Cherkesskaya Republic, northern Caucasus; body yellowish white, 11-12 ocelli in 3-4 rows (Antić, Makarov, 2016).

- Caucaseuma fanagoriyskaya Antić et Makarov, 2016. Troglobiont, known only from the Fanagoriyskaya Cave near Goryachiy Klyuch, Krasnodar Province; body greyish yellow with a brown head, 21-24 ocelli in 6 rows (Antić, Makarov, 2016).

- Caucaseuma lohmanderi Strasser, 1970. Likely troglobiont, frequent in caves in the western Caucasus (near Sochi, Krasnodar Province): Baribana, Labirintovaya, Navalishinskaya (= Muzeynaya), Vorontsovskaya, and since the description, Ametist Cave (Western Akhtsu Mts), Akhunskaya, Partizanskaya, Dolgaya, Shirokopokosskaya (= Bozhyey Materi), Tisovaya (perhaps this is the Zapovednaya Cave) caves (Strasser, 1970; Antić, Makarov, 2016).

- Caucaseuma minellii Antić et Makarov, 2016. Likely troglobiont, known only from the Sukhaya and Bolshaya Azishskaya caves, both near Sochi, Krasnodar Prov- ince; body yellowish white, $22-24$ ocelli in 6 rows (Antić, Makarov, 2016).

- Heterocaucaseumafeminaepectorum Antić et Makarov, 2016 (Fig. 6C). Likely troglobiont, from the Krasnoyarskaya and Pechalnaya caves, both in the Dzykhra karst massif, and the Osennyaya, Medvezhya and Gigantov caves in the Alek Massif, Greater Sochi, Krasnodar Province; body yellowish white, 10-18 ocelli in 3-4 rows (Antić, Makarov, 2016; Antić et al., 2018).

- Metamastigophorophyllon giljarovi (Lang, 1959). Troglophile, widespread along the Black Sea coast from Tuapse to Abkhazia, recorded also from the Shirokopokosskaya $(=$ Bozhyey Materi, = OurLady) Cave, Greater Sochi, Krasnodar Province (Antić, Makarov, 2016).

Order Julida Brandt, 1833

Family Blanulidae C.L. Koch, 1847

- Nopoiulus (s. str.) ammonites Enghoff, 1984. Likely troglobiont, described from the Ammonitovaya Cave near Shedok, Psebai District, Krasnodar Province (Enghoff 1984), later recorded from the nearby Dedova Cave (Golovatch, Enghoff, 1990).

- Nopoiulus (s. str.) kochii (Gervais, 1847). Troglophile, abundant populations observed in the Bolshaya Kazachebrodskaya and Shirokopokosskaya caves near Sochi, Krasnodar Province (Golovatch, 1981, 1984/85; Golovatch, Enghoff, 1990).

Family Julidae Leach, 1814

- Archileucogeorgia sp. Troglophile?, recorded from the Fanagoriyskaya Cave near Goryachiy Klyuch, Krasnodar Province (Ljovuschkin, 1966).

- Cylindroiulus placidus (Lignau, 1903). Troglophile, widespread across the western Caucasus, including a record from the Meshok Cave, Maikopsky District, Adygea Republic (Lohmander, 1936).

- Cylindroiulus pterophylacum Read, 1992. Troglophile, widespread across the western Caucasus, including a record from the Beloskal'skaya Cave, Khostinsky City District, Greater Sochi, Krasnodar Province (Read, 1992). 
- Cylindroiulus schestoperovi Lohmander, 1932. Troglophile, widespread across the western Caucasus, including records from the Beloskal'skaya Cave, Khostinsky City District, Greater Sochi, Krasnodar Province, and a cave in western Georgia (Read, 1992).

Order Polydesmida Peach, 1815

Family Polydesmidae Peach, 1815

- Brachydesmus furcatus Lohmander, 1936.

Troglophile, widespread across the northwestern Caucasus, including records from the Fanagoriyskaya Cave near Goryachiy Klyuch, the Baribana and Akhunskaya caves (Golovatch et al., 2016), as well as the Medvezhya Cave (new data) near Sochi.

Family Paradoxosomatidae Daday, 1889

- Strongylosoma kordylamythrum Attems, 1898. Troglophile, widespread across the Caucasus, also recorded from the Psebe Cave near Tuapse, as well as the Shirokopokosskaya (= Bozhyey Materi) and Labirintovaya caves, Khostinsky City District, Greater Sochi, Krasnodar Province, and a cave in Abkhazia (Evsyukov et al., 2016). Family Trichopolydesmidae Verhoeff, 1910 - Caucasodesmus inexpectatus Golovatch, 1985. Troglobiont, unpigmented, antennae and legs elongated, from the Nyvdjinlagat (= Tagardonskaya) Cave in North Ossetia (Golovatch, 1984-1985).

Superclass Hexapoda Blainville, 1816

Class Collembola Lubbock, 1870

Order Poduromorpha Börner, 1913, sensu

D'Haese, 2002

Superfamily Hypogastruroidea Salmon, 1964, sensu Deharveng, 2004

Family Hypogastruridae Börner, 1906 - Typhlogastrura preobrazhenskyi Babenko, 1987. Troglobiont, described from the Shuby-Nykhasskaya Cave in North Ossetia (Babenko, 1987).

Order Entomobryomorpha Börner, 1916, sensu Soto-Adames et al., 2008

Superfamily Tomoceroidea Szeptycki, 1979 Family Tomoceridae Schäffer, 1896

- Plutomurus jeleznovodskii Kniss et Thibaud, 1999. Troglobiont with traits of tro- glomorphism, described from a cave near Zheleznovodsk, Stavropol' Province(Kniss, Thibaud, 1999; Kniss, 2006).

-Plutomurus kelasuricus Martynova, 1969.

Troglophile devoid of distinct troglomorphic features, recorded from the Baribana Cave, Alek Massif, Greater Sochi, Krasnodar Province (Kniss, Thibaud, 1999; Kniss, 2006).

- Plutomurus sorosii Kniss et Thibaud, 1999. Troglobiont with traits of troglomorphism, described from a cave in the Teberda Nature Reserve, Karachaevo-Cherkessia (Kniss, Thibaud, 1999; Kniss, 2006).

Class Insecta Linnaeus, 1758

Order Plecoptera Burmeister, 1839

Family Leuctridae Klapálek, 1905

- Leuctra furcatella Martynov, 1928. Trogloxene. Larvae and adults recorded from the grotto part of the Krasnoaleksandrovskaya Cave, Lazarevsky City District, Greater Sochi, Krasnodar Province (Palatov, Sokolova, 2015).

Family Nemouridae Newman, 1853

- Nemoura elegantula Martynov, 1928. Trogloxene. Larvae and adults recorded from the grotto part of the Krasnoaleksandrovskaya Cave, Lazarevsky City District, Greater Sochi, Krasnodar Province (Palatov, Sokolova, 2015).

Order Orthoptera Latreille, 1793

Family Rhaphidophoridae Walker, 1869 - Dolichopoda euxina Semenov, 1901 (Fig. 6D). Troglophile. Described from caves in the Caucasus (Semenov, 1901), being very common there. Known from caves in the Adygea Republic (Shapovalov et al., 2015), the Krasnodar Province, Abkhazia, western Georgia (Satunin, 1912; Birstein, Lopashov, 1940; Zaitsev, 1948; Birstein, 1950; Barjadze et al., 2015) and South Ossetia (Bey-Bienko, 1965, 1969), altogether over 40 records.

Order Trichoptera Kirby, 1813

Suborder Annulipalpia Martynov, 1924

Family Polycentropodidae Ulmer, 1903

- Plectrocnemia sp. sensu Lepneva, 1940.

Possible troglophile. In the Krasnodar Prov- 
ince, recorded from the Dolgaya Cave, Greater Sochi. Larvae live under stones and in weak currents (Lepneva, 1940; Palatov, Sokolova, 2015).

Order Coleoptera Linnaeus, 1758

Family Carabidae Latreille, 1802

- Caucasaphaenops molchanovi Belousov, 1999 (Fig. 6E) Troglobiont from the Sokolova and Gigantov (new data) caves, Greater Sochi, Krasnodar Province (Belousov, 1999).

- Caucasorites kovali Belousov, 1999. Troglobiont from the Akhunskaya Cave, Greater Sochi, Krasnodar Province (Belousov, 1999).

- Caucasorites kovali amplicolis Belousov, 1999. Troglobiont from the Psakho Cave, Greater Sochi, Krasnodar Province (Belousov, 1999).

- Caucasorites shchurovi Belousov et Zamotajlov, 1997. Troglobiont from the Entomologicheskaya Cave, Lazarevsky City District, Greater Sochi, Krasnodar Province (Belousov, Zamotajlov, 1997).

- Caucasorites victori Belousov, 1999. Troglobiont from the Akhunskaya Cave, Khostinsky City District, Greater Sochi, Krasnodar Province (Belousov, 1999).

- Cimmerites circassicus Reitter, 1888. A troglomorphic species not yet encountered in caves. Described from mountain regions of the Krasnodar Province, between Tuapse and Sochi. Characterized by reduced eyes and an unpigmented tegument (Reitter, 1888-1889; Winkler, 1926), likely adapted to the MSS.

- Cimmerites kryzhanovskii Belousov, 1999. Possible troglobiont, described from the Malaya Kazachebroskaya Cave, Adlersky City District, Greater Sochi, Krasnodar Province (Belousov, 1999).

- Cimmerites maximovitchi Belousov and Koval, 2011. Possible troglobiont, described from the Akhunskaya and Labirintovaya caves, Khostinsky City District, Greater Sochi, Krasnodar Province; occurring together with Caucasorites victori on the western slope of Mt Akhun, Sochi region (Belousov, Koval, 2011).

- Cimmerites zamotajlovi Belousov, 1998. Troglophile, described from forest litter on Mt Verblyudka near the village of Kalinovoe Ozero, Greater Sochi, Krasnodar Province. Recorded also from other epigean habitats near Khosta, as well as from the Vorontsovskaya Cave, all within Greater Sochi, Krasnodar Province (Belousov, 1998).

- Duvalius gusevi Belousov, 1989. A troglomorphic species not yet recorded from caves. Described from the bank of a mountain spring near the sources of Kezadon River, Irafskiy District, North Ossetia, where it occurs under large boulders (Belousov, 1989). Characterized by a light coloration and strongly reduced eyes, likely adapted to the MSS.

- Duvalius miroshnikovi Belousov et Zamotajlov, 1995. Troglobiont from the Baribana Cave, Greater Sochi, Krasnodar Province (Belousov, Zamotajlov, 1995).

- Jeannelius birsteini Ljovuschkin, 1965. Troglobiont from caves in Greater Sochi, Krasnodar Province (Vorontsovskaya, Akhunskaya, Beloskal'skaya) (Ljovuschkin, 1965).

- Jeannelius zhicharevi Lutshnik, 1915. A troglomorphic species not yet recorded from caves. Described from the Mt Achishkho karst massif near Sochi (Luchnik, 1915; Kryzhanovskij et al., 1995); a species related to J. gloriosus and J. magnificus, both from Abkhazia.

- Meganophthalmus irinae Belousov et Zamotajlov, 1999. Troglophile, described from the Chernogorye karst massif near Maikop, Adygea Republic, occurring both in epigean habitats under stones and in caves (Belousov, Zamotajlov, 1999).

- Meganophthalmus kravetzi Komarov, 1993. Troglobiont, described from the Fontanka Cave in the upper reaches of Belaya Rechka River, 15 km SW of Nalchik, Cherkesskiy District, Kabardino-Balkaria. Found $25 \mathrm{~m}$ off the cave entrance, eyes absent (Komarov, 1993; Belousov, Koval, 2009). 
- Nannotrechus ciscaucasiens (Ljovuschkin, 1972) (= Birsteiniotrechus ciscaucasiens Ljovuschkin, 1972). Likely troglobiont, found in the Ammonitovaya Cave near Psebai, Kuban River basin, Mostovskoy District, Krasnodar Province (Ljovuschkin, 1972a). This genus belongs to the Neotrechus lineage and is closely related to Troglocimmerites.

- Nannotrechus fishtensis Belousov, 1989. A troglomorphic species not yet recorded from caves. Described from near Mt Fisht, at the sources of Armyanka River, Mostovskoy District, Krasnodar Province. Characterized by reduced eyes and an unpigmented tegument (Belousov, 1989), likely adapted to the MSS.

- Nannotrechus kovali Belousov, 1989. A troglomorphic species not yet encountered in caves. Described from the montane areas near Solokh-Aul, Greater Sochi, Krasnodar Province (Belousov, 1989). Characterized by reduced eyes, likely adapted to the MSS.

- Porocimmerites imitator Belousov, 1998. A troglomorphic species not yet encountered in caves. Characterized by reduced eyes and a reddish coloration, occurring in the low flow region of Mzymta River near Sochi (Belousov, 1998), likely adapted to the MSS.

- Trechus heniochicus Ljovuschkin, 1970. Troglophile, described from the Vorontsovskaya Cave, Khostinsky City District, Greater Sochi, Krasnodar Province. Found on rotting wood (Ljovuschkin, 1970).

-Laemostenus koenigi (Reitter, 1887). Troglophile, recorded from the Fanagoriyskaya and Stalaktitovaya caves, both near Goryachiy Klyuch, Krasnodar Province (Vereshchagina, 1985; Vereshchagina, Makarov, 1986).

- Laemostenus tschitscherini Semenov, 1909. Troglophile, described from the Dakhovskaya Cave, Maikopsky District, Adygea Republic (Semenov-Tian-Shanskiy, 1909), also known from many caves near Maikop (Belaya, Sukhaya, Budkova etc.)
(Vereshchagina, 1985; Vereshchagina, Makarov, 1986).

- Pterostichus lacunosus (Chaudoir, 1844). Likely troglophile, recorded from the Vorontsovskaya Cave, Khostinsky City District, Greater Sochi, Krasnodar Province (Ljovuschkin, 1962).

Family Cryptophagidae Kirby, 1937

- Cryptophagus pilosus Gyllenhal, 1827. Troglophile, recorded from the Chortova Nora Cave, Khostinsky City District, Greater Sochi, Krasnodar Province (Koval, 2004a).

Family Curculionidae Latreille, 1802

- Otiorhynchus vargovitchi Davidian, 2007. Troglobiont, described from the Beloskal'skaya Cave, Khostinsky City District, Greater Sochi, Krasnodar Province (Davidian, 2007).

Family Elmidae Curtis, 1830

- Limnius colchicus Delève, 1963. Likely troglophile, both larvae and adults encountered in the waters of the Dolgaya Cave near Khosta, Greater Sochi, Krasnodar Province. Penetrating inside the cave up to $1 \mathrm{~km}$, but retaining the typical coloration (Palatov, Sokolova, 2015).

Family Leiodidae Fleming, 1821

- Catops subfuscus Kellner, 1846. Troglophile, recorded in a cave at Svetlaya Balka, Mostovskoy District, Adygea Republic, at Eki-Suara, Malokarachaevskiy District, Karachaevo-Cherkessia, and in a cave at Ispravnaya, Zelenchuk District, Karachaevo-Cherkessia (Perkovsky, 1991).

-Choleva sp. Subtroglophile, recorded from the Labirintovaya Cave, Khostinsky City District, Greater Sochi, Krasnodar Province (Ljovuschkin, 1962).

Family Pselaphidae Latreille, 1802 - Bryaxis balneator Besuchet et Kurbatov, 2007. Troglophile, recorded from the Akhunskaya Cave, Khostinsky City District, Greater Sochi, Krasnodar Province (Besuchet, Kurbatov, 2007), but also inhabiting epigean biotopes.

- Bryaxis kovali Besuchet et Kurbatov, 2007. Troglophile, recorded from the La- 
birintovaya Cave, Khostinsky City District, Greater Sochi, Krasnodar Province; showing no visible adaptations to subterranean life (Besuchet, Kurbatov, 2007).

- Seracamaurops komarovi Hlaváč, Kodada et Koval, 1999 (Fig. 6F). Troglobiont, described from the Baribana and Gigantov (new data) caves in the Alek Massif, Greater Sochi, Krasnodar Province (Hlaváč et al., 1999).

Family Ptiliidae Heer, 1843

- Ptenidium intermedium Wankowicz, 1896. Troglophile associated in its development with bat guano. Recorded from the Chortova Nora Cave, Khostinsky City District, Greater Sochi, Krasnodar Province (Koval, 2004a).

Family Scirtidae Fleming, 1821

- Odeles sp. Troglophile, larvae found under stones in a stream in places with a weakened current in the Dolgaya Cave, Khostinsky City District, Greater Sochi, Krasnodar Province (Palatov, Sokolova, 2015).

Family Staphylinidae Lameere, 1900

- Bisnius parcus (Sharp, 1874). Troglophile, recorded from the Chortova Nora Cave, Khostinsky City District, Greater Sochi, Krasnodar Province (Koval, 2004a). - Heinzia caucasica Gusarov et Koval, 2002. Troglophile, described the Baribana Cave in the Alek Massif, Greater Sochi, Krasnodar Province, recorded also from the Sokolova (= Atsinskaya), Dolgaya and Vorontsovskaya caves in the same area; showing no visible adaptations to subterranean life, encountered in the Caucasus in epigean habitats as well (Gusarov, Koval, 2002).

Order Diptera Linnaeus, 1758

Suborder Nematocera Schiner, 1862

Family Limoniidae Speiser, 1909

- Limonia nubeculosa Meigen, 1804. Troglophile, repeatedly recorded from Transcaucasian caves: Bolshaya Kazachebrodskaya, Malaya Kazachebrodskaya, Akhunskaya, Shirokopokosskaya, Vorontsovskaya etc., all within Greater Sochi, Krasnodar
Province, etc. (Birstein, Lopashov, 1940; Zaitsev, 1948; Birstein, 1950; Koval, 2004b).

Family Sciaridae Billberg, 1820

- Neosciara sp. Troglophile, recorded from the Bolshaya Kazachebrodskaya Cave, Adlersky City District, Greater Sochi, Krasnodar Province (Ljovuschkin, 1966).

Suborder Brachycera Zetterstedt, 1842

Family Heleomyzidae Bezzi, 1911

- Heteromyza atricornis Meigen, 1830.

Troglophile, recorded from the Bolshaya Kazachebrodskaya (= Verkhne-Mzymtinskaya), Akhunskaya and Chortova Nora caves, all within Greater Sochi, Krasnodar Province (Birstein, 1950; Koval, 2004a, b). Larvae dwelling in bat guano (Zaitsev, 1948).

Phylum Chordata Haeckel, 1874

Subphylum Vertebrata J.-B. Lamarck, 1801

Class Mammalia Linnaeus, 1758

Order Chiroptera Blumenbach, 1779

Caves play a vital role in the life-cycle of eight (out of 31 reported from the Russian Caucasus) bat species, which use them as hibernation, breeding and swarming sites. Another 16 species occur in caves and artificial undergrounds in some periods of the life cycle, but are not strictly dependent on them.

Family Rhinolophidae Gray, 1825

- Rhinolophus euryale Blasius, 1853. Seven distributional records of $R$. euryale were reported from the vicinity of Sochi, Krasnodar Province, Russia, including one nursery roost in a small cave near the Nizhnyaya Shilovka village (Gazaryan, 2016).

- Rhinolophus ferrumequinum (Schreber, 1774) (Fig. 7B). This typical cave-dwelling species is abundant in the western Caucasus, whereas it is rather rare in the central part of the Caucasus due to the deficit of suitable underground chambers. Nursery roosts and hibernating groups have been found in the Caucasus only in underground chambers of different origin, mainly in karst caves. At least 65 among nearly 100 summer and winter records were made in the latter roost type. 


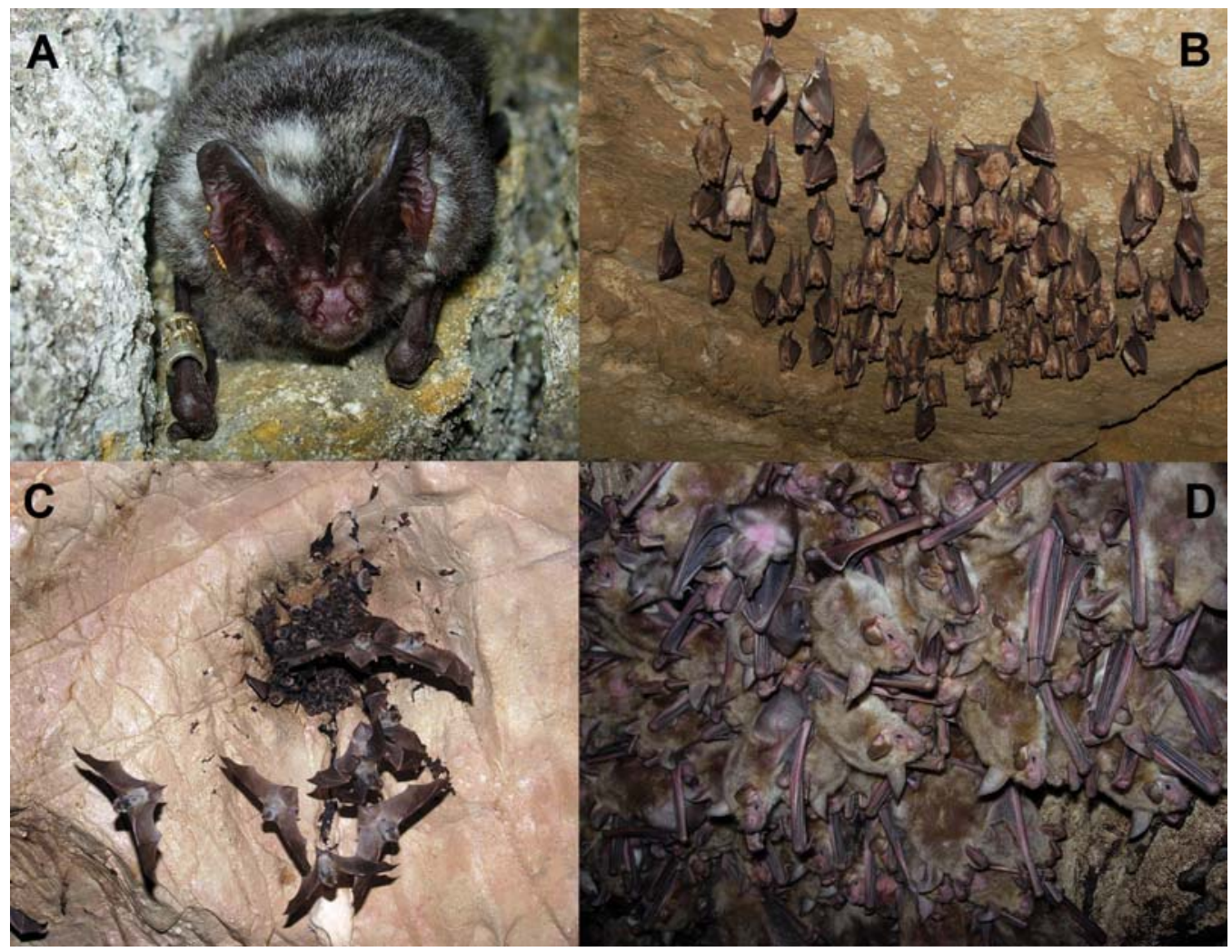

Fig. 7. Some bats from the Russian Great Caucasus in nature. A - a Ringed European barbastelle, Barbastella barbastellus (Schreber, 1774), hibernating on the wall of a mine near Derbentskaya, Seversky District, Krasnodar Province; B - a winter aggregation of Greater Horseshoe bats, Rhinolophus ferrumequinum (Schreber, 1774), in a gypsum cave near Psebai, Mostovskoy District, Krasnodar Province; C - a colony of Bent-winged bats, Miniopterus schreibersii (Kuhl, 1817), in a limestone cave near Guamka, Apsheronsky District, Krasnodar Province; D - a maternity roost of Lesser mouse-eared bats, Myotis blythii (Tomes, 1857), in a cave near Karabudakhkent, Dagestan. All pictures by Suren Gazaryan.

Рис. 7. Некоторые летучие мыши с Большого Кавказа России в природе. А - широкоушка европейская (Barbastella barbastellus (Schreber, 1774)), зимующая на стене шахты близ станицы Дербентская (Северский район Краснодарского края); В — зимнее скопление большого подковоноса (Rhinolophus ferrumequinum (Schreber, 1774)) в гипсовой пещере близ поселка Псебай (Мостовской район Краснодарского края); С — колония обыкновенного длиннокрыла (Miniopterus schreibersii (Kuhl, 1817)) в известковой пещере близ поселка Гуамка (Апшеронский район Краснодарского края); D - материнский насест остроухой ночницы (Myotis blythii (Tomes, 1857)) в пещере близ села Карабудахкент (Дагестан). Все фотографии Сурена Газаряна.

- Rhinolophus hipposideros (Borkhausen, 1797). The Lesser horseshoe bat is widely distributed across the region from the Utrish Peninsula in the West to the vicinity of Derbent in the Southeast. It hibernates in caves and other underground spaces, whilst maternity colonies overwhelmingly occupy above-ground roosts. In total, this bat has been reported from nearly 120 geographi- cal locations in the Russian Caucasus, nearly half of which are caves.

- Rhinolophus mehelyi Matschie, 1901. The only maternity and hibernation roost in Russia has been known since 1972 in the Karabudakhkentskaya Cave in Dagestan, with the number of bats gradually depleting from 1000 in 1972 to $30-40$ in 2008. 
Family Vespertilionidae Gray, 1821

- Barbastella barbastellus (Schreber, 1774)

(Fig. 7A). Barbastelles utilize caves and other underground shelters for hibernation and swarming. Of the ca 60 location records of the species, as many as 35 belong to caves. The hibernation colony in the Kanyon Cave, Krasnodar Province is the largest ever reported for the species, accumulating up to 8 thousand individuals in harsher winters. Another large winter aggregation of over 2,000 barbastelles was discovered in the Mayskaya Cave in KarachaevoCherkessia (Gazaryan, 1999, 2007a; Gazaryan, Panyutina, 2013).

- Barbastella caspica (Satunin, 1908). Amirkhanov (1980) reportedly found 6 individuals in two small caves near the Amushi and Tagada villages in Dagestan. The species was also recorded from caves in other parts of its distribution range.

-Eptesicus serotinus (Schreber, 1774). This species only occasionally hibernates in caves; several animals were found in the Kanyon Cave in winter, few serotines were also netted at the entrance to Babaylovskaya Cave in the Caucasian State Nature Reserve during swarming in August. In summer, resident males often occupy crevices and niches at the entrance parts of large caves and grottos.

- Hypsugo savii (Bonaparte, 1837). The species uses spacious caves and grottos as swarming sites. It was netted at the entrances to the neighbouring two caves in the Mzymta River valley near the city of Sochi: Bolshaya Kazachebrodskaya (Smirnov, 2001) and Dzykhrinskaya (= Lianovaya). - Myotis alcathoe von Helversen et Heller, 2001. Several individuals were netted entering or flying out from the Fanagoriyskaya and Krasnoaleksandrovskaya (= Takhira) caves, Krasnodar Province during swarming, one male was found hibernating in the Rozhnova Cave in Karachaevo-Cherkessia. - Myotis bechsteinii (Kuhl, 1817). Single hibernating individuals were found in the karst caves Fanagoriyskaya and Kanyon.
Swarming Bechstein's bats were netted at the entrances to the caves Malaya and Bolshaya Kazachebrodskaya (Tsytsulina, 1998; Smirnov, 2001), and Dzykhrinskaya (= Lianovaya), Akhunskaya and Fanagoriyskaya, all within the Krasnodar Province. - Myotis blythii (Tomes, 1857) (Fig. 7D). With ca 110 record locations, the Lesser Mouse-eared Bat occurs literally everywhere in montane ecosystems of the region. As everywhere within their range, these bats occupy both underground and aboveground roosts in summer, although hibernate only in caves and other underground sites. Large maternity colonies comprising hundreds of females are sustainably present in the caves Karabudakhkenskaya (Dagestan) and Samorodnaya (KarachaevoCherkessia); a mass winter aggregation amounting to over 2,000 bats is known from the Shubi-Nykhass Cave in North Ossetia (Gazaryan, 2007b).

- Myotis brandtii (Eversmann, 1845). Brandt's bat is common in woodland areas across the region and the absence of any records from Dagestan is apparently related to insufficient research efforts. Among 26 record locations in the Russian Caucasus, seven belong to the following caves: Fanagoriyskaya, Kanyon, Babaylovskaya and Tru52 in the Krasnodar Region, Mayskaya and Pogrebok in Karachaevo-Cherkessia, and Anglo-Russkaya in the Adygea Republic. During the swarming time, $M$. brandtii was abundant among bats netted at the entrances to these caves, even though only single solitarily hibernating Brandt's bats were found there in the winter.

- Myotis dasycneme (Boie, 1825). One young female was found on 8 November 2003 in the Gunkina-4 Cave in Karachaevo-Cherkessia (Gazaryan, 2004), but the occurrence has since been revealed nowhere else in the region. Consequently, we tend to associate this single record with a vagrant bat.

- Myotis daubentonii (Kuhl, 1817). Until the end of the 20th century, the species had 
been considered as extremely rare or even a recent colonist in the Caucasus and Transcaucasia. However, after extensive fieldwork the former putative status was reassessed to common and abundant across the region (Gazaryan et al., 2008). Although it was recorded at 82 locations in the Russian Caucasus, only several hibernation sites containing modest (up to 10 animals) numbers of Daubenton's bats have been found so far. Daubenton's bats do not aggregate in large clusters in the Caucasus and only sole animals could be observed in the caves Fanagoriyskaya, Ared, Kanyon and Chernorechenskaya, Krasnodar Region, as well as in several mines.

- Myotis davidii Peters, 1869. One genetically identified specimen of this species was collected in the Razvalka mine near Pyatigorsk, Stavropol Province. See Benda et al. (2016) for details.

- Myotis emarginatus (Geoffroy, 1806). Similar to the Greater Horseshoe Bat, the distribution of this species in the Caucasus is limited by the availability of suitable underground maternity sites. The species has not been found in the Eastern Caucasus and reported only twice from the central part of the region, including one record in a small cave near the Babugent settlement (Kabardino-Balkaria). Other 21 record locations lay in karstic areas of the Western Caucasus within the Kranodar Province and Adygea Republic. Maternity colonies were reported from the Khadzhokhskaya (now abandoned by bats), Dedova Yama, Teshebskaya, Svetlaya, Mordvinovskaya and Ared caves; hibernating individuals were recorded in the Ambitsukova, Fanagoriyskaya, Kabaniy Proval, Ared and Dedova Yama caves.

- Myotis mystacinus (Kuhl, 1817). The distribution of $M$. mystacinus in the region and its dependence on caves is unclear due to difficulties in a morphological identification of this species in its contemporary sense. Genetically identified specimens were collected in the Fanagoriyskaya Cave (Benda et al., 2016).
- Myotis nattereri (Kuhl, 1817). Recorded from 27 sites in the Russian Caucasus, this bat has rarely been found in caves and undergrounds. Few individuals were captured as they swarmed in the Akhunskaya, Pervomayskaya, Babailovskaya, Fanagoriyskaya and Kanyon caves, Krasnodar Province. In the latter two caves, single Natterer's bats were also found during winter. Nine pregnant females were netted on 4 June 2007 as they swarmed at the entrance to Kamennye Sarai Cave, Stavropol Province.

- Nyctalus noctula (Schreber, 1774). Roosting behaviour unusual for this species was first reported in 1980 for the Proval Cave, Stavropol Province (Gazaryan, Kazakov, 2002), in 2008 this colony remained in the cave. Only adult males used this cave in summer, whereas females were found there only between September and May.

- Pipistrellus pipistrellus (Schreber, 1774). A group of 30-40 hibernating pipistrelles was found in a deep crevice inside the Kamennye Sarai Cave, Stavropol Province on 20 December 2009. This is the only documented evidence of this species hibernating in Russian caves.

-Plecotus auritus (Linnaeus, 1758). Twenty-six of ca 60 records of this species in the region belong to underground sites, including 20 karst caves, where it was found both during the hibernation and swarming seasons. The largest number of the brown longeared bats netted at one roost did not exceed 15 animals, and only solitary individuals could usually be found in winter shelters. - Plecotus macrobullaris Kuzyakin, 1965. The sole record in an underground site was made in a mine at Razvalka Mountain, Stavropol Province on 21 December 2009. However, we anticipate future findings of this species in natural underground roosts. Family Miniopteridae Dobson, 1875 - Miniopterus schreibersii (Kuhl, 1817) (Fig. 7C). Among the Russian representatives of Chiroptera, Schreiber's Bent-wing bat shows the highest rate of dependence on caves and is very selective in terms of their 
size, accessibility and microclimate. Hence, it inhabits only the Western Caucasus with its vast karst areas, and is not distributed eastward to the Central and Eastern Caucasus; 34 record locations in the Adygea Republic, Krasnodar Province and Karachaevo-Cherkessia are available. All known records pertain to natural caves, with two exceptions when four individuals were observed in abandoned mines and one animal was found roosting under a bridge. The largest colonies (up to 5,000 bats) occupy spacious horizontal caves with large entrances, which make them exposed to disturbance by visitors and susceptible to vandalism and intentional killing. For these reasons, bats forsook some of the previously reported cave roosts and many colonies have depleted in numbers since the first extended survey (Gazaryan, Ostapenko, 1999). To avoid possible further decline and disturbance, we abstain from listing the names of these roosting sites.

Family Molossidae Gervais, 1856

- Tadarida teniotis (Rafinesque, 1814). This species does not inhabit caves in the strict sense of this term, but colonies could be found in ceiling crevices of rocky overhangs, where these bats may also swarm. Under such circumstances the free-tailed bats were found in a large grotto at the valley of the Cherek River in KabardinoBalkaria (Gazaryan, Tembotova, 2007).

\section{Conservation of caves and hy- pogean species}

Some of the karst cavities in European Russia are protected as objects of nature reserve stock, with various statuses allotted. Yet no dedicated animal life conservation is being performed in such caves. For example, the Shulgan-Tash (= Kapovaya) Cave in Bashkortostan (= Bashkiria), southern Cisuralia, is part of the Shulgan-Tash Federal State Nature Reserve as an object of the national historical and cultural legacy of the palaeolithic epoch. However, this very cave also hosts a few troglobiont spring- tails and mites, as well as a number of troglophiles amongst earthworms, spiders, beetles and flies (see above). In 1973, the Bolshaya Azishskaya and Fanagoriyskaya caves, both in the Krasnodar Province, were allotted the status of nature monuments. A good number of karst cavities are situated inside nature reserve stock objects of higher rank. Thus, numerous caves are located within the Sochi National Park or Caucasian Biosphere State Nature Reserve. Yet many caves like Shulgan-Tash, Bolshaya Azishskaya, Vorontsovskaya, Bolshaya Kazachebrodskaya etc., despite their protection statuses, are subjected to economic/excursion activities, this clearly rendering a negative effect on the biotas they host.

A number of cave animal species in European Russia are listed in regional red data books. Thus, the Red Data Book of the Moscow Region contains the amphipod crustacean Synurella ambulans (F. Müller, 1847). The Red Data Book of the Krasnodar Province lists several cave mollusks, crustaceans and many insects, while that of the Adygea Republic a few insects as well, all largely highly local endemic troglobionts (e.g. Turbanov et al. (2016a, b, c) and references therein). A number of bat species are likewise listed in several regional red data books. The main emphasis is thereby put on the conservation of caves as geological bodies, but not as shelters to the biotic components they support.

\section{Conclusion}

As a result, the subterranean biota of European Russia, including that of the northern Caucasus, but excluding that of Crimea, presently comprises at least 389 species or subspecies (some still unidentified) from 229 genera (a few still unidentified), 150 families, 75 orders, 48 classes and 25 phyla, among which 96 species or subspecies represent presumed stygo- or troglobionts. The taxonomically most diverse phylum is Arthropoda which encompasses the bulk of the fauna, in particular due to crustaceans, beetles and collembolans. Whereas the troglofauna of northern Russia is completely devoid of such strongly cave-adapted animals, in the 
Urals, Cis-Urals and central Russia there are a few, but the richest hypogean list in the region concerns the northern Caucasus, especially its western part where karst is particularly abundant.

\section{Acknowledgements}

The authors sincerely thank Yevgeny Zakharov (Sochi Branch of the Russian Geographical Society) for his very valuable comments on our work, including the polytoponymy of the caves of Greater Sochi, Krasnodar Province. Special thanks go to an anonymous referee who reviewed the submission and provided constructive criticism.

Field studies by Ilya Turbanov and Dmitry Palatov were performed in the framework of the state assignment of the Federal Agency for Scientific Organizations (FASO Russia) (topics Nos AAAA-A18-118012690106-7, AAAAA18-118012690105), supported in part also by the Russian Foundation for Basic Research (project No. 17-54-40017 Абx_a).

\section{References}

Abdullin S.R., Kapralov L.I., Kuzmina L.I., Ryss A.Y., Snit'ko V.P. et al. 2012. [The Biota of the ShulganTash (Kapovaya) Cave: A Popular-Science Essay]. Irgizly: Shulgan-Tash Nature Reserve. [In Russian]

Amirkhanov Z.M. 1980. [Distribution of bats in Dagestan] // Letuchie myshi (Chiroptera). [Bats (Chiroptera)]. Moscow: Nauka. P.63-69 [in Russian].

Andrejchuk V. 1996. Gypsum karst of the Pre-Ural Region, Russia // International Journal of Speleology. Vol.25. Nos 3-4. P.285-292.

Andrejchuk V.N., Klimchouk A.B. 1996. Gypsum karst of the Eastern-European Plain // International Journal of Speleology. Vol.25. Nos 3-4. P.251-261.

Antić D., Makarov S. 2016. The Caucasus as a major hotspot of biodiversity: Evidence from the millipede family Anthroleucosomatidae (Diplopoda, Chordeumatida) // Zootaxa. Vol.4211. No.1. P.1-205. https:// doi.org/10.11646/zootaxa.4211.1.1

Antić D.Ž., Turbanov I.S., Reboleira A.S.P.S. 2018. From the depths: Heterocaucaseuma deprofundum sp. nov., the world's deepest-occurring millipede (Diplopoda, Chordeumatida, Anthroleucosomatidae) from caves in the western Caucasus // Zootaxa. Vol.4377. No.1. P.110-124. https://doi.org/10.11646/zootaxa.4377.1.7

Babenko A.B. 1987. [Typhlogastrura (Collembola, Hypogastruridae) of the USSR] // Zoologicheskii zhurnal. Vol.66. No.3. P.463-472 [in Russian].

Babenko A.B. 2008. [Springtails (Collembola) of the karst landscapes of the Pinezhskiy Nature Reserve] // Zoo- logicheskii zhurnal. Vol.87. No.3. P.292-305 [in Russian].

Babenko A.B., Potapov M.B., Stebaeva S.K., Chernova N.M. 1994. [Collembola of Russia and adjacent countries: Family Hypogastruridae]. Moscow: Nauka. 336 pp. [In Russian]

Bakka A.I., Bakka S.V. 1999. [Bats of the Nizhniy Novgorod Region] // Plecotus et al. Vol.2. P.44-59 [in Russian].

Barjadze S., Murvanidze M., Arabuli T., Mumladze L., Pkhakadze V., Djanashvili R., Salakaia M. 2015. Annotated list of invertebrates of the Georgian karst caves. Tbilisi: Georgian Academic Book. 120 pp.

Behning A.L. 1928. [Some data on the fauna of wells in the city of Saratov] // Raboty Volzhskoy biologicheskoy stantsii. Vol.10. No.1. P.32-54 [in Russian].

Belousov I.A. 1989. [New ground beetles of the tribe Trechini (Coleoptera, Carabidae) from the Caucasus. II. New species of the genera Duvalius Delar and Nannotrechus Winkl., and a review of the grandiceps and caucasicus groups of the genus Trechus Clairv.] //Entomologicheskoe obozrenie. Vol.68. No.1.P.136153 [in Russian].

Belousov I.A. 1998. Le complexe générique de Nannotrechus Winkler du Caucase et de la Crimée (Coleoptera, Carabidae, Trechini). Sofia - Moscow - St. Petersburg: Pensoft Series Faunistica 8. 256 pp.

Belousov I.A. 1999. A new genus and species of cave dwelling beetles from the West Caucasus (Coleoptera, Carabidae, Trechini) // A. Zamotajlov, R. Sciaky (eds.). Advance in Carabidology; Papers dedicated to the memory of Prof. Oleg L. Kryzhanovskij. Krasnodar: Muiso. P.165-182.

Belousov I.A., Koval A.G. 2009. To the knowledge of the aphenopsoid trechine beetles (Coleoptera, Carabidae, Trechini) of the Caucasus // Caucasian Entomological Bulletin. Vol.5. No.2. P.163-173.

Belousov I.A., Koval A.G. 2011. A new cave-dwelling species of the genus Cimmerites Jeannel, 1928 (Coleoptera: Carabidae: Trechini) from the West Caucasus // Zoosystematica Rossica. Vol.20. No.1. P.48-53.

Belousov I., Zamotajlov A. 1997. A new blind genus of the tribe Trechini (Coleoptera, Carabidae) from the West Caucasus // Entomologica Basiliensia. Vol.20. P. 87100.

Belousov I.A., Zamotajlov A.S. 1999. A new Meganophthalmus species (Coleoptera, Carabidae, Trechini) from the west Caucasus // Entomologica Basiliensia. Vol.21. P.19-23.

Benda P., Gazaryan S., Vallo P. 2016. On the distribution and taxonomy of bats of the Myotis mystacinus morphogroup from the Caucasus region (Chiroptera: Vespertilionidae) // Turkish Journal of Zoology. Vol.40. http://doi:10.3906/zoo-1505-47

Besuchet C., Kurbatov S. 2007. Les Bryaxis du Caucase et du secteur oriental des chaînes Pontiques (Coleoptera: Staphylinidae: Pselaphinae) // Russian Entomological Journal. Vol.16. No.2. P.155-206.

Bey-Bienko G.Ya. 1965. On the cavernicolous grasshoppers of the genus Dolichopoda Bol. (Orthoptera) from the Caucasus $/ / 4^{\text {th }}$ International Congress of Speleol- 
ogy, Summaries of lectures. Ljubljana, Yugoslavia. P.73-74.

Bey-Bienko G.Ya. 1969. [Sur les Sauterelles cavernicoles du genre Dolichopoda Bol. (Orthoptera) en Caucasie] $/ /$ Actes du $4^{\text {th }}$ Congrès International de Spéléologie en Yougoslavie, Ljubljana. Nos 4-5. P.19-23 [in Russian].

Birstein Ya.A. 1948. [The occurrence of the cave shrimp Troglocaris in underground waters of Matsesta and related problems] // Byulleten Moskovskogo obshchestva ispytateley prirody, sect. Biol. Vol.53. No.1. P.3-10 [in Russian].

Birstein Ya.A. 1950. [Cave fauna of western Transcaucasia] // Zoologicheskii zhurnal. Vol.29. No.4. P.254366 [in Russian].

Birstein Ya.A. 1952. [Subterranean amphipods of the Khosta-Gudauta region (western Transcaucasia)] // Byulleten Moskovskogo obshchestva ispytatieley prirody, sect. Biol. Vol.57. No.1. P.26-39 [in Russian].

Birstein Ya.A. 1954. [A subterranean Niphargus amphipod (Crustacea, Amphipoda) recorded in the lower reaches of Don River and the Kuban basin] // Zoologicheskii zhurnal. Vol.33. No.5. P.1025-1031 [in Russian].

Birstein Ya.A. 1967. [Subterranean asellids (Crustacea, Isopoda) of Transcaucasia] // Zoologicheskii zhurnal. Vol.46. No.6. P.856-865 [in Russian].

Birstein Ya.A., Borutzky E.W. 1950. [Life in subterranean waters] // E.N. Pavlovsky, V.I. Zhadin (eds). Zhizn' presnykh vod SSSR. Tom 3. Glava 28. Moscow-Leningrad: Acad. Sci. USSR Publ. P.683-706 [in Russian].

Birstein Ya.A., Ljovuschkin S.I. 1960. [Biospeleological studies in western Transcaucasia in the autumn of 1959] // N.I. Sokolov (ed.). Materialy mezhduvedomstvennoy komissii po izucheniyu geologii i geografii karsta. [Digest of the Joint Commission for the Study Karst Geology and Geography], Issue 1. Moscow. P.174-177 [in Russian].

Birstein Ya.A., Ljovuschkin S.I. 1964. [A new subspecies of Bathynella natans Vejd. (Crustacea, Bathynellacea) from the subterranean waters of Ciscaucasia] // Zoologicheskii zhurnal. Vol.43. No.11. P.1719-1722 [in Russian].

Birstein Ya.A., Ljovuschkin S.I. 1965. [Subterranean Paraselloidea (Crustacea, Asellota) in the USSR] // Zoologicheskii zhurnal. Vol.44. No.7. P.997-1013 [in Russian].

Birstein Ya.A., Ljovuschkin S.I. 1966. [Subterranean fauna of the Russian Plain] // N.A Gvozdetski, A.G. Chikishev (eds). Voprosy izucheniya karsta Russkoy ravniny. [Problems of the Study of the Karst of the Russian Plain]. Moscow: Moskovskoe obshchestvo ispytateley prirody. P.115-120 [in Russian].

Birstein Ya.A., Ljovuschkin S.I. 1967a. [Some results and tasks of the study of the subterranean fauna of the USSR] // Zoologicheskii zhurnal. Vol.46. No.10. P.1509-1535 [in Russian].

Birstein Ya.A., Ljovuschkin S.I. 1967b. [Biospeologica Sovietica 33. The order Bathynellacea (Crustacea, Malacostraca) in the U.S.S.R. 1. The family Parabathynellidae] // Byulleten Moskovskogo obshchest- va ispytateley prirody, sect. Biol. Vol.72. No.4. P.5566 [in Russian].

Birstein Ya.A., Lopashov G. 1940. [Investigation of the cave fauna of the U.S.S.R. in 1935-1939] // Byulleten Moskovskogo obshchestva ispytateley prirody, sect. Biol. Vol.49. Nos 3-4. P.30-38. [in Russian].

Bol'shakov V.N., Orlov O.L., Snit'ko V.P. 2005. [Bats of the Urals] // Yekaterinburg: Akademkniga. 176 pp. [In Russian]

Borutzky E.W. 1926. [To the fauna of subterranean waters: Parastenocaris fonticola sp. n.] // Trudy Kosinskoy biologicheskoy stantsii. [Proceedings of the Kosinskaya Biological Station]. No.4. P.47-50 [in Russian].

Borutzky E.W. 1927. On the occurrence of the Amphipoda Synurella ambullans in Russia // Annals and Magazine of Natural History. Ser.9. Vol.20. P.63-66.

Borutzky E.W. 1930. Zur Kenntnis der unterirdischen Fauna der Kutais-Höhlen am Rion (Transkaukasus, Georgien). Copepoda - Cyclopoida // Zoologischer Anzeiger. Bd.89. S.121-132.

Borutzky E.W. 1934. Zur Harpacticoidenfauna des Kaukasus // Zoologischer Anzeiger. Bd.107. S.11-23.

Borutzky E.W. 1948a. [Copepoda Harpacticoida in caves of the Caucasus] // Byulleten Moskovskogo obshchestva ispytateley prirody, sect. Biol. Vol.53. No.4. P.3-12 [in Russian].

Borutzky E.W. 1948b. [Terrestrial Isopoda in caves of the Caucasus and Crimea. II. The family Trichoniscidae (subfamily Haplophthalminae)] // Vestnik Moskovskogo universiteta. Vol.5. P.137-146 [in Russian].

Borutzky E.W. 1950a. [Speocyclops lussianus sp. n. (Copepoda, Cyclopoida) en provenance des cavernes du Caucase] // Doklady Akademii nauk SSSR. Vol.71. No.4. P.777-779 [in Russian].

Borutzky E.W. 1950b. [Terrestrial Isopoda from caves of the Caucasus and Crimea. I. Fam. Ligiidae] // Byulleten Moskovskogo obshchestva ispytateley prirody, sect. Biol. Vol.55. No.5. P.69-81 [in Russian].

Borutzky E.W. 1952. [Harpaticoida of subterranean waters] // Fauna SSSR. Crustacea. Vol.3. No.4. MoscowLeningrad: USSR Acad. Sci. Publ. 425 pp. [In Russian]

Borutzky E.W. 1961. [New or little-known species of the genus Cylisticus (Isopoda Terrestria) of the USSR] // Sbornik trudov Zoologicheskogo muzeya MGU. No.8. P.29-46 [in Russian].

Borutzky E.W. 1965. [On the genus Speocyclops Kiefer (Copepoda, Cyclopoida)] // Zoologicheskii zhurnal. Vol.44. No.6. P.833-843 [in Russian].

Borutzky E.W. 1967a. [Biospeologica Sovietica 32. On the genus Nitocrella Chappuis (Copepoda, Harpacticoida) // Byulleten Moskovskogo obshchestva ispytateley prirody, sect. Biol. Vol.72. No.1. P.34-39 [in Russian].

Borutzky E.W. 1967b. [A new genus, Pseudobuddelundiella gen. n., and its position in the superfamily Trichoniscoidea (Isopoda terrestria)] // Zoologicheskii zhurnal. Vol.46. No.3. P.338-344 [in Russian].

Borutzky E.W. 1969c. [Psachonethes czerkessicus gen. et sp. n. (Isopoda terrestria) from caves in western Transcaucasia] // Zoologicheskii zhurnal. Vol.48. No.11. P.1618-1622 [in Russian]. 
Borutzky E.W. 1972a. [Isopoda Oniscoidea from caves of the Black Sea coast, Krasnodar Province] // Sbornik trudov Zoologicheskogo muzeya MGU. No.12. P.1936 [in Russian].

Borutzky E.W. 1972b. [Copepoda Harpacticoida from caves in western Transcaucasia] // Sbornik trudov Zoologicheskogo muzeya MGU. No.12. P.37-60 [in Russian].

Borutzky E.W., Mikhailova-Neikova M. 1970. [Copepoda Harpacticoida from caves of western Georgia, Azerbaijan and Middle Asia] // Zoologicheskii zhurnal. Vol.49. No.9. P.1334-1341 [in Russian].

Bronstein Z.S. 1947. [Ostracoda from freshwaters] // Fauna SSSR. Crustacea. Vol.2. No.1. Moscow-Leningrad: USSR Acad. Sci. Publ. 306 pp. [In Russian]

Charitonov D.E. 1947. [Spiders and harvestmen from caves of the Black Sea coast of the Caucasus] // Byulleten Moskovskogo obshchestva ispytateley prirody, sect. Biol. Vol.52. No.2. P.15-25 [in Russian].

Chemeris A.N. 2009. New data on the harvestman genus Nemaspela Šilhavy, 1966 (Arachnida, Opiliones) // Bulletin of the British Arachnological Society. Vol.14. Pt.7. P.286-296.

Chertoprud M.V. 2006. [Fauna and habitats of Amphipoda (Crustacea) in the Moscow Region] // Biologiya vnutrennikh vod. Vol.4. P.17-21 [in Russian].

Chertoprud E.S., Chertoprud M.V., Borisov R.R., Shavrina E.V. 2011. [Zoobenthos of near-Pinega cave systems, Arkhangelsk Region: faunistic diversity and ecological species complexes] // Materialy mezhdunarodnoy nauchnoy konferentsii "Karstovye sistemy Severa v menyayushcheysya srede". [Materials of the International Scientific Conference, "Karst systems of the North in a changing environment"]. Russia, Krasnaya Gorka, 5-10 September, 2011. P.109-113 [in Russian].

Chertoprud E.S., Palatov D.M., Borisov R.R., Marinskiy V.V., Bizin M.S., Dbar R.S. 2016. Distribution and a comparative analysis of the aquatic invertebrate fauna in caves of the western Caucasus // Subterranean Biology. Vol.18. P.49-70. http://doi.org/10.3897/subtbiol.18.8648

Chibisova O.I. 1967. [Testate amoebae (Testacea) from some cave and karst springs] // Zoologicheskii zhurnal. Vol.46. No.2. P.181-186 [in Russian].

Chikishev A.G. 1966. [Karst of the Kuloiskoe Plateau] // N.A. Gvodzdetzkii, A.G. Chikishev (eds.). Voprosy izucheniya karsta Russkoy ravniny. [Problems of the study of the karst of the Russian Plain]. Moscow: Moskovskoe obshchestvo ispytateley prirody. P.4052 [in Russian].

Chikishev A.G. 1973. [Caves in the territory of the USSR]. Moscow: Nauka. 136 pp. [In Russian]

Chikishev A.G. 1978. [Karst of the Russian Plain]. Moscow: Nauka. 192 pp. [In Russian]

Chikishev A.G. 1984. [Karst zonation in the USSR] // Zemlevedenie. Vol.15. No.55. P.56-60 [in Russian].

Dashdamirov S., Schawaller W. 1992. Pseudoscorpions of the Caucasian fauna (Arachnida Pseudoscorpiones) // Arthropoda Selecta. Vol.1. No.4. P.31-72.
Danielopol D. 1969. Notes sur la morphologie et la systématique de la sous famille Limnocytherinae Sars (Crustacea, Ostracoda) // Annales de Spéléologie. T.24. Fasc.2. P.129-142.

Davidian G.E. 2007. A new species of troglobiont weevil of the genus Otiorhynchus Germ. (Coleoptera, Curculionidae) from the western Caucasus // Caucasian Entomological Bulletin. Vol.3. No.2. P.193-195.

Derzhavin A.N. 1945. [The subterranean Amphipoda of Transcaucasia] // Byulleten Akademii nauk Azerbaijanskoy SSR. Vol.8. P.27-43 [in Russian, with English summary].

Dublyanskiy V.N., Ilyukhin V.V. 1982. [The largest karst caves and shafts in the USSR]. Moscow: Nauka. 137 pp. [In Russian]

Egorov R.V., Greke K. 2005. Orculoidea. Orculidae, Strobilopsidae // Treasure of Russian Shells. No.7. P.261.

Enghoff H. 1984. A revision of the Nopoiulinae, with notes on the classification of blaniulid millipedes (Diplopoda: Julida: Blaniulidae) // Senckenbergiana biologica. Bd.64. H.4/6. S.393-427.

Esyunin S.L., Efimik V.E. 1998. Remarks on the Urals spider fauna, 9. New data on the Ural species of the genus Lepthyphantes Menge, 1866 (s. 1.) (Aranei: Linyphiidae) // Arthropoda Selecta. Vol.7. No.3. P.227-232.

Evsyukov A.P., Golovatch S.I., Reip H.S. 2016. The millipede genus Strongylosoma Brandt, 1833 in the Caucasus (Diplopoda, Polydesmida, Paradoxosomatidae)// Acta Societatis Zoologicae Bohemicae. Vol.80. P.7-16.

Gazaryan S.V. 1999. [The Canyon cave - a unique hibernation site for bats] // Plecotus et al. Vol.2. P.126 [in Russian].

Gazaryan S.V. 2002. [Ecological and faunal analysis of bats (Chiroptera) of the Western Caucasus]. Dissertation ... of Candidate in Zoology. Moscow. [In Russian]

Gazaryan S.V., Kazakov B.A. 2002. [Ecology of the Noctule Bat in the North Caucasus. Communication 1: residential status, roosts, aggregation and mating behavior] // Plecotus et al. Pars specialis. P.74-82 [in Russian].

Gazaryan S.V. 2004. [The Pond Bat Myotis dasycneme (Boie, 1825) - a bat species new to the Caucasus] // Plecotus et al. Vol.7. P.102-103 [in Russian].

Gazaryan S.V. 2007a. Barbastella barbastellus // Krasnaya kniga Krasnodarskogo kraya. [Red Data Book of the Krasnodar Krai]. Krasnodar. P.421 [in Russian].

Gazaryan S.V. 2007b. [Distribution, biology, and conservation status of the Lesser Mouse-Eared Bat Myotis blythii in the Northern Caucasus] // Trudy Yuzhnogo nauchnogo tsentra Rossiyskoy akademii nauk. Rostov-na-Donu. [Studies of the Southern Scientific Centre of the Russian Academy of Sciences. Rostov-onDon]. P.284-299 [in Russian].

Gazaryan S.V. 2016. On new records of Rhinolophus euryale and incredibility of records of $R$. mehelyi in the Russian part of the Western Caucasus // Plecotus et al. Vol.19. P.41-50.

Gazaryan S.V., Tembotova F.A. 2007. [New records of bats in the Central Caucasus] // Zoologicheskii zhurnal. Vol.86. No.6. P.761-762 [in Russian]. 
Gazaryan S., Bukhnikashvili A., Kandaurov A., Natradze I. 2008. New status of Myotis daubentonii in the Caucasus // Abstracts of the XIth European Bat Research Symposium. Babeş-Bolyai University. P.50.

Gazaryan S., Ostapenko A. 1999. [Cave roosts of bats in the Western Caucasus which need protection] // Svet, Kiev. No.20. P.20-22 [in Russian].

Gazaryan S.V., Panyutina A.A. 2013. Barbastella barbastellus // Krasnaya kniga Karachaevo-Cherkesskoy Respubliki. [Red Data Book of the Karachay-Cherkess Republic]. Cherkessk. P.160 [in Russian].

Golovatch S.I. 1975. [Two genera of Oniscomorpha (Diplopoda) new to the USSR fauna, found in Transcaucasia, and their zoogeographical relations] // Zoologicheskii zhurnal. Vol.65. No.10. P.1566-1571 [in Russian].

Golovatch S.I. 1978. [New or little-known species of Diplopoda of the USSR European part] // Zoologicheskii zhurnal. Vol.57. No.3. P.453-457 [in Russian].

Golovatch S.I. 1983. Une nouvelle espèce cavernicole du genre Leucogeorgia Verh. de la Transcaucasie (Diplopoda, Julidae) // Fragmenta Entomologica. T.17. Fasc.1. P.47-50.

Golovatch S.I. 1984-1985. Two new genera of cavedwelling millipedes (Diplopoda) with remarks on the millipede fauna of west Caucasian caves // International Journal of Speleology. Vol.14. P.39-50.

Golovatch S.I. 1990. Diplopoda of the Caucasus, 3. Trachysphaeridae, with contributions to the fauna of Turkey // Senckenbergiana biologica. Bd.70. H.4/6. S.331358.

Golovatch S.I. 2011. The millipede genus Caucasodesmus Golovatch, 1985, with the description of a new species from the Crimea, Ukraine (Polydesmida, Diplopoda, Trichopolydesmidae) // ZooKeys. Vol.93. No.1. P.18. http://doi: 10.3897/zookeys.93.1159

Golovatch S.I., Chumachenko Y.A. 2013. The millipede Typhloglomeris caucasica Golovatch, 1975 found epigeically (Diplopoda, Glomerida, Glomeridellidae) // Biodiversity Data Journal. Vol.1. e981. http://doi: 10.3897/BDJ.1.e981

Golovatch S.I., Enghoff H. 1990. [The millipede, Nopoiulus kochii (Gervais, 1847) in the Caucasus (Diplopoda, Julida, Blaniulidae)] // B.R. Striganova (ed.). Fauna nazemnykh bespozvonochnykh Kavkaza. [Fauna of Terrestrial Invertebrates]. Moscow: Nauka. P.114-118 [in Russian].

Golovatch S.I., Evsyukov A.P., Reip H. 2016. The millipede family Polydesmidae in the Caucasus (Diplopoda: Polydesmida) // Zootaxa. Vol.4085. No.1. P.1-51. doi.org/10.11646/zootaxa.4085.1.1

Gorbunova K.A. 1965. [Peculiarities of gypsum karst]. Perm: Permskoe knizhnoe izdatel'stvo. 120 pp. [In Russian]

Gorbunova K.A. 1977. [Karst in gypsum of the USSR]. Perm: Perm University Press. 83 pp. [In Russian]

Gorbunova K.A. 1979. [Morphology and hydrogeology of gypsum karst]. Perm: Perm University Press. 95 pp. [In Russian]

Gorbunova K.A., Andrejchuk V.N., Kostarev V.P., Maksimovitch N.G. 1992. [Karst and caves in the Perm
Region]. Perm: Perm Univesity Press. 200 pp. [In Russian]

Gusarov V.I., Koval A.G. 2002. A revision of the genus Heinzia Korge, 1971 (Coleoptera: Staphylinidae: Quediina), with description of a new species and its probable larva // Zootaxa. Vol.69. P.1-19.

Gvozdetski N.A. 1965a. [Types of karst of the northern Caucasus] // Tipy karsta. Moscow: Nauka. P.47-56 [in Russian].

Gvozdetski N.A. 1965b. [Karst]. Moscow: Mysl'. 286 pp. [In Russian]

Hlaváč P., Kodada J., Koval A. 1999. A new cavernicolous species of Seracamaurops Winkler, 1925 (Coleoptera: Staphylinidae: Pselaphinae) from Caucasus // Revue suisse de Zoologie. T.106. Fasc.1. P.241-247.

Jie D., Potapov M., Sokolova M. 2011. Further study on the labial palp in the Isotomidae (Collembola) with reference to the genus Heteroisotoma Stach // Zootaxa. Vol.2991. P.49-61.

Juberthie C., Sidorov D., Decu V., Mikhaljova E.V., Semenchenko K. 2016. Subterranean fauna from Siberia and Russian Far East. Encyclopaedia Biospeologica (Siberia-Far East special issue) // Ecologica Montenegrina. Vol.7. P.507-529.

Kantor Y.I., Sysoev A.V. 2005. [Catalogue of the mollusks of Russia and adjacent lands]. Moscow: KMK Scientific Press. 627 pp. [In Russian]

Kantor Y.I., Vinarski M.V., Schileyko A.A., Sysoev A.V. 2010. Continental mollusks of Russia and adjacent territories. Internet version 2.3.1 - cited Dec. 2013. 12) Available: http://www. Ruthenica com/categorie8.html.

Kapralov S.A. 2015. [Arthropods in caves of the European part of Russia and the southern Urals] // Materialy vserossiyskoy molodezhnoy konferentsii "Biospeleologiya Kavkaza i drugikh rayonov Rossii”. [Materials of the All-Russia Youth Conference, "Biospeleology of the Caucasus and other regions of Russia"]. Kostroma: Kostromskoy Printing House. P.21-23 [in Russian].

Kapralov S.A., Chernorudskiy A.L. 2009. [Invertebrate populations in caves at Kamennoe, Nizhegorodskaya Region] // Peshchery: Sbornik nauchnykh rabot Permskogo gosudarstvennogo universiteta. [Caves: A collection of scientific papers of the Perm State University. Vol.32. P.94-99 [in Russian].

Kapterev P. 1910. Experimentelle Untersuchungen über die Frage den Einflusse der Dunkelheit auf die Gefühlsorgan der Daphnien-Augen // Biologisches Zentralblatt. Bd.30. S.239-256.

Kapterev P. 1912. Über den Einfluss der Dunkelheit auf die Daphnien-Augen // Biologisches Zentralblatt. Bd.32. S.233-243.

Karaman G. 1982. Contribution to the knowledge of the Amphipoda 101. Niphargus pseudocaspius n. sp. and $N$. caelestis, n. sp., new names for some Niphargus species (Fam. Gammaridae)// Poljoprivreda i Sumarstvo, Titograd. Vol.1. P.73-77.

Karaman G. 2012. New species of the subterranean genus Niphargus Schiödte, 1849 (Amphipoda, Gammaridea, 
Niphargidae) from Russia, N. krasnodarus sp. n. // Biologia Serbica. Vol.34. Nos 1-2. P.75-88.

Kazanbiev M.K. 1975. [Gypsum and salt karst in Dagestan and Checheno-Ingushetia] // Gidrogeologiya i karstovedenie. Perm. T.7. 197 pp. [In Russian]

Kiselyov V., Klimchouk A. 1991. Cave discoveries in the USSR (1990) // N.S.S. News, July. P.194-195.

Klimchouk A. 2003a. Caucasus, Georgia // J. Gunn (ed.). Encyclopedia of Cave and Karst Science. New York, London: Fitzroy Dearborn. P.200-203

Klimchouk A. 2003b. Russia, Ukraine // J. Gunn (ed.). Encyclopedia of Cave and Karst Science. New York, London: Fitzroy Dearborn. P.627-629.

Klimchouk A. 2003c. Soviet Union: speleological history // J. Gunn (ed.). Encyclopedia of Cave and Karst Science. New York, London: Fitzroy Dearborn. P.661663.

Klimchouk A., Forti P., Copper A. 1996. Gypsum karst of the World: a brief overview // International Journal of Speleology. Vol.25. Nos 3-4. P.159-181.

Kniss V.A. 1984a. [Sur la structure de la population de Plutomurus bashkiricus Skorikow, 1899, des cavernes de la Bachkirie du sud et de l'est] // [Fauna i ekologiya kollembol; La faune et l'écologie des Collemboles]. Moscow: Nauka. P.11-17 [in Russian].

Kniss V.A. 1984b. [Sur la faune des cavernes karstiques de la Bachkirie du sud et de l'est] // [Fauna i ekologiya kollembol; La faune et l'écologie des Collemboles]. Moscow: Nauka. P.123-128 [in Russian].

Kniss V.A. 1985. [Springtails of the genus Schaefferia (Collembola, Hypogastruridae) from caves in the southwest of Bashkiria] // Zoologicheskii zhurnal. Vol.64. No.6. P.934-937 [in Russian].

Kniss V.A. 1989. Sur la faune des cavernes de l'Oural du sud // Proceedings of the 10th International Congress of Speleology. Budapest. P.437-439.

Kniss V.A. 1991. Sur la faune cavernicole du Parc national de Baïkal (U.R.S.S.) // Mémoires de Biospéologie. T.18, 301-303.

Kniss V.A. 2001. [Cave fauna of Russia and adjacent regions]. Ufa: Bashkirian University Press. 238 pp. [In Russian]

Kniss V.A. 2004. [A zoogeographical analysis and the specificity of subterranean fauna in the former USSR] // Zoologicheskii zhurnal. Vol.83. No.5. P.615-620 [in Russian].

Kniss V.A. 2006. [Troglobiont springtails (Collembola, Apterygota) of the main karst regions of Russia] // Vestnik Bashkirskogo universiteta. Vol.1. P.63-68 [in Russian].

Kniss V.A., Smirnov A.I. 1990. The age of the ShulganTash Cave // Studia Carstologia (2). Proceedings of the International Conference on Anthropogenic Impact and Environmental Changes in Karst. Brno. P.45-48.

Kniss V.A., Thibaud J.-M. 1999. Le genre Plutomurus en Russie et en Géorgie (Collembola, Tomoceridae) // Revue française d'Entomologie. T.21. Fasc.2. P.57-64.

Komarov E.V. 1993. [A new species of ground beetle of the genus Meganophthalmus Kur. (Coleoptera: Carabidae) from Kabardino-Balkaria] // Entomologicheskoe obozrenie. Vol.72. No.2. P.363-367 [in Russian].

Koval A.G. 2004a. [To the study of the fauna of the Chortova Nora cave in the northwestern Caucasus] // Materialy nauchnoy konferentsii, posvyashchennoy 100-letiyu so dnya rozhdeniya S.M. YablokovaKhnzoriana. [Materials of the scientific conference dedicated to the $100^{\text {th }}$ birthday of S.M. IablokovKhnzorian]. Yerevan: Institute of Zoology. P.75-80 [in Russian].

Koval A.G. 2004b. [To the study of the Akhunskaya cave, northwestern Caucasus] // Peshchery: Mezhvuzovskii sbornik nauchnykh rabot. [Caves: An interdisciplinary collection of scientific papers]. Perm: Perm University Press. Vols 29-30. P.150-155 [in Russian].

Koz'minykh V.O. 2012. [Materials to the study of beetle diversity (Insecta, Coleoptera) of the natural monuments, "Ledyanaya Gora" and Kungurskaya Ice Cave, Perm Province] // Peshchery: Mezhvuzovskii sbornik nauchnykh rabot. [Caves: An interdisciplinary collection of scientific papers]. Perm: Perm University Press. Vol.35. P.67-75 [in Russian].

Krumpal M. 1984. Zwei neue Höhlen-Pseudoscorpionen aus der UdSSR (Pseudoscorpiones) // Biológia (Bratislava). Vol.39. P.637-646.

Kryzhanovskij O.L., Belousov A.I., Kabak I.I., Kataev B.B., Makarov V.K., Shilenkov V.G. 1995. A checklist of the ground-beetles of Russia and adjacent lands. Sofia - Moscow: Pensoft. 275 pp.

Kuzyakin A.P. 1950. [Bats: Systematics, way of life, and benefits for agriculture and forestry] // Moscow: Sovetskaya Nauka. 444 pp. [in Russian].

Lapshov I.I. 1940. [Cave Pseudoscorpiones of Transcaucasia] // Byulleten Moskovskogo obshchestva ispytateley prirody, sect. Biol. Vol.49. Nos 3-4. P.61-73 [in Russian].

Lepneva S.G. 1940. [Trichoptera from caves of Transcaucasia] // Byulleten Moskovskogo obshchestva ispytateley prirody, sect. Biol. Vol.49. Nos 3-4. P.79-86 [in Russian].

Ljovuschkin S.I. 1962. [To the cave fauna of the nearDniestr region] // Byulleten Moskovskogo obshchestva ispytateley prirody, sect. Biol. Vol.67. No.3. P.2937 [in Russian].

Ljovuschkin S.I. 1963. [Biospeleological incestigations in western Transcaucasia in the summer of 1960] // Novosti karstovedeniya i speleologii (Interdisciplinary commission for the study of karst geology and geography). Perm. Vol.3. P.66-68 [in Russian].

Ljovuschkin S.I. 1965. [A new species of troglobitic Caucasian Jeannelius (Coleoptera, Trechini) from the Anakopian cave near Novyi Afon] // Zoologicheskii zhurnal. Vol.44. No.8. P.1262-1265 [in Russian].

Ljovuschkin S.I. 1966. [Cave fauna of the main karstic regions of the USSR]. Thesis of Candidate (Ph.D.) of Biological Sci. Degree. Moscow University. 286 pp. [In Russian]

Ljovuschkin S.I. 1970. [New forms of Trechini (Coleoptera) from caves of western Transcaucasia] // Zoologicheskii zhurnal. Vol.49. No.11. P.1656-1672 [in Russian]. 
Ljovuschkin S.I. 1972a. Biospeologica sovietica. XLIX. Le premier représentant anophthalme des Trechini (Coléoptères Carabiques) de la Ciscaucasie // International Journal of Speleology. Vol.4. P.357-364.

Ljovuschkin S.I. 1972b. [On the interrelations between soil, interstitial and cave faunas] // M.S. Ghilarov (ed.). Problemy pochvennoy zoologii. Materialy IV vsesoyuznogo soveshchaniya, Baku, 1972. [Problems of Soil Zoology. Materials of the IV All-Union Conference, Baku, 1972]. Moscow: Nauka. P.92 [in Russian].

Ljovuschkin S.I. 1973. Les Trechinae cavernicoles (Coléoptères Carabiques) de l'URSS // Livre du Cinquantenaire de 1'Institut de Spéologie «Émile Racovitza». Bucureşti-Cluj: Ed. Acad. R. P. Romana. P.385-394.

Ljovuschkin S.I., Matiokin P.V. 1965. [Troglolestes sokolovi gen. n., sp. n., the first cave slug] // Byulleten Moskovskogo obshchestva ispytateley prirody, sect. Biol. Vol.70. No. 3. P.35-46. [in Russian].

Ljovuschkin S.I., Starobogatov Ya.I. 1963. [Biospeologia sovietica 48. Cave Opilionidea in Crimea and the Caucasus] // Byulleten Moskovskogo obshchestva ispytateley prirody, sect. Biol. Vol.68. No.1. P.41-51 [in Russian].

Lohmander H. 1936. Über die Diplopoden des Kaukasusgebietes // Göteborgs Kungliga Vetenskaps- och Vitterhets-Samhälles Handlingar. Följden 5. Ser. B. Bd.5. No.1. S.1-196.

Luchnik V.N. 1915. [On a new species of the genus Trechus from the Caucasus] // Entomologicheskiy vestnik. Vol.2. No.2. P.111-112 [in Russian].

Lysenin G.P. 1981. [Karst of the southeastern part of the Komi, USSR]// Gidrogeologiya i karstovedenie. Perm. P.40-47 [in Russian].

Makarova O.L. 2009. [Fauna of free-living gamasid mites (Parasitiformes, Mesostigmata) in the northern taiga: An analysis of zonal specificity] // Zoologicheskii zhurnal. Vol.88. No.9. P.1039-1054 [in Russian].

Makukhin V.A., Molodkin P.F. 1988. [Gypsum caves of the northern Caucasus] // Peshchery (Caves). Perm: Perm University Press. P.50-51 [in Russian].

Maksimovitch G.A. 1962. [Distribution and regionalization of karst of the USSR] // Gidrogeologiya i karstovedenie. Perm: Perm University Press. Vol.1. P.26 [in Russian].

Maksimovitch G.A. 1963. [The larst of Perm, 1]. Perm. 433 pp. [In Russian]

Maksimovitch G.A., Gorbunova K.A. 1958. [Karst of the Perm Region]. Perm. 183 pp. [In Russian]

Maksimovitch G.A., Kostarev V.P. 1973. [Karst regions of the Urals and Cisuralia] // Vosprosy fizicheskoy geografii Urala. Perm. Vol.1. P.166-177 [in Russian].

Malevich I.I. 1947. [Oligochaetes from caves of the Caucasus]// Byulleten Moskovskogo obshchestva ispytateley prirody, sect. Biol. Vol.52. No.4. P.1-12 [in Russian].

Malkov V.N., Gurkalo E.N. 1999. [Speleological regionalization and distribution of caves in the Arkhangelsk Region] // Peshchery: Mezhvuzovskii sbornik nauchnykh rabot. [Caves: An interdisciplinary collection of scientific papers]. Perm: Perm University Press. P.1015 [in Russian].

Malkov V.N., Nikolaev Y.I, Kuznetsova V.A. 1986. [Regionalization of the Pinega and Kuloi basins according to the settings and intensity of exogenous processes] // Geologiya i poleznye iskopaemye Arkhangelskoy oblasti. Moscow. P.154-174 [in Russian].

Malkov V.N., Nikolaev Y.I.., Luskan V.F. 1988. [Types of karst of the Pinezhye] // Peshchery v gipsakh i angidritakh. Perm: Perm University Press. P.46-50 [in Russian].

Marin I., Sokolova A. 2014. Redescription of the stygobitic shrimp, Troglocaris (Xyphocaridella) jusbaschjani Birstein, 1948 (Decapoda: Caridea: Atyidae) from Agura River, Sochi, Russia, with remarks on other representatives of the genus from Caucasus // Zootaxa. Vol.3754. No.3. P.277-298. doi: 10.11646/zootaxa.3754.3.3

Martens J. 2006. Weberknechte aus dem Kaukasus (Arachnida, Opiliones, Nemastomatidae) // Senckenbergiana biologica. Bd.86. H.2. S.145-210.

Martynov A.V. 1919. [On Malacostraca of the Rostov-onDon Area] // Protokol zasedaniya Obshchestva estestvoispytatelei pri Donskom universitete. Vol.1. No.3. P.39-53 [in Russian].

Martynov A.V. 1932. [To the knowledge of the fauna of the Black Sea coast of the Caucasus. I. Amphipoda] // Trudy Zoologicheskogo instituta AN SSSR. Vol.1. No.1. P.73-98 [in Russian].

Martynova E.F. 1969. [Tomoceridae (Collembola)] // Entomologicheskoe obozrenie. Vol.43. No.2. P.299314 [in Russian].

Mazei Y., Belyakova O., Trulova A., Guidolin L., Coppellotti O. 2012. Testate amoebae communities from caves of some territories in European Russia and NorthEastern Italy // Protistology. Vol.7. No.1. P.42-50.

Michaelsen W. 1910. Zur Kenntnis der Lumbriciden und ihrer Verbreitung // Ezhegodnik Zoologicheskogo muzeya Imperatorskoy akademii nauk. St. Petersburg. Vol.15. P.1-74.

Monchenko V.I. 1983. [Speocyclops cinctus sp.n. (Crustacea, Copepoda) from the northern slope of the Great Causasian Mountain Ridge] // Zoologicheskii zhurnal. Vol.62. No.5. P.681-688 [in Russian].

Monchenko V.I. 1984. [Cyclopids new to the fauna of the Soviet Union (Crustacea, Cyclopidae)] // Vestnik zoologii, Kiev. No.6. P.29-35 [in Russian].

Monchenko V.I. 1986. [A representative of the genus Speocyclops new to the fauna of the Soviet Union (Crustacea, Copepoda)] // Vestnik zoologii, Kiev. No.6. P.3-8 [in Russian].

Mukhanov A.V., Kapralov S.A. 2010. [Lepidopteran fauna (Lepidoptera) of caves in European Russia] // Vestnik Nizhegorodskogo universiteta. Vol.2. No.2. P.439-441 [in Russian].

Myl'nikov A.P., Tikhonenkov D.V. 2007. A new species of soil predatory flagellate, Colponema edaphicum sp. n., from Cave Vorontsovskaya, North Caucasus (Protista, Alveolata: Colponemidae) // Zoosystematica Rossica. Vol.16. No.1. P.1-4. 
Myl'nikov A.P., Tikhonenkov D.V., Ssimdyanov T.G. 2006. [Fauna and morphology of heterotrophic flagellates of cave waterbodies] // Zoologicheskii zhurnal. Vol.85. No.10. P.1164-1176 [in Russian].

Ognev I.F. 1910. Sur les modifications dans les organes des poissons rouges après un séjour de trois ans dans l'obscurité // Revue de Biologie. Vol.1. P.4.

Orlov O.L. 2000. [The fauna and ecology of bats (Mammalia, Chiroptera) in the Middle Urals]. Extended Abstract of Candidate of Science (Biology) Dissertation. Yekaterinburg. 16 pp. [In Russian]

Ostapenko A.A. 1993. [Caves of the Ekeptze-Gadyk Range] // Svet, Kiev. Vol.3. No.9. P.12-13. [in Russian].

Ostapenko A.A. 1994. [Continuous explorations on the Ekeptze-Gadyk Range, Nord-West Caucasus] // Svet, Kiev. Vols 1-3. P.11-13 [in Russian].

Palacios-Vargas J.G., Juberthie C., Reddell J. 2017. Mexico // Encyclopaedia Biospeologica. Vol. IIa, Mundos Subterráneos. Vols 25-26. P.1-101. http://www.umae. org/mundos/mundos2526.pdf

Palatov D.M., Sokolova A.M. 2015. [Amphibiotic insects in waters of caves in western Transcaucasia] // Materialy vserossiyskoy molodezhnoy konferentsii "Biospeleologiya Kavkaza i drugikh rayonov Rossii”. [Materials of the All-Russia Youth Conference, "Biospeleology of the Caucasus and other regions of Russia”]. Kostroma: Kostromskoy Printing House. P.5559 [in Russian].

Palatov D.M., Vinarski M.V. 2015. [Ecology and distribution of the gastropod subfamily Belgrandiellinae (Mollusca: Gastropoda: Hydrobiidae sensu lato) in the territory of the western Caucasus] // Materialy vserossiyskoy molodezhnoy konferentsii "Biospeleologiya Kavkaza i drugikh rayonov Rossii”. [Materials of the All-Russia Youth Conference, "Biospeleology of the Caucasus and other regions of Russia"]. Kostroma: Kostromskoy Printing House. P.49-54 [in Russian].

Pan'kov N.N. 2008a. [Invertebrates inhabiting caves of the Kungur region] // Peshchery: Sbornik nauchnykh rabot. [Caves: Collected scientific papers]. Perm: Perm State University. Vol.31. P.144-155 [in Russian].

Pan'kov N.N. 2008b. [The main results of studying Crangonyx chlebnikovi in the subterranean waters of the Kungur region] // Gornoe Ekho: Vestnik Gornogo instituta UrO RAN. Vol.1. No.31. P.29-40 [in Russian].

Pan'kov N.N., Andreeva A.I., Starova O.S., Maleev A.S., Shustov V.M. 2010. [On the physiology of the stygobiont amphipod Crangonyx chlebnikovi Borutzky, 1928 (Crangonyctidae)] // Peshchery: Sbornik nauchnykh rabot Permskogo gosudarstvennogo universiteta. [Caves: Collected Papers of the Perm State University. Perm: Perm University Press. Vol.33. P.136-142 [in Russian].

Pan'kov N.N., Andreeva A.I., Starova O.S., Maleev A.S., Shustov V.M. 2011. [Metabolism intensity and energy balance of the stygobiont amphipod Crangonyx chlebnikovi Borutzky, 1928 (Crangonyctidae)] // Vestnik Permskogo iniversiteta, seriya Biologiya. Vol.1. P.78-83 [in Russian].
Pan'kov N.N., Gorshkov N.G., Chernov A.V. 2005. [Crangonyx chlebnikovi (Amphipoda: Gammaridae) in the Kungur Ice Cave: Demography and reproductive biology] // Vestnik Permskogo iniversiteta, seriya Biologiya. Vol.6. P.77-82 [in Russian].

Pan'kov N.N., Pan'kova N.V. 2004. [On the biology of the troglobiont amphipod Crangonyx chlebnikovi Borutzky, 1928 (Gammaridae), with the description of a new subspecies from the Kungur Ice Cave] // Peshchery: Sbornik nauchnykh rabot Permskogo gosudarstvennogo universiteta. [Caves: Collected Papers of the Perm State University]. Perm: Perm University Press. Vol.29-30. P.141-150 [in Russian].

Pan'kov N.N., Starova O.S. 2009a. [On the population biology of Crangonyx chlebnikovi Borutzky, 1928 (Amphipoda: Gammaridae) from three caves of the Cis-Ural region] // Peshchery Peshchery: Sbornik nauchnykh rabot Permskogo gosudarstvennogo universiteta. [Caves: A collection of scientific papers]. Perm: Perm University Press. Vol.32. P.84-93 [in Russian].

Pan'kov N.N., Starova O.S., Pan'kova N.V. 2009b. [Invertebrate animals in caves of the Perm Province: fauna, ecological structure, chorology and seasonal dynamics] // Peshchery: Sbornik nauchnykh rabot Permskogo gosudarstvennogo universiteta. [Caves): A collection of scientific papers]. Perm: Perm University Press. Vol.32. P.72-83 [in Russian].

Pan'kov N.N., Starova O.S. 2009c. [Demography and reproductive biology of Crangonyx chlebnikovi Borutzky, 1928 (Amphipoda: Gammaridae) in three caves of the Cis-Ural region] // Vestnik Permskogo universiteta, seriya Biologiya. Vol.10. P.55-61 [in Russian].

Perkovsky E.E. 1991. [To the leiodid beetle fauna (Coleoptera, Leiodidae) of caves in the Soviet Union] // Byulleten Moskovskogo obshchestva ispytateley prirody, sect. Biol. Vol.96. No.1. P.71-73 [in Russian].

Pichka V.E. 1965. [On the spider fauna of caves in western Transcaucasia] // Zoologicheskii zhurnal. Vol.44. No.8. P.1190-1196 [in Russian].

Potapov M.B. 1991. Species of the genus Isotoma, subgen. Parisitoma Bagnall, 1840 and Serisotoma subgen. nov. (Collembolla, Isotomidae) of USSR fauna // Acta Zoologica Cracoviensia. Vol.34. No.1. P.267-301.

Read H.J. 1992. The genus Cylindroiulus Verhoeff, 1894 in the faunas of the Caucasus, Turkey and Iran (Myriapoda: Diplopoda: Julidae) // Senckenbergiana biologica. Bd.72. Nos 4-6. S.373-433.

Reisner V., Shelepin A. 1997. Exploration of the "KrestikTurist" cave system // Proceedings of the $12^{\text {th }}$ International Congress of Speleolology, Switzerland, Symposium 4. P.103-106.

Riedel A. 1966. Zonitidae (excl. Daudebardiinae) der Kaukasusländer (Gastropoda) // Annales Zoologici. Vol.24. No.1. P.1-303.

Reitter E. 1888-1889. Coleopteren aus Circassien, gesammelt von Hans Leder im Jahre 1887 // Wiener entomologische Zeitung. Bd.7. S.19-26, 81-90, 143-156, 169-180, 207-214, 231-238, 259, 274, 279-288, $317-321$. 
Rudjakov I.A. 1963. [On cave ostracods of Transcaucasia] // Zoologicheskii zhurnal. Vol.42. No.1. P.28-31 [in Russian].

Rykov A.M. 2014. [Flying echo-locators] // Zapovednyi Kray, Pinega. Vol.56. No.4. P.3 [in Russian].

Salomon J.N., Pulina M. 2005. Le karst et les phénomènes karstiques similaires des régions froides // J.N. Salomon, M. Pulina (eds.). Les karsts des régions climatiques extrêmes. Karstologia. Mémoire 14. P.11100. Bibliographie. P.195-204.

Satunin K.A. 1912. [Excursions to caves in the Sukhum region. The giant caves Ablaskira and Aszaba] // Izvestiya Kavkazskogo otdela Russkogo geograficheskogo obshchestva. Vol.21. No.1. P.13-18 [in Russian].

Schileyko À.À. 1988. [New or little-known species of land snails (Pulmonata) in the USSR fauna, based on material from the Zoological Museum of the Moscow University] // Zoologicheskii zhurnal. Vol.67. No.9. P.1303-1313 [in Russian].

Schütt H., Şeşen R. 1993. Pseudamnicola species and other freshwater gastropods (Mollusca, Gastropoda) from East Anatolia (Turkey), the Ukraine and the Lebanon // Basteria. Vol.57. P.161-171.

Semenov A.P. 1901. [The Caucasian cave representative of the genus Dolichopoda Bol.] // Russkoe entomologicheskoe obozrenie. Vol.1. P.5-9 [in Russian].

Semenov-Tian-Shanskiy A.P. 1909. [A new representative of cave fauna in the Caucasus, Laemostenus (Pristonychus) tschitscherini, sp. n. (Coleoptera, Carabidae)] // Russkoe entomologicheskoe obozrenie. Vol.8. Nos 3-4. P.312-313 [in Russian].

Semikolennykh A.A. 1997. Microorganisms in the caves of the former SSSR: geography, ecology and geochemical activity // Proceedings of the $12^{\text {th }}$ Internatuibal Congress of Speleolology, Switzerland. Vol.3. P.293299.

Serban E. 1993. Tianschanobathynella jankowskajae n. g. sp. n. et Tianschanobathynella paraissykhulensis n. sp. (Bathynellidae, Bathynellacea, Podophallocarida) // Travaux de l'Institut de Spéologie «Émile Racovitza». T.32. P.19-41.

Shapovalov M.I., Zamotajlov A.S., Bondarenko A.S. 2015. New provincial records of the cave cricket, Dolichopoda euxina Semenov, 1901 (Orthoptera) from the Republic of Adygea (Russia) with notes on its conservation status // Caucasian Entomological Bulletin. Vol.11. No.2. P.267-269.

Schäfer A.W. 1951. Ueber die Besiedlung des Grundwasser // Verhandlungen des Internationalen Vereins für Limnologie. Bd.11. S.324-330.

Shugurov A.M. 1912. [Materials to the study of the geographical distribution of orthopterans in Taurida Governorate] // Zapiski Novorossiyskogo Imperatorskogo obshchestva estestvoispytateley, Odessa. Vol.37. P.145-167 [in Russian].

Shumeev A.N. 2008. [Freshwater and land planarians of the northwestern Caucasus]. Thesis of Candidate (Ph.D.) of Biological Sci. Degree. St. Petersburg. 144 pp. [In Russian]
Sidorov D.A. 2015. The spring-dwelling amphipod genus Lyurella (Peracarida, Amphipoda): Systematics, distribution, and affinity, with description of the second representative from the Black Sea coast region // Crustaceana. Vol.88. No.1. P.27-50.

Sidorov D.A., Chertoprud E.S., Semikolennykh A.A., Shavrina E.B. 2011. [An enigmatic Pallasea (Crustacea: Amphipoda) from the Golubinskiy Proval and Kitezh caves] // Materialy mezhdunarodnoy nauchnoy konferentsii "Karstovye sistemy Severa v menyayushcheysya srede". [Materials of the International Scientific Conference, "Karst systems of the North in a changing environment"]. Russia, Krasnaya Gorka, 5-10 September 2011. P.85-87 [in Russian].

Sidorov D.A., Pan'kov N.N., Holsinger J.R. 2012. Distribution and ecology of the subterranean amphipod Crangonyx chlebnikovi Borutzky, 1928 (Crustacea: Crangonyctidae), with lectotype designation and comments on morphology of the lateral cephalic lobe // Arthropoda Selecta. Vol.21. No.2. P.149-160.

Sidorov D.A., Pan'kov N.N, Krasheninnikov A.B. 2010. A Bactrurus-like subterranean amphipod (Crangonyctidae) from the Ural mountain karst region // A. Moškrić, P. Trontelj (eds.). Abstracts of the 20th International Conference on Subterranean Biology. Postojna. P.45.

Sidorov D.A., Palatov D. 2012. Taxonomy of the springdwelling amphipod Synurella ambulans (Crustacea: Crangonictidae) in West Russia // European Journal of Taxonomy. Vol.23.P.1-15.https://doi.org/10.5852/ ejt.2012.23

Skorikow A.S. 1899. Eine neue Tomocerus-Art (Collembola) aus Ost-Russland//Ezhegodnik Zoologicheskogo muzeya Imperatorskoy akademii nauk. St. Petersburg. Vol.4. P.473-480.

Smirnov D.G. 2001. [Records of bats in the Akhstyr cave (Krasnodar Province)] // Plecotus et al. Vol.4. P.6468 [in Russian].

Snit'ko V.P. 2004. [The fauna of bats (Mammalia, Chiroptera) of the southern Urals]. Autoreferate of the Thesis of Candidate (Ph.D.) of Biological Sci. Degree. Yekaterinburg. 25 pp. [In Russian]

Snit'ko V.P. 2007. [Seasonal spatial differentiation of sex groups in populations of resident bat species (Chiroptera, Vespertilionidae) in the southern Urals] // Russian Journal of Ecology. Vol.38. No.5. P.334-340 [in Russian].

Snit'ko V.P. 2009. [Records of bats (Chiroptera, Vespertilionidae) in hiberbation and during the period of activity in the vicinities of Sludorudnik settlement (Chelyabinsk Oblast)] // Ekologiya v vysshey shkole: Sintez nauki i obrazovaniya. Trudy vserossiyskoy konferentsii. [Ecology at the high school: a synthesis of science and education, Proceedings of the All-Russian conference]. Chelyabinsk. P.109-115 [in Russian].

Snit'ko V.P. 2011. [Natterer's bat Myotis nattereri (Kuhl, 1817) at the eastern range limit (the Urals)] // Zoologicheskii zhurnal. Vol.90. No.10. P.1245-1254 [in Russian]. 
Snit'ko V.P., Snit'ko L.V. 2016. Bats (Chiroptera, Vespertillionidae) from the Cis-Urals and South Urals (Republic of Bashkortostan) // Biology Bulletin. Vol.43. No.7. P.707-728.

Snit'ko V.P., Snit'ko L.V. 2017a. [New data on the distribution of bats (Chiroptera, Vespertillionidae) in the Orenburg Region] // Zoologicheskii zhurnal. Vol.96. No.1. P.83-89 [in Russian].

Snit'ko V.P., Snit'ko L.V. 2017b. [Bats (Chiroptera, Vespertellionidae) of the southern Urals (Chelyabinsk Region)] // Zoologicheskii zhurnal. Vol.96. No.3. P.320-349 [in Russian].

Sokolova A.M., Palatov D.M. 2015. [Stygobiont fauna of the Black Sea coast in the environs of Tuapse] // Materialy vserossiyskoy molodezhnoy konferentsii "Biospeleologiya Kavkaza i drugikh rayonov Rossii". [Materials of the All-Russia Youth Conference, "Biospeleology of the Caucasus and other regions of Russia"]. Kostroma: Kostromskoy Printing House. P.7679 [in Russian].

Spassky S.A. 1932. Aranearum species novae // Bulletin du Muséum d'histoire naturelle, Paris. Série 2. T.4. Fasc.2. P.182-189; Fasc.8. P.972-979.

Spassky S.A. 1934. Araneae Palaearcticae novae. Fam. Pholcidae 1 // Bulletin du Muséum national d'Histoire naturelle, Paris. Sér.2. T.6. P.361-372.

Starobogatov Y.I. 1962. [Contribution aux mollusques des eaux souterraines du Caucase] // Byulleten Moskovskogo obshchestva ispytateley prirody, sect. Biol. Vol.67. No.6. P.42-54 [in Russian].

Strasser K. 1970. Über einige Diplopoden aus dem westlichen Kaukasus // Revue suisse de Zoologie. T.77. Fasc.1. P.199-205.

Strelkov P.P. 1958. [Materials on winter roosts of bats in the European Soviet Union] // Trudy Zoologicheskogo instituta AN SSSR. Leningrad. Vol.25. P.255-303 [in Russian].

Strelkov P.P. 1990. [From the first to fifth All-Union conferences on bats] // Materialy 5-oi vsesoyuznoy konferentsii po letuchim mysham. [Proceedings of the $5^{\text {th }}$ All-Union Bat Conference]. Penza. P.4-7 [in Russian].

Stupishin A.V. 1965. [Karst of the Volga region] // Tipy karsta v SSSR. Moscow: Nauka. [In Russian].

Sukhovey L.N. 1992. [New data on gypsum caves of the Northern Caucasus] // Svet, Kiev. Vol.4. No.6. P.1213 [in Russian].

Torsuev N.P. 1975. [Distribution of gypsum karst on the Timansky Ridge] // Gidrogeologia i karstovedenie. Perm: Perm University Press. Vol.7. P.74-75 [in Russian].

Tsytsulina E.A. 1999. New records of bats (Chiroptera) in the Western Caucasus // Plecotus et al. Vol.2. P.79-83.

Turbanov I.S., Marin I.N. 2015. [Typhlogammaridae, a family of stygobiont amphipods (Crustacea) new to the fauna of Russia, found in the subterranean waters of Krasnodar Province] // Materialy vserossiyskoy molodezhnoy konferentsii "Biospeleologiya Kavkaza i drugikh rayonov Rossii". [Materials of the AllRussia Youth Conference, "Biospeleology of the Cau- casus and other regions of Russia"]. Kostroma: Kostromskoy Printing House. P.87-89 [in Russian].

Turbanov I.S., Marin I.N. 2017. The record of the stygobiotic amphipod family Typhlogammaridae (Crustacea) from the Avgust Cave at Sochi, Krasnodar Region, as new to the Russian fauna // Ukrainian Journal of Ecology. Vol.7. No.4. P.465-468.

Turbanov I.S., Nadolny A.A. 2017. [Some data on the species composition of caves spiders (Aranei) of the western Caucasus] // Materialy II vserossiyskoy molodezhnoy konferentsii "Biospeleologicheskie issledovaniya v Rossii i sosednikh stranakh". [Materials of the II All-Russia Youth Conference, "Biospeleological research in Russia and neighboring countries"]. Yaroslavl: Filigran. P.113-117 [in Russian, English summary].

Turbanov I.S., Palatov D.M., Golovatch S.I. 2016a. [The state of the art of biospeleology in Russia and other countries of the former Soviet Union: A review of the cave (endogean) invertebrate fauna. I. Introduction Crustacea] // Zoologicheskii zhurnal. Vol.95. No.10. P.1136-1159 [in Russian; English translation: Entomological Review, 2016, Vol.96. No.7. P.926-963].

Turbanov I.S., Palatov D.M., Golovatch S.I. 2016b. [The state of the art of biospeleology in Russia and other countries of the former Soviet Union: A review of the cave (endogean) invertebrate fauna. 2. Arachnida Acknowledgements]//Zoologicheskii zhurnal. Vol.95. No.11. P.1283-1304 [in Russian; English translation: Entomological Review, 2016. Vol.96. No.9. P.12971333].

Turbanov I.S., Palatov D.M., Golovatch S.I. 2016c. [The state of the art of biospeleology in Russia and other countries of the former Soviet Union: A review of the cave (endogean) invertebrate fauna. 3. Bibliography and summary] // Zoologicheskii zhurnal. Vol.95. No.12. P.1399-1426 [in Russian; English translation: Entomological Review, 2016. Vol.96. No.9. P.12971333].

Vereshchagina T.N. 1985. [The carabid genus Laemostenus Bon. (Coleoptera, Carabidae) in the Caucasus. 1. Species of the L. koenigi Rtt. group] // Entomologicheskoe obozrenie. Vol.64. No.3. P.554-562 [in Russian].

Vereshchagina T.N., Makarov K.V. 1986. [The carabid genus Laemostenus Bon. (Coleoptera, Carabidae) in the Caucasus. II. Larvae of the L. koenigi Rtt. group] //Entomologicheskoe obozrenie. Vol.65. No.2.P.367373 [in Russian].

Verhoeff K.W. 1930. Eine neue cavernicole Symphyognathen-Gattung aus Georgien, Transcaucasien // Mitteilungen über Höhlen- und Karstforschung. H.2. S.3339.

Vinarski M.V., Kantor Y.I. 2016. Analytical catalogue of fresh and brackish water molluscs of Russia and adjacent countries. Moscow: Severtsov Institute of Ecology and Evolution, Russian Academy of Sciences. 544 pp.

Volkova Y.S. 2015. [Application of DNA barcoding to the species identification of troglobiont lithobiomorphs 
(Chilopoda, Lithobiomorpha)] // Materialy vserossiyskoy molodezhnoy konferentsii "Biospeleologiya Kavkaza i drugikh rayonov Rossii”. [Materials of the All-Russia Youth Conference, "Biospeleology of the Caucasus and other regions of Russia"]. Kostroma: Kostromskoy Printing House. P.13-17 [in Russian]. Volodin A.P., Pshenichnikov V.A. 1949. [Microflora of the Kungur Ice Cave] // Proceedings of the Perm Speleological Institute. Vol.8. P.393-402 [in Russian].

Waltham T 1994. Gypsum caves of Pinega // International Caver. Vol.10. P.15-22.

Waltham T. 2003. Pinega gypsum caves, Russia // J. Gunn (ed.). Encyclopedia of Cave and Karst Science. New York, London: Fitzroy Dearborn. P.588-589.

Waltham T., Cooper A. 1988. Features of gypsum caves and karst at Pinega (Russia) and Ripon (England) // Cave and Karst Science. Vol.25. P.131-140.

Winkler A. 1926. Eine neue Gattung blinder Trechinen aus dem Kaukasus // Koleopterologische Rundschau. Bd.12. H.3/4. S.168-171.
Zaitsev F.A. 1948. [Review of the cave fauna of Georgia] // Trudy Instituta zoologii Gruzinskoy SSR. Vol.8. P.151-173 [in Russian].

Zalesskaja N.T. 1963. [On chilopod Myriapoda of Crimea and the Caucasus] // Zoologicheskii zhurnal. Vol.42. No.7. P.1022-1030 [in Russian].

Zalesskaja N.T. 1973a. [Lithobiidae (Chilopoda) of the Caucasus: Their distribution and association with soils] // M.S. Ghilarov (ed.). Ekologiya pochvennykh bespozvonochnykh. [Ecology of Soil Invertebrates]. Moscow: Nauka. P.120-130 [in Russian].

Zalesskaja N.T. 1973b. [A new cave-dwelling form (Lithobiidae, Chilopoda) from the Caucasus (Biospeologica Sovietica LI)]// Zoologicheskii zhurnal. Vol.52. No.1. P.136-138 [in Russian].

Zalesskaja N.T. 1978. [Identification book of lithobiomorph centipedes of the USSR]. Moscow: Nauka. 212 pp. [In Russian]

Responsible editor K.G. Mikhailov 